\title{
Existence Theorems in Linear Chaos
}

\section{Stanislav Shkarin*}

Queens's University Belfast, Pure Mathematics Research Centre, University Road, Belfast, UK

\begin{abstract}
Chaotic linear dynamics deals primarily with various topological ergodic properties of semigroups of continuous linear operators acting on a topological vector space. In this survey paper, we treat questions of characterizing which of the spaces from a given class support a semigroup of prescribed shape satisfying a given topological ergodic property.

In particular, we characterize countable inductive limits of separable Banach spaces that admit a hypercyclic operator, show that there is a non-mixing hypercyclic operator on a separable infinite dimensional complex Fréchet space $X$ if and only if $X$ is non-isomorphic to the space $\omega$ of all sequences with coordinatewise convergence topology. It is also shown for any $k \in \mathbb{N}$, any separable infinite dimensional Fréchet space $X$ non-isomorphic to $\omega$ admits a mixing uniformly continuous group $\left\{T_{t}\right\}_{t \in C^{n}}$ of continuous linear operators and that there is no supercyclic strongly continuous operator semigroup $\left\{T_{t}\right\}_{\geq 00}$ on $\omega$. We specify a wide class of Fréchet spaces $X$, including all infinite dimensional Banach spaces with separable dual, such that there is a hypercyclic operator $T$ on $X$ for which the dual operator $T^{\prime}$ is also hypercyclic. An extension of the Salas theorem on hypercyclicity of a perturbation of the identity by adding a backward weighted shift is presented and its various applications are outlined.
\end{abstract}

Keywords: Hypercyclic operators; Mixing semigroups; Backward weighted shifts; Bilateral weighted shifts

\section{Introduction}

Unless stated otherwise, all vector spaces in this article are over the field $\mathbb{K}$, being either the field $\mathbb{C}$ of complex numbers or the field $\mathbb{R}$ of real numbers, all topological spaces are assumed to be Hausdorff and all vector spaces are assumed to be non-trivial. As usual, $\mathbb{T}=\{z \in \mathbb{C}:|z|=1\}$, $\mathbb{Z}$ is the set of integers, $\mathbb{Z}$ is the set of non-negative integers, $\mathbb{N}$ is the set of positive integers and $\mathbb{R}_{+}$is the set of non-negative real numbers. Symbol $L(X, Y)$ stands for the space of continuous linear operators from a topological vector space $X$ to a topological vector space $Y$. We write $L(X)$ instead of $L(X, X)$ and $X^{\prime}$ instead of $L(X, \mathbb{K})$. For each $T \in$ $L(X)$, the dual operator $T^{\prime}: X^{\prime} \rightarrow X^{\prime}$ is defined as usual: $\left(T^{\prime} f\right)(x)=f(T x)$ for $f \in X^{\prime}$ and $x \in X$. By a quotient of a topological vector space $X$ we mean the space $X / Y$, where $Y$ is a closed linear subspace of $X$. We start by recalling some definitions and facts.

\section{Notation and definitions}

A topological vector space is called locally convex if it has a base of neighborhoods of zero consisting of convex sets. Equivalently, a topological vector space is locally convex if its topology can be defined by a family of seminorms. For brevity, we say locally convex space for a locally convex topological vector space. A subset $B$ of a topological vector space $X$ is called bounded if for any neighborhood $U$ of zero in $X$, a scalar multiple of $U$ contains $B$. We say that $\tau$ is a locally convex topology on a vector space $X$ if $(X, \tau)$ is a locally convex space. If $X$ is a vector space and $Y$ is a linear space of linear functionals on $X$ separating points of $X$, then the weakest topology on $X$, with respect to which all functionals from $Y$ are continuous, is denoted $\sigma(X, Y)$. The elements of $X$ can be naturally interpreted as linear functionals on $Y$, which allows one to consider the topology $\sigma(Y, X)$ as well. If $\mathcal{B}$ is a family of bounded subsets of $(Y, \sigma(Y, X))$, whose union is $Y$, then the seminorms

$$
p_{B}(x)=\sup _{f \in B}|f(x)| \text { for } B \in \mathcal{B}
$$

define the topology on $X$ of uniform convergence on sets of the family $\mathcal{B}$. The topology on $X$ of uniform convergence on all bounded subsets of $(Y, \sigma(Y, X))$ is called the strong topology and denoted $\beta(X, Y)$. The topology of uniform convergence on all compact convex subsets of $(Y$, $\sigma(Y, X))$ is called the Mackey topology and is denoted $\tau(X, Y)$. According to the Mackey-Arens theorem, for a locally convex space $(X, \tau)$ and a space $Y$ of linear functionals on $X$, the equality $Y=X^{\prime}$ holds if and only if $\sigma(X, Y) \subseteq \tau \subseteq \tau(X, Y)$. We say that a locally convex space $(X, \tau)$ carries a weak topology if $\tau$ coincides with $\sigma(X, Y)$ for some space $Y$ of linear functionals on $X$, separating points of $X$. If $X$ is a locally convex space, we write $X_{\beta}$ for $\left(X^{\prime}, \beta\left(X, X^{\prime}\right)\right), X_{\tau}$ for $\left(X, \tau\left(X, X^{\prime}\right)\right)$ and $X_{\sigma}$ for $(X, \sigma(X$, $\left.X^{\prime}\right)$ ). Similarly we denote $\left(X, \beta\left(X^{\prime}, X\right)\right)$ by $X_{\beta}{ }^{\prime},\left(X^{\prime}, \tau\left(X^{\prime}, X\right)\right)$ by $X_{\tau}{ }^{\prime}$ and $\left(X^{\prime}, \sigma\left(X^{\prime}, X\right)\right)$ by $X_{\sigma}^{\prime}$. An $\mathcal{F}$-space is a complete metrizable topological vector space. A locally convex $\mathcal{F}$-space is called a Fréchet space. If $\left\{X_{\alpha}\right.$ : $\alpha \in A\}$ is a family of locally convex spaces, then their (locally convex) direct sum is the algebraic direct sum $X=\bigoplus_{\alpha \in A} X_{\alpha}$ of the vector spaces $X_{\alpha}$ endowed with the strongest locally convex topology, which induces the original topology on each $X_{\alpha}$. Let $\left\{X_{n}\right\}_{n \in \mathbb{Z}_{+}}$be a sequence of vector spaces such that $X_{n}$ is a subspace of $X_{n+1}$ for each $n \in Z_{+}$and each $X_{n}$ carries its own locally convex topology $\tau_{n}$ such that $\tau_{n}$ is (maybe nonstrictly) stronger than the topology $\left.\tau_{\mathrm{n}+1}\right|_{X n} ^{n}$. Then the inductive limit of the sequence $\left\{X_{n}\right\}$ is the space $X=\bigcup_{n=0}^{\infty} X_{n}$ endowed with the strongest locally convex topology $\tau$ such that $\left.\tau\right|_{X n} \subseteq \tau_{n}$ for each $n \in \mathbb{Z}_{+}$. In other words, a convex set $U$ is a neighborhood of zero in $X$ if and only if $U$ $\cap X_{n}$ is a neighborhood of zero in $X_{n}$ for each $n \in \mathbb{Z}_{+}$. An LB-space is an inductive limit of a sequence of Banach spaces. An $L B_{-}$-space is an inductive limit of a sequence of separable Banach spaces. We use

*Corresponding author: Stanislav Shkarin, Queens's University Belfast, Pure Mathematics Research Centre, University Road, Belfast, BT7 1NN, UK, Tel: +442890245133; E-mail: s.shkarin@qub.ac.uk

Received July 21, 2015; Accepted August 03, 2015; Published August 31, 2015

Citation: Shkarin S (2015) Existence Theorems in Linear Chaos. J Generalized Lie Theory Appl S1: 009. doi:10.4172/1736-4337.S1-009

Copyright: ( 2015 Shkarin S. This is an open-access article distributed under the terms of the Creative Commons Attribution License, which permits unrestricted use, distribution, and reproduction in any medium, provided the original author and source are credited. 
symbol $\varphi \varphi$ to denote the locally convex direct sum of countably many copies of the one- dimensional space $\mathbb{K}$ and the symbol $\omega$ to denote the product of countably many copies of $\mathbb{K}$. Note that $\varphi$ is a space of countable algebraic dimension and carries the strongest locally convex topology (=any seminorm on $\varphi$ is continuous). We can naturally interpret $\omega$ as the space $\mathbb{K}^{\mathbb{N}}$ of all sequences with coordinatewise convergence topology. Clearly $\omega$ is a separable Fréchet space. Recall also that if $X$ is a locally convex space and $A \subset X^{\prime}$, then $A$ is called uniformly equicontinuous if there exists a neighborhood $U$ of zero in $X$ such that $|f(\mathrm{x})| \leq 1$ for any $x \in U$ and $f \in A$.

Let $T$ be a continuous linear operator on a topological vector space $X$. A vector $x \in X$ is called a cyclic vector for $T$ if the linear span of the orbit $O(T, x)=\left\{T^{n}: n \in \mathbb{Z}\right\}$ of $x$ is dense in $X$. The operator $T$ is called cyclic if $T$ has a cyclic vector. Recall also that for $n \in \mathbb{N}, T$ is called $n$-cyclic if there are vectors $x_{1}, \ldots, x_{n} \in X$ such that the linear span of the set $\left\{T^{n} x_{j}: n \in \mathbb{Z}, 1 \leq j \leq n\right\}$ is dense in $X$. Obviously, 1-cyclicity coincides with cyclicity. We say that $T$ is multicyclic if it is $n$-cyclic for some $n \in \mathbb{N}$.

Let $X$ and $Y$ be topological spaces and $\left\{T_{a}: a \in A\right\}$ be a family of continuous maps from $X$ to $Y$. An element $x \in X$ is called universal for this family if the orbit $\left\{T_{a} x: a \in A\right\}$ is dense in $Y$ and $\left\{T_{a}: a \in A\right\}$ is said to be universal if it has a universal element. We say that a family $\left\{T_{n}: n \in\right.$ $\left.\mathbb{Z}_{+}\right\}$is hereditarily universal if any its infinite subfamily is universal. An operator semigroup on a topological vector space $X$ is a family $\left\{T_{t}\right\}_{t} \in_{A}$ of elements of $L(X)$ labeled by elements of an abelian monoid $A$ (monoid is a semigroup with identity) and satisfying $T_{0}=I, T_{s+t}=T_{t} T_{s}$ for any $t, s \in$ $A$ (unless stated otherwise, we use additive notation for the operation on $A$ ). A norm on $A$ is a function $|\cdot|: A \rightarrow[0, \infty)$ satisfying $|n a|=n|a|$ and $|a+b| \leq|a|+|b|$ for any for any $n \in \mathbb{Z}$ and $a, b \in A$. An abelian monoid equipped with a norm will be called a normed semigroup. We will be mainly concerned with the case when $A$ is a closed (additive) subsemigroup of $\mathbb{R}^{k}$ containing 0 with the norm $|a|$ being the Euclidean distance from $a$ to 0 . In the latter case we consider $A$ to be equipped with topology inherited from $\mathbb{R}^{k}$ and we say that an operator semigroup $\left\{T_{t}\right\}_{t \in A}$ is strongly continuous if the map $t \mapsto T_{t} x$ from $A$ to $X$ is continuous for any $x \in X$. We say that an operator semigroup $\left\{T_{t}\right\}_{t \in A}$ is uniformly continuous if there exists a neighborhood $U$ of zero in $X$ such that for any sequence $\left\{t_{n}\right\}_{n \in \mathbb{Z}_{+}}$of elements of $A$ converging to $t \in A, T_{t n} x$ converges to $T x$ uniformly on $U$. Clearly, uniform continuity is strictly stronger than strong continuity. It is also worth mentioning that many authors use the term 'uniformly continuous semigroup' for semigroups satisfying the weaker of uniform convergence of $T_{t n} x$ to $T_{t} x$ on any bounded subset of $X$.

If $A$ is a normed semigroup and $\left\{T_{t}\right\}_{t \in A}$ is an operator semigroup on a topological vector space $X$, then we say that $\left\{T_{t}\right\}_{t \in A}$ is (topologically) transitive if for any non-empty open subsets $U, V$ of $X$, the set $\{|t|: t \in A$, $\left.T_{t}(U) \cap V \neq \emptyset\right\}$ is unbounded. We say that $\left\{T_{t}\right\}_{t \in A}$ is (topologically) mixing if for any non-empty open subsets $U, V$ of $X$, there is $r=r(U, V)>0$ such that $T_{t}(U) \cap V \neq \varnothing$ provided $|t|>r$. We also say that $\left\{T_{t}\right\}_{t \in A}$ is hypercyclic (respectively, supercyclic) if the family $\left\{T_{t}: t \in A\right\}$ (respectively, $\left\{z T_{t}\right.$ : $z \in \mathbb{K}, t \in A\}$ ) is universal. $\left\{T_{t}\right\}_{t \in A}$ is said to be hereditarily hypercyclic (respectively, hereditarily supercyclic) if for any sequence $\left\{t_{n}\right\}_{n \in \mathbb{Z}_{+}}$of elements of $A$ such that $\left|t_{n}\right| \rightarrow \infty$, the family $\left\{T_{t n}: n \in \mathbb{Z}_{+}\right\}$(respectively, $\left.\left\{z T_{t n}: z \in \mathbb{K}, n \in \mathbb{Z}_{+}\right\}\right)$is universal. A continuous linear operator $T$ acting on a topological vector space $X$ is called hypercyclic, supercyclic, hereditarily hypercyclic, hereditarily supercyclic, mixing or transitive if the semigroup $\left\{T^{n}\right\}_{n \in \mathbb{Z}}$ has the same property. It is worth noting that our definition of a hereditarily hypercyclic operator follows Ansari [1], while in the terminology of references [2,3], the same property is called 'hereditarily hypercyclic with respect to the sequence $n_{k}=k$ of all nonnegative integers'. Hyper- cyclic and supercyclic operators have been intensely studied during last few decades, [4-6] and references therein. Clearly mixing implies transitivity and hereditary hypercyclicity (respectively, hereditary supercyclicity) implies hypercyclicity (respectively, supercyclicity). Recall that a topological space $X$ is called a Baire space if the intersection of countably many dense open subsets of $X$ is dense in $X$. According to the classical Baire theorem, complete metric spaces are Baire.

Proposition 1.1. Let $X$ be a topological vector space, $A$ be a normed semigroup and $S=\left\{T_{a}\right\}_{a \in A}$ be an operator semigroup on $X$. Then

\section{(1.1.1) if $\mathcal{S}$ is hereditarily hypercyclic, then $\mathcal{S}$ is mixing}

If additionally $X$ is Baire separable and metrizable, the converse implication holds:

\section{(1.1.2) if $\mathcal{S}$ is mixing, then $\mathcal{S}$ is hereditarily hypercyclic.}

The above proposition is a combination of well-known facts, appearing in the literature in various modifications. It is worth noting that a similar statement holds for hypercyclicity and transitivity under certain natural additional assumptions. One can also write down and prove a supercyclicity analogue of the above proposition. In the next section we shall prove Proposition 1.1 for sake of completeness. It is worth noting that for any subsemigroup $A_{0}$ of $A$, not lying in the kernel of the norm, $\left\{T_{t}\right\}_{t \in A_{0}}$ is mixing if $\left\{T_{t}\right\}_{t \in A}$ is mixing. In particular, if $\left\{T_{t}\right\}_{t \in A}$ is mixing, then $T_{t}$ is mixing whenever $|\mathrm{t}|>0$.

\section{Results}

The question of existence of supercyclic or hypercyclic operators or semigroups on various types of topological vector spaces was intensely studied. There are no hypercyclic operators on any finite dimensional topological vector space and there are no supercyclic operators on a finite dimensional topological vector space of real dimension $>2$. These facts follow, for instance from the main result of reference [7]. Herzog [8] demonstrated that there is a supercyclic operator on any separable infinite dimensional Banach space. Later Ansari [9] and Bernal-Gonzáles [10], answering a question raised by Herrero, showed independently that for any separable infinite dimensional Banach space $X$ there is a hypercyclic operator $T \in L(X)$. Using the same idea as in reference [9], Bonet and Peris [11] proved that there is a hypercyclic operator on any separable infinite dimensional Fréchet space and demonstrated that there is a hypercyclic operator on an inductive limit $X$ of a sequence $X$ for $n \in \mathbb{Z}$ of separable Banach spaces provided there is $n \in \mathbb{Z}_{+}$for which $X_{n}$ is dense in $X$. Grivaux [3] observed that hypercyclic operators $T$ constructed in references [9-11] are in fact mixing and therefore hereditarily hypercyclic. They actually come from the same source. Namely, according to Salas [12] an operator of the shape $I+T$, where $T$ is a backward weighted shift on $\ell_{1}$, is hypercyclic. Virtually the same proof as in reference [12] demonstrates that these operators are in fact mixing. Moreover, all operators constructed in the above papers, except for the ones acting on $\omega$, are hypercyclic because of a quasisimilarity with one of the operators of the shape identity plus a backward weighted shift. The same quasisimilarity transfers the mixing property as effectively as it transfers hypercyclicity. A similar idea was used by Bermúdez, Bonilla and Martinón [13] and BernalGonzález and Grosse-Erdmann [14], who have demonstrated that any separable infinite dimensional Banach space supports a hypercyclic strongly continuous semigroup $\left\{T_{t}\right\}_{t \in R+}$. Bermúdez, Bonilla, Conejero and Peris [15] have shown for any separable infinite dimensional complex Banach space $X$, there exists a mixing strongly continuous 
semigroup $\left\{T_{t}\right\}_{t \in \Pi}$ with $\Pi=\{z \in \mathbb{C}: \operatorname{Re} z \geq 0\}$ such that the map $t \mapsto T_{t}$ is holomorphic on the interior of $\Pi$. As a matter of fact, one can easily see that the semigroup constructed in reference [15] extends to a holomorphic mixing group $\left\{T_{t}\right\}_{t \in \mathbb{C}^{C}}$. Finally, Conejero [16] proved that any separable infinite dimensional complex Fréchet space nonisomorphic to $\omega$ supports a uniformly continuous mixing operator semigroup $\left\{T_{t}\right\}_{t \in R+}$.

The following theorem extracts the maximum of the method both in terms of the class of spaces and semigroups. Although the general idea remains the same, the proof requires dealing with a number of technical details of various nature. In particular, we will prove and apply a multi- operator version of the Salas theorem. For brevity we shall introduce the following class of locally convex spaces.

Definition 1.2. We say that a sequence $\left\{x_{n}\right\}_{n \in \mathbb{Z}_{+}}$of elements of a topological vector space $X$ is an $\ell_{1}$-sequence if $x_{n} \rightarrow 0$ in $X$ and the series $\sum_{n=0}^{\infty} a_{n} x_{n}$ converges in $X$ for each $a \in \ell_{1}$.

We say that a locally convex space $X$ belongs to the class $\mathfrak{M}$ if its topology is not weak and there exists an $\ell_{1}$-sequence in $X$ with dense span.

Theorem 1.3. Let $X \in \mathfrak{M}$. Then for any $k \in \mathbb{N}$, there exists a hereditarily hypercyclic (and therefore mixing) uniformly continuous operator group $\left\{T_{t}\right\}_{t \in \mathbb{K}^{\mathrm{k}}}$. Moreover, if $\mathbb{K}=\mathbb{C}$ the map $z \mapsto T_{z} X$ from $\mathbb{C}^{\mathrm{k}}$ to $X$ is holomorphic for each $x \in X$.

Since for any hereditarily hypercyclic semigroup $\left\{T_{t}\right\}_{t \in \mathbb{K k}}$ and any non-zero $t \in \mathbb{K}^{k}$, the operator $T_{t}$ is hereditarily hypercyclic, we have the following corollary.

Corollary 1.4. Let $X \in \mathfrak{M}$. Then there is a hereditarily hypercyclic (and therefore mixing) operator $T \in L(X)$.

Remark 1.5. It is easy to see that if $X \in \mathfrak{M}$, then $X_{\tau} \in \mathfrak{M}$. Indeed, if $\left\{x_{n}\right\}_{n \in \mathbb{Z}_{+}}$is an $\ell_{1}$-sequence in $X$ with dense span, then $\left\{2^{-n} x_{n}\right\}_{n \in \mathbb{Z}_{+}}$is an $\ell_{1}$-sequence in $X_{\tau}$ with dense span. Of course, $X_{\sigma}$ never belongs to $\mathfrak{M}$. On the other hand, it is well-known that $L\left(X_{\sigma}\right)=L\left(X_{\tau}\right)$. Moreover, since $\sigma\left(X, X^{\prime}\right) \subseteq \tau\left(X, X^{\prime}\right)$, then any strongly continuous hereditarily hypercyclic operator semigroup $\left\{T_{t}\right\}_{t \in \mathbb{K}_{\mathrm{k}}}$ on $X_{\tau}$ is also strongly continuous and hereditarily hypercyclic as an operator semigroup on $X_{\sigma}$. Thus in the case $X_{\tau} \in \mathfrak{M}$, Theorem 1.3 implies that there is a strongly continuous hereditarily hypercyclic operator semigroup $\left\{T_{t}\right\}_{t \in \mathbb{K} k}$ on $X_{\sigma}$. Unfortunately, the nature of the weak topology does not allow to make such a semigroup uniformly continuous.

It is worth noting that any separable Fréchet space admits an $\ell_{1}$ sequence with dense span. It is also well-known [17] that any Fréchet space carries the Mackey topology and the topology on a Fréchet space $X$ differs from the weak topology if and only if $X$ is infinite dimensional and is non-isomorphic to $\omega$. That is, any separable infinite dimensional Fréchet space non-isomorphic to $\omega$ belongs to $\mathfrak{M}$. Similarly, one can verify that an infinite dimensional inductive limit $X$ of a sequence $X_{n}$ for $n \in \mathbb{Z}_{+}$of separable Banach spaces belongs to $\mathfrak{M}$ provided there is $n$ $\in \mathbb{Z}_{+}$for which $X_{n}$ is dense in $X$. Thus all the above mentioned existence theorems are particular cases of Theorem 1.3.

Grivaux [3] raised a question whether each separable infinite dimensional Banach space supports a hypercyclic non-mixing operator. Since the class $\mathfrak{M}$ contains separable infinite dimensional Banach spaces, the following theorem provides an affirmative answer to this question.
Theorem 1.6. Let $X \in \mathfrak{M}$. Then there exists $T \in L(X)$ such that $T$ is hypercyclic and non-mixing.

The simplest separable infinite dimensional locally convex space space (and the only Fréchet space) outside $\mathfrak{M}$ is $\omega$. Curiously, the situation with $\omega$ is totally different. Hypercyclic operators on the complex space $\omega$ have been characterized by Herzog and Lemmert [18]. Namely, they proved that a continuous linear operator $T$ on the complex Fréchet space $\omega$ is hypercyclic if and only if the point spectrum $\sigma_{p}\left(T^{\prime}\right)$ of $T^{\prime}$ is empty. It also worth mentioning that Bés and Conejero [19] provided sufficient conditions for $T \in L(\omega)$ to have an infinite dimensional closed linear subspace, each non-zero vector of which is hypercyclic, and found common hypercyclic vectors for some families of hypercyclic operators on $\omega$. See also the related work [20] by Petersson. The following theorem extends the result of Herzog and Lemmert and highlights the difference between $\omega$ and other Fréchet spaces.

Theorem 1.7. Let $T \in L(\omega)$ be such that $T^{\prime}$ has no non-trivial finite dimensional invariant subspaces and $\{P l\}_{l \in \mathbb{Z}+}$ be a sequence of polynomials such that $\operatorname{deg} p_{l} \rightarrow \infty$ as $l \rightarrow \infty$. Then the family $\left\{p_{l}(T): l \in\right.$ $\left.\mathbb{Z}_{+}\right\}$is universal. Moreover, there is no strongly continuous supercyclic semigroup $\left\{T_{t}\right\}_{t \in \mathbb{R}_{+}}$on $\omega$.

Note that in the case $\mathbb{K}=\mathbb{C}, T^{\prime}$ has no non-trivial finite dimensional invariant subspaces if and only if $\sigma_{p}\left(T^{\prime}\right)=\emptyset$. The first part of the above theorem implies that any hypercyclic operator on $\omega$ is mixing. We shall, in fact, verify the following more general statement.

Theorem 1.8. Let $X$ be a locally convex space carrying weak topology and $T \in L(X)$. Then the following conditions are equivalent

(1.8.1) T' has no non-trivial finite dimensional invariant subspaces;

(1.8.2) $T$ is transitive;

(1.8.3) $T$ is mixing;

(1.8.4) the semigroup $\{p(T)\}_{p \in \mathcal{P}_{*}}$ is mixing, where $\mathcal{P}^{*}=\mathbb{K}[z] \backslash\{0\}$ is the multiplicative semigroup of non-zero polynomials with the norm $|p|=\operatorname{deg} p$.

Remark 1.9. Chan and Sanders [21] observed that on the space $\left(\ell_{2}\right)_{\sigma}$, being the Hilbert space $\ell_{2}$ with the weak topology, there is a transitive non-hypercyclic operator. Theorem 1.8 provides a huge supply of such operators. For instance, the backward shift $T$ on $\ell_{2}$ is mixing on $\left(\ell_{2}\right) \sigma\left(T^{\prime}\right.$ has no non-trivial finite dimensional invariant subspaces) and $T$ is clearly non-hypercyclic (each its orbit is bounded).

Theorems 1.3, 1.6 and 1.7 imply the following curious corollary.

Corollary 1.10. Let $X$ be a separable infinite dimensional Fréchet space. Then the following are equivalent

\section{(1.10.1) there is a hypercyclic non-mixing operator $T \in L(X)$;}

(1.10.2) there is a mixing uniformly continuous semigroup $\left\{T_{t}\right\}_{t \in \mathbb{R}_{+}}$ on $X$;

(1.10.3) there is a supercyclic strongly continuous semigroup $\left\{T_{t}\right\}_{t \in \mathbb{R}+}$ on $X$;

\section{(1.10.4) $X$ is non-isomorphic to $\omega$.}

Another simple space outside $\mathfrak{M}$ is $\varphi$. Bonet and Peris [11] observed that there are no supercyclic operators on $\varphi$. On the other hand, Bonet, Frerick, Peris and Wengenroth [22] constructed a hypercyclic operator on the locally convex direct sum $x=\bigoplus_{n=0}^{\infty} \ell_{1}$ of countably many 
copies of the Banach space $\ell_{1}$. The space $X$ is clearly an $\mathrm{LB}_{\mathrm{s}}$-space, is complete and non-metrizable. It is also easy to see that $X \notin \mathfrak{M}$ (there are no $\ell_{1}$-sequences in $X$ with dense span). We find sufficient conditions of existence and of non-existence of a hypercyclic operator on a locally convex space. These conditions allow us to characterize the LBs-spaces, which admit a hypercyclic operator.

Theorem 1.11. Let $X$ be the inductive limit of a sequence $\left\{X_{n}\right\}_{n \in \mathbb{Z}+}$ of separable Banach spaces. Then the following conditions are equivalent:

(1.11.1) X admits no hypercyclic operator;

(1.11.2) $X$ admits no cyclic operator with dense range;

(1.11.3) $X$ is isomorphic to $Y \times \varphi$, where $Y$ is the inductive limit of a sequence $\left\{Y_{n}\right\}_{n \in \mathbb{N}}$ of separable

Banach spaces such that $Y_{0}$ is dense in $Y$;

(1.11.4) for any sufficiently large $n, \bar{X}_{n+1} / \bar{X}_{n}$ is finite dimensional and the set $\left\{n \in \mathbb{Z}_{+}: \bar{X}_{n+1} \neq \bar{X}_{n}\right\}$ is infinite, where $\bar{X}_{k}$ is the closure of $X_{k}$ in $X$.

The proof is based upon the following result, which is of independent interest.

Theorem 1.12. Let $X$ be a topological vector space, which has no quotients isomorphic to $\varphi$. Then there is no cyclic operator with dense range on $X \times \varphi$.

The following theorem provides another generalization of the mentioned result of Bonet, Frerick, Peris and Wengenroth.

Theorem 1.13. Let $\left\{X_{n}\right\}_{n \in \mathbb{Z}_{+}}$be a sequence of separable Fréchet spaces. Then there is a hypercyclic operator on $X=\bigoplus_{n=0}^{\infty} X_{n}$ if and only if the set $\left\{n \in \mathbb{Z}_{+}: X_{n}\right.$ is infinite dimensional $\}$ is infinite.

We derive the above theorem from the following result, concerning more general spaces.

Theorem 1.14. Let $X_{n} \in \mathfrak{M}$ for each $n \in \mathbb{Z}_{+}$and $X=\bigoplus_{n=0}^{\infty} X_{n}$. Then there is a hypercyclic operator on $X$.

The next issue, we discuss, are dual hypercyclic operators. Let $X$ be a locally convex space. Recall that $X_{\beta}^{\prime}$ is the dual space $X^{\prime}$ endowed with the strong topology $\beta\left(X^{\prime}, X\right)$. It is worth noting that if $X$ is a normed space, then the strong topology on $X^{\prime}$ coincides with the standard norm topology. Salas [23] has constructed an example of a hypercyclic operator $T$ on $\ell_{2}$ such that both $T$ and $T^{\prime}$ are hypercyclic. This result motivated Petersson [24] to introduce the following definition. We say that a continuous linear operator $T$ on a locally convex space $X$ is dual hypercyclic if both $T$ and $T^{\prime}$ are hypercyclic on $X$ and $X_{\beta}{ }^{\prime}$ respectively. Using the construction of Salas, Petersson proved that any infinite dimensional Banach space $X$ with a monotonic and symmetric Schauder basis and with separable dual admits a dual hypercyclic operator. He also raised the following questions. Does there exist a dual hypercyclic operator on any infinite dimensional Banach space with separable dual? Does there exist a non-normable Fréchet space that admits a dual hypercyclic operator? The first of these questions was recently answered affirmatively by Salas [25]. The following theorem provides a sufficient condition for existence of a dual hypercyclic operator on a locally convex space.

Theorem 1.15. Let $X$ be an infinite dimensional locally convex space admitting an $\ell_{1}$-sequence with dense span. Assume also that there is an $\ell_{1}$-sequence $\left\{f_{n}\right\}_{n \in \mathbb{Z}_{+}}$with dense span in $X_{\beta}^{\prime}$ and at least one of the following conditions is satisfied:
(1.15.1) the topology of $X$ coincides with $\sigma\left(X, X^{\prime}\right)$;

(1.15.2) the topology of $X$ coincides with $\tau\left(X, X^{\prime}\right)$;

(1.15.3) the set $\left\{f_{n}: n \in \mathbb{Z}_{+}\right\}$is uniformly equicontinuous.

Then $X$ admits a dual hypercyclic operator.

Since every separable Fréchet space admits an $\ell_{1}$-sequence with dense span and every Fréchet space carries the Mackey topology, the above theorem implies the following corollary.

Corollary 1.16. Let $X$ be a separable infinite dimensional Fréchet space, such that there is an $\ell_{1}$-sequence with dense span in $X_{\beta}{ }^{\prime}$. Then there exists a dual hypercyclic operator $T \in L(X)$.

If $X$ is a Banach space, $X_{\beta}{ }^{\prime}$ is also a Banach space and therefore has an $\ell_{1}$-sequence with dense span if and only if it is separable. Thus Corollary 1.16 implies the next corollary, which is the mentioned recent result of Salas.

Corollary 1.17. Let $X$ be an infinite dimensional Banach space with separable dual. Then there exists a dual hypercyclic operator $T \in L(X)$.

Corollary 1.16 also provides plenty of non-normable Fréchet spaces admitting a dual hypercyclic operator, thus answering the second of the above questions of Petersson. For instance, take the complex Fr' echet space $X$ of entire functions on one variable with the topology of uniform convergence on compact sets. It is easy to verify that the sequence of functionals $g_{n}(f)=(n !)^{-1} f^{n)}(0)$ is an $\ell_{1}$-sequence with dense span in $X_{\beta}{ }^{\prime}$. Since $X$ is also infinite dimensional and separable, Corollary 1.16 implies that $X$ supports a dual hypercyclic operator.

The proofs of the above results are based upon the two main ingredients. One of them are sufficient conditions of mixing and the other is a criterion for a generic (in the Baire category sense) operator from a given class to be hypercyclic. Our sufficient conditions of mixing extend the result of Salas on hypercyclicity of perturbations of the identity by adding a backward weighted shift. Apart from providing us with tools, these extensions are of independent interest.

Theorem 1.18. Let $X$ be a topological vector space and $T \in L(X)$ be such that the space

$$
\Lambda(T)=\operatorname{span}\left(\bigcup_{n \in \mathbb{N},|z|=1}\left((T-z I)^{n}(X) \cap \operatorname{ker}(T-z I)^{n}\right)\right)
$$

is dense in $X$. Then T is mixing. If additionally, $X$ is Baire, separable and metrizable, then $T$ is hereditarily hypercyclic.

We shall see that the above theorem implies not only the mentioned result of Salas, but also is applicable in many other situations. For instance, we use the above theorem to prove the following results.

Theorem 1.19. Let $X$ be a separable infinite dimensional Banach space and $\mathcal{N}$ be the operator norm closure in $L(X)$ of the set of finite rank nilpotent operators. Then the set of $T \in \mathcal{N}$ for which $T$ is supercyclic and $I+T$ is hypercyclic is a dense $G_{\delta}$ subset of the complete metric space $\mathcal{N}$. If additionally $X^{\prime}$ is separable, then the set of $T \in \mathcal{N}$ for which $T$ and $T^{\prime}$ are supercyclic and $I+T$ and $I+T^{\prime}$ are hypercyclic is a dense $G_{\delta}$ subset of $\mathcal{N}$.

Note that if a Banach space $X$ has the approximation property [26], then the set $\mathcal{N}$ from the above corollary is exactly the set of compact quasinilpotent operators (in the case $\mathbb{K}=\mathbb{R}$ by quasinilpotency of $T$ we mean quasinilpotency of the complexification of $T$ or equivalently that $\|\left. T^{n}\right|^{1 / n} \rightarrow 0$ ). Thus we have the following corollary. 
Corollary 1.20. Let $X$ be a separable infinite dimensional Banach space with the approximation property and $\mathcal{N} \subset L(X)$ be the set of compact quasinilpotent operators. Then the set of $T \in \mathcal{N}$ for which $T$ is supercyclic and $I+T$ is hypercyclic is a dense $G_{\delta}$ subset of the complete metric space $\mathcal{N}$. If additionally $X^{\prime}$ is separable, then the set of $T \in \mathcal{N}$ for which $T$ and $T^{\prime}$ are supercyclic and $I+T$ and $I+T^{\prime}$ are hypercyclic is a dense $G_{\delta}$ subset of $\mathcal{N}$.

Theorem 1.21. Let $X$ be a separable infinite dimensional Banach space and $\mathcal{N}$ be the set of nuclear quasinilpotent operators endowed with the nuclear norm metric. Then the set of $T \in \mathcal{N}$ for which $T$ is supercyclic and $I+T$ is hypercyclic is a dense $G_{\delta}$ subset of the complete metric space $\mathcal{N}$. If additionally $X^{\prime}$ is separable, then the set of $T \in \mathcal{N}$ for which $T$ and $T^{\prime}$ are supercyclic and $I+T$ and $I+T^{\prime}$ are hypercyclic is a dense $G_{\delta}$ subset of $\mathcal{N}$.

Theorems 1.21 and 1.19 provide a large supply of dual hypercyclic operators $T$ on any infinite dimensional Banach space with separable dual.

\section{Extended Backward Shifts}

Godefroy and Shapiro [27] have introduced the notion of a generalized backward shift. Namely, a continuous linear operator $T$ on a topological vector space $X$ is called a generalized backward shift if its generalized kernel

$$
\operatorname{ker} * T=\bigcup_{n=1}^{\infty} \operatorname{ker} T^{n}
$$

is dense in $X$ and ker $T$ is one-dimensional. We introduce a more general concept. Namely, we say that $\mathrm{T}$ is an extended backward shift if

$$
\operatorname{ker}^{\dagger} T=\operatorname{span}\left(\bigcup_{n=1}^{\infty}\left(T^{n}(X) \cap \operatorname{ker} T^{n}\right)\right)
$$

is dense in $X$. From the easy dimension argument [27] it follows that if $T \in L(X)$ is a generalized backward shift, then dimker $T^{n}=n$ and $\mathrm{T}$ $\left(\operatorname{ker} T^{n+1}\right)=\operatorname{ker} T^{n}$ for each $n \in \mathbb{N}$. Hence ker $T^{n}=T^{n}\left(\operatorname{ker} T^{2 n}\right)$ and therefore $\operatorname{ker} T^{n}=T^{n}(X) \cap \operatorname{ker} T^{n}$ for any $n \in \mathbb{N}$. It follows that $\operatorname{ker}^{\star} T=\operatorname{ker}^{\dagger} T$ for a generalized backward shift. That is, any generalized backward shift is an extended backward shift.

We also consider the following analog of the concept of an extended backward shift for a $k$-tuple of operators. Let $T_{1}, \ldots, T_{k}$ be continuous linear operators on a topological vector space $X$. We say that $T=\left(T_{1}, \ldots, T_{k}\right) \in L(X)^{k}$ is a $E B S_{k}$-tuple if $T_{m} T_{j}=T_{j} T_{m}$ for any $j, m \in$ $\{1, \ldots, k\}$ and

$$
(T)=\operatorname{span}\left(\bigcup_{n \in \mathbb{N}^{k}} \kappa(n, T)\right), \text { where } \kappa(n, T)=T_{1}^{n_{1}} \ldots T_{k}^{n_{k}}\left(\bigcap_{j=1}^{k} \operatorname{ker} T_{j}^{2 n_{j}}\right),
$$

is dense in $X$.

It is easy to see that in the case of one operator (that is, $k=1$ and $\left.T=T_{1} \in L(X)\right), \kappa(n, T)=T^{n}\left(\operatorname{ker} T^{2 n}\right)=\operatorname{ker} T^{n} \cap T^{n}(X)$ and therefore the last definition is a generalization of the previous one. In order to study extended backward shifts we need to establish some properties of the backward shift on the finite dimensional space $\mathbb{K}^{2 n}$.

\section{Backward shift on $\mathbb{K}^{2 n}$} [28].

The following lemma is a modification of a lemma from reference
Lemma 2.1. For each $n \in \mathbb{N}$ and $z \in \mathbb{C} \backslash\{0\}$, the matrix $A_{n, z}=\left\{\frac{z^{j+k-1}}{(j+k-1) !}\right\}_{j, k=1}^{n}$ is invertible.

Proof. For each $n, k \in \mathbb{N}$ consider the matrix $M_{n, k}=\left\{\frac{(k+n-l) !}{(k+n-l+j-1)}\right\}_{j, l=1}^{n}$. First, we demonstrate that the determinants of $M_{n, k}$ satisfy the recurrent formula

$$
\operatorname{det} M_{n, k}=\frac{(n-1) ! k !(k+1) !}{(k+n-1) !(k+n) !} \operatorname{det} M_{n-1, k+2} \text { for } \mathrm{n} \geq 2 \text {. }
$$

The equality (2.3) for $n=2$ is trivial. Suppose now that $n \geq 3$. Subtracting the previous column from each column of $M_{n, k}$ except the first one, we see that det $M_{n, k}=\operatorname{det} N_{n, k}$ where $N_{n, k}=\left\{j \frac{(k+n-l-1) !}{(k+n-l+j) !}\right\}_{j, l=1}^{n-1}$ Dividing the $j$-th row of $N_{n, k}$ by $j$ and multiplying the $j$-th column by $a_{j}=(k+n-j+1)(k+n-j)$ for $1 \leq j \leq n$, we arrive at the matrix $M_{n-1, k+2}$. Hence

$$
\operatorname{det} M_{n, k}=\operatorname{det} M_{n-1, k+2} \prod_{j=1}^{n-1} j a_{j}^{-1}=\frac{(n-1) ! k !(k+1) !}{(k+n-1) !(k+n) !} \operatorname{det} M_{n-1, k+2},
$$

which proves (2.3). Since $\operatorname{det} M_{1, k}=1$ for each $k \in \mathbb{N}$, from (2.3), it follows that $\operatorname{det} M_{n, k} \neq 0$ for any $n, k \in \mathbb{N}$. Let now $B_{n}$ be the matrix obtained from $A_{n, 1}$ by putting the columns of $A_{n, 1}$ in the reverse order. Clearly det $A_{n, 1}=(-1)^{n-1} \operatorname{det} B_{n^{n}}$. On the other hand, multiplying the $j$-th column of $B_{n}$ by $(n-j+1)$ ! for $1 \leq j \leq n$, we get the matrix $M_{n, 1}$. Hence,

$$
\operatorname{det} A_{n, 1}=(-1)^{n-1} \operatorname{det} M_{n, 1} \prod_{j=1}^{n}(j !)^{-1} \text { for each } n \in \mathbb{N}
$$

Since $\operatorname{det} M_{n, 1} \neq 0$, we see that $\operatorname{det} A_{n, 1} \neq 0$ and therefore $A_{n, 1}$ is invertible. Finally, for any $z \in \mathbb{C}$ consider the diagonal $n \times n$ matrix $D_{n, z}$ with the entries $\left(1, z, \ldots, z^{n-1}\right)$ on the main diagonal. It is straightforward to verify that

$$
A_{\mathrm{n}, \mathrm{z}}=z D_{n, z} A_{\mathrm{n}, 1} D_{n, z} \text { for any } z \in \mathbb{C} .
$$

Since $A_{\mathrm{n}, 1}$ and $D_{n, z}$ for $z \neq 0$ are invertible, we see that $A_{\mathrm{n}, \mathrm{z}}$ is invertible for each $n \in \mathbb{N}$ and $z \in \mathbb{C} \backslash\{0\}$.

Lemma 2.2. Let $n \in \mathbb{N}$ and $e_{1}, \ldots, e_{2 n}$ be the canonical basis of $\mathbb{K}^{2 n}$ and $S \in L\left(\mathbb{K}^{2 n}\right)$ be the backward shift defined by $S e_{1}=0$ and $S e_{k}=e_{k-1}$ for $2 \leq$ $k \leq 2 n$ and $P$ the linear projection on $\mathbb{K}^{2 n}$ onto the subspace E=span $\left\{e_{1}, \ldots, e_{n}\right\}$ along $F=\operatorname{span}\left\{e_{n+1}, \ldots, e_{2 n}\right\}$. Then for any $z \in \mathbb{K} \backslash\{0\}$ and $u, v \in E$, there exists a unique $x^{z}=x^{z}(u, v) \in \mathbb{K}^{2 n}$ such that

$$
P x^{z}=u \quad \text { and } P e^{z S} x^{z}=v .
$$

Moreover, for any bounded subset $B$ of $E$ and any $\varepsilon>0$, there is $c=c(\varepsilon, B)>0$ such that

$$
\begin{aligned}
& \sup _{u, v \in B}\left|\left(x^{z}(u, v)\right)_{n+j}\right| \leq c|z|^{-j} \quad \text { for } 1 \leq j \leq n \text { and }|z| \geq \epsilon \\
& \sup _{u} v \in B\left|\left(e^{z S} x^{z}(u, v)\right)_{n+j}\right| \leq c|z|^{j} \quad \text { for } 1 \leq j \leq n \text { and }|z| \geq \epsilon .
\end{aligned}
$$

In particular, $x^{z}(u, v) \rightarrow u$ and $e^{z s} x^{z}(u, v) \rightarrow v$ as $|z| \rightarrow \infty$ uniformly for $u, v$ from any bounded subset of $E$.

Proof. Let $u, v \in E$ and $z \in \mathbb{K} \backslash\{0\}$. For $y \in \mathbb{K}^{2 n}$ we denote

$\bar{y}=\left(y_{n+1}, \ldots, y_{2 n}\right) \in \mathbb{K}^{n}$

One easily sees that (2.5) is equivalent to the vector equation 


$$
A_{n, z} \bar{x}^{z}=w^{z}
$$

where $A_{n, z}$ is the matrix from Lemma 2.1 and $w^{z}=w^{z}(u, v) \in \mathbb{K}^{2 n}$ is defined as

$$
w_{j}^{z}=v_{n-j+1}-\sum_{k=n-j+1}^{n} \frac{z^{k+j-n-1} u_{k}}{(k+j-n-1) !} \quad \text { for } 1 \leq j \leq n,
$$

provided we set $x_{j}=u_{j}$ for $1 \leq j \leq n$. According to Lemma 2.1, the matrix $A_{n z}$ is invertible for any $z \in \mathbb{K} \backslash\{0\}$ and therefore (2.8) is uniquely solvable. Thus there exists a unique $x^{z}=x(z, u, v) \in \mathbb{K}^{2 n}$ satisfying (2.5). It remains to verify the estimates (2.6) and (2.7). From (2.9) it follows that for any bounded subset $B$ of $E$ and any $\varepsilon>0$, there is $a=a(\varepsilon, B)$ such that

$$
\left|\left(w^{z}(u, v)\right) j\right| \leq a|z|^{j-1} \text { if } u, v \in B,|z| \geq \varepsilon \text { and } 1 \leq j \leq n .
$$

Recall that $D_{n, z}$ is the diagonal $n \times n$ matrix with the entries $(1, z, \ldots$, $z^{n-1}$ ) on the diagonal. Equalities (2.8) and (2.4) imply

$$
\bar{x}^{z}=A_{n, z}^{-1} w^{z}=z^{-1} D_{n, z}^{-1} A_{n, 1}^{-1} D_{n, z}^{-1} w^{z},
$$

where we use invertibility of $A_{n, 1}$ provided by Lemma 2.1. According to (2.10), the set $\left\{D_{n, z}^{-1} w^{z}(u, v):|z| \geq \epsilon, u, v \in B\right\}$ is bounded in $\mathbb{K}^{n}$. Hence the set $Q=\left\{A_{n, 1}^{-1} D_{n, z}^{-1} w^{z}(u, v):|z| \geq \epsilon, u, v \in B\right\}$ is bounded in $\mathbb{K}^{n}$. From the last display we see that

$$
\left(x^{z}(u, v)\right)_{n+j}=\bar{x}_{j}^{z} \subseteq\left\{z^{-1}\left(D_{n, z}^{-1} y\right)_{j}: y \in Q\right\} \text { if }|\mathrm{z}| \geq \epsilon, \mathrm{u}, \mathrm{v} \in \mathrm{B} .
$$

Boundedness of $Q$ implies now that (2.6) is satisfied with some $c=c_{1}(\varepsilon, B)$. Finally, since

$$
\left(e^{z S} x^{z}\right)_{n+j}=\sum_{l=n+j}^{2 n} \frac{z^{l-n-j} x_{l}^{z}}{(l-n-j) !} \text { for } 1 \leq j \leq n,
$$

there exists $c=c_{2}(\varepsilon, B)$ for which (2.7) is satisfied. Hence both (2.7) and (2.6) are satisfied with

$$
c=\max \left\{c_{1}(\varepsilon, B), c_{2}(\varepsilon, B)\right\} .
$$

The next corollary follows immediately from Lemma 2.2.

Corollary 2.3. Let $n \in \mathbb{N}, E \subseteq \mathbb{K}^{2 n}$ and $S \in L\left(\mathbb{K}^{2 n}\right)$ be as in Lemma 2.2. Then for any $u, v \in E$ and any sequence $\left\{z_{j}\right\}_{j \in \mathbb{Z}_{+}}$in $\mathbb{K}$ satisfying $\left|z_{j}\right| \rightarrow$ $\infty$, there exists a sequence $\left\{x_{j}\right\}_{j \in \mathbb{Z}+}$ of elements of $\mathbb{K}^{2 n}$ such that $x_{j} \rightarrow u$ and $e^{z j s} x_{j} \rightarrow v$ as $j \rightarrow \infty$.

Lemma 2.4. Let $n \in \mathbb{N}, E \subseteq \mathbb{K}^{2 n}$. and $S \in L\left(\mathbb{K}^{2 n}\right)$ be as in Lemma 2.2. Then for any bounded sequences $\left\{u_{j}\right\}_{j \in \mathbb{Z}_{+}}$and $\left\{v_{j}\right\}_{j \in \mathbb{Z}_{+}}$of elements of $E$, there exists a sequence $\left\{x_{j}\right\}_{j \in \mathbb{Z}+}$ of elements of $\mathbb{K}^{2 n}$ such that $x_{j}-u_{j} \rightarrow 0$ and $(I+S)^{j} x_{j}-v_{j} \rightarrow 0$ as $j \rightarrow \infty$.

Proof. It is easy to see that there is $J \in L\left(\mathbb{K}^{2 n}\right)$ such that $J$ has an upper triangular matrix, $J$ is invertible and $S=J^{-1}\left(e^{S}-I\right) J$. Indeed, $S$ and $e^{S}-I$ are similar since they are nilpotent of maximal rank $2 n-1$. Moreover, since $S$ and $e^{S}-I$ are upper triangular, the similarity operator can be chosen upper triangular and therefore $J(E) \subseteq E$.

Let now $x_{j}=J^{-1} x^{j}\left(J u_{j}, J v_{j}\right)$, where $x^{z}(u, v)$ is defined in Lemma 2.2. Since the set

$$
\left\{J u_{j}: j \in \mathbb{Z}_{+}\right\} \cup\left\{J v_{j}: j \in \mathbb{Z}_{+}\right\}
$$

is bounded and is contained in $E$ because $J(E) \subseteq E$, from Lemma 2.2 it follows that $x^{j}\left(J u_{j}, J v_{j}\right)-J u_{j} \rightarrow 0$ and $e^{j s} x^{j}\left(J u_{j}, J v_{j}\right)-J v_{j} \rightarrow 0$ as $j \rightarrow \infty$. Multiplying by $J^{-1}$, we obtain $x_{j}-u_{j} \rightarrow 0$ and $J^{-1} e^{j S} J x_{j}(I+S)^{j} x_{j}-v_{j} \rightarrow 0$ as $j \rightarrow \infty$.
In order to construct multi-parameter mixing semigroups, we need the following multi-operator version of Corollary 2.3.

Lemma 2.5. Let $k \in \mathbb{N}, n_{1}, \ldots, n_{k} \in \mathbb{N}$, for each $j \in\{1, \ldots, k\}$ let $e_{1}^{j}, \ldots, e_{2 n_{j}}^{j}$ be the canonical basis in $\mathbb{K}^{2 n_{j}}, E_{j}=\operatorname{span}\left\{e_{1}^{j}, \ldots, e_{n_{j}}^{j}\right\}$ and $S_{j} \in L\left(\mathbb{K}^{2 n_{j}}\right)$ be the backward shift: $S_{j} e_{1}^{j}=0$ and $2 \leq l \leq 2 n_{j}$. Let also $X=\mathbb{K}^{2 n_{1}} \otimes \ldots \otimes \mathbb{K}^{2 n_{k}}, E=E_{1} \otimes \ldots \otimes E_{k}$ and for $1 \leq j \leq k$,

$$
T_{j} \in L(X), T_{j}=I \otimes \ldots I \otimes S_{j} \otimes I \otimes \ldots \otimes I \text {, }
$$

where $S_{j}$ sits in the $j^{\text {th }}$ place. Finally, let $\left\{z_{m}\right\}_{m \in \mathbb{Z}+}$ be a sequence of elements of $\mathbb{K}^{k}$ with $\left|z_{m}\right| \rightarrow \infty$. Then for any $u, v \in E$, there exists a sequence $\left\{x_{m}\right\}_{m \in \mathbb{Z}+}$ of elements of $X$ such that $x_{m} \rightarrow u$ and $e^{\left\langle z_{m}, T\right\rangle} x_{m} \rightarrow v$ as $m \rightarrow \infty$, where $\langle s, T\rangle=s_{1} T_{1}+\ldots+s_{k} T_{k}$.

Proof. If the statement of the lemma is false, then there are $u, v \in E$ and a subsequence $\left\{z_{m}^{\prime}\right\}_{\mathrm{m} \in \mathbb{Z}+}$ of $\left\{z_{m}\right\}$ such that $(u, v)$ does not belong to the closure of the set $\left\{\left(x, e^{\left\langle z_{l}^{\prime}, T\right\rangle} x\right): x \in X, m \in \mathbb{Z}_{+}\right\}$.

Let $\overline{\mathbb{K}}=\mathbb{K} \cup\{\infty\}$ be the one-point compactification of $\mathbb{K}$. Since $\overline{\mathbb{K}}^{k}$ is compact and metrizable, we can pick a convergent in $\overline{\mathbb{K}}^{k}$ subsequence $\left\{z_{m}{ }^{\prime \prime}\right\}$ of $\left\{z_{m}{ }^{\prime}\right\}$. Clearly the statement of the lemma remains false with $\left\{z_{m}\right\}$ replaced by $\left\{z_{m}{ }^{\prime \prime}\right\}$. That is, it suffices to consider the case when $\left\{z_{m}\right\}$ converges in $\overline{\mathbb{K}}^{k}$.

Thus without loss of generality, we can assume that $\left\{z_{m}\right\}$ converges to $w \in \overline{\mathbb{K}}^{k}$. Let $C=\left\{j: w_{j}=\infty\right\}$. Since $\left|z_{m}\right| \rightarrow \infty$, the set $C$ is non-empty. Without loss of generality, we may also assume that $C=\{1, \ldots, r\}$ with 1 $\leq r \leq k$.

Denote by $\Sigma$ the set of $(u, v) \in X \times X$ such that there exists a sequence $\left\{x_{m}\right\}_{m \in \mathbb{Z}_{+}}$of elements of $X$ for which $x_{\mathrm{m}} \rightarrow u$ and $e^{\left\langle z_{m}, T\right\rangle_{m}} x_{m} \rightarrow v$ as $m \rightarrow \infty$. We have to demonstrate that $E \times E \subseteq \Sigma$. Let $u_{j} \in E_{j}$ for $1 \leq j \leq \mathrm{k}$ and $\mathrm{u}=\mathrm{u}_{1} \otimes \ldots \otimes u_{k}$. By Corollary 2.3, for $1 j \leq r$, there exist sequences $\left\{x_{j, m}\right\}_{m \in \mathbb{Z}+}$ and $\left\{y_{j, m}\right\}_{m \in \mathbb{Z}_{+}}$of elements of $\mathbb{K}^{2 n_{j}}$ such that

$$
\left.\left.x_{j, m} \rightarrow 0, e^{\left(z_{m}\right)}\right)_{j} x_{j, m} \rightarrow u_{j}, y_{j, m} \rightarrow u_{j} \text { and } e^{\left(z_{m}\right)}\right)_{j} S_{j} y_{j, m} \rightarrow 0 \text { as } m \rightarrow \infty \text { for } 1 \leq j \leq r .
$$

Now we put $x_{j, m}=e^{-w_{j} S_{j}} u_{j}$ and $y_{j, m}=u_{j}$ for $r<j \leq k$ and $m \in \mathbb{Z}_{+}$. Consider the sequences $\left\{x_{m}\right\}_{m \in \mathbb{Z}_{+}}$and $\left\{y_{m}\right\}_{m \in \mathbb{Z}_{+}}$of elements of $X$ defined by the formula $x_{m}=x_{1, m} \otimes \ldots \otimes x_{k, m}$ and $y_{m}=y_{1, m} \otimes \ldots \otimes y_{k, m}$. According to the definition of $x_{m}$ and $y_{m}$ and the above display, $x_{m} \rightarrow 0$ and $y_{m} \rightarrow u$. Indeed, $x_{m} \rightarrow 0$ because for any $j$, the sequence $x_{j, m}$ is bounded and $x_{1, m}$ $\rightarrow 0$. Similarly, taking into account that $\left(z_{m}\right)_{j} \rightarrow w_{j}$ for $j>r$, we see that $e^{\left\langle z_{m}, T\right\rangle} x_{m} \rightarrow$ uand $e^{\left\langle z_{m}, T\right\rangle} y_{m} \rightarrow 0$. Hence $(u, 0) \in \Sigma$ and $(0, u) \in \Sigma$. Thus

$\left(\{0\} \times E_{0}\right) \cup\left(E_{0} \times\{0\}\right) \subseteq \Sigma$,

where $E_{0}=\left\{u_{1} \otimes \ldots \otimes u_{k}: u_{j} \in E j, 1 \leq j \leq k\right\}$. On the other hand, it is easy to see that the linear span of the set $\left(\{0\} \times E_{0}\right) \cup\left(E_{0} \times\{0\}\right)$ is exactly $E \times E$. Since $\Sigma$ is a linear space, the above display implies that $E \times E \subseteq \Sigma$.

For applications it is more convenient to reformulate the above lemma in the coordinate form.

Corollary 2.6. Let $k \in \mathbb{N}, n_{1}, \ldots, n_{k} \in \mathbb{N}$, for each $j \in\{1, \ldots, k\}$ let $N_{j}=\left\{1, \ldots, 2 n_{j}\right\}, Q_{j}=\left\{1, \ldots, n_{j}\right\}$. Consider the sets $M=N_{1} \times \ldots \times N_{k}, M_{0}=Q_{1} \times \ldots$ $\times Q_{k}$ and let $\left\{e_{m}: m \in M\right\}$ be the canonical basis of the finite dimensional vector space $X=\mathbb{K}^{M}$. Let $E=\operatorname{span}\left\{e_{m}: m \in M_{0}\right\}$ and for $1 \leq j \leq k, T_{j} \in$ $L(X)$ be the operator acting on the canonical basis in the following way: $T_{j} e_{m}=0$ if $m_{j}=1, T_{j} e_{m}=e_{m}{ }^{\prime}$ if $m_{j}>1$, where $m_{l}^{\prime}=m^{\prime}$ if $l \neq j, m_{j}^{\prime}=m_{j}-1$. Then for any sequence $\left\{z_{m}^{m}\right\}_{m \in \mathbb{Z}+}$ of elements of $\mathbb{K}^{k}$ with $\left|z_{m}\right| \rightarrow \infty$ and any $u, v \in$ $E$, there exists a sequence $\left\{x_{m}\right\}_{m \in \mathbb{Z}_{+}}$of elements of $X$ such that $x_{m} \rightarrow u$ and $e^{\left\langle z_{m}, T\right\rangle} x_{m} \rightarrow v$ as $m \rightarrow \infty$. 


\section{The key lemmas}

Lemma 2.7. Let $X$ be a Hausdorff topological vector space, $k \in \mathbb{N}$, $n=\left(n_{1}, \ldots, n_{k}\right) \in \mathbb{N}^{k}$ and $A=\left(A_{1}, \ldots, A_{k}\right) \in L(X)^{k}$ be such that $A_{j} A_{l}=A_{l} A_{i}$ for any $l, j \in\{1, \ldots, k\}$. Then for each $x$ from the space $\kappa(n, A)$ defined by $(2.2)$, there exists a common finite dimensional invariant subspace for $A_{1}, \ldots, A_{k}$ such that for any sequence $\left\{z_{m}\right\}_{m \in \mathbb{Z}+}$ of elements of $\mathbb{K}^{k}$ with $\left|z_{m}\right| \rightarrow \infty$ there exist sequences $\left\{x_{m}\right\}_{m \in \mathbb{Z}+},\left\{y_{m}\right\}_{m \in \mathbb{Z}+}$ of elements of $Y$ for which

$$
\begin{aligned}
& x_{m} \rightarrow 0, e^{\left\langle z_{m}, A\right\rangle} x_{m} \rightarrow x, y_{m} \rightarrow x, e^{\left\langle z_{m}, A\right\rangle} y_{m} \rightarrow 0 \text { as } m \rightarrow \infty, \\
& \text { where }\langle s, A\rangle=\left.\left(s_{1} A_{1}+\ldots+s_{k} A_{k}\right)\right|_{Y} .
\end{aligned}
$$

Proof. Since $x \in \kappa(n, T)$, there exists $\mathrm{y} \in \mathrm{X}$ such that $x=A^{n_{1}} \ldots A^{n_{k}} y$ and $A^{2 n_{j}} y=0$ for $1 \leq j \leq k$. For each $j \in\{1, \ldots, k\}$ let $\mathrm{N}_{j}=\left\{1, \ldots, 2 n_{j}\right\}$ and $Q_{j}=\left\{1, \ldots, n_{j}\right\}$. Denote $M=N_{1} \times \ldots \times N_{k}, M_{0}=Q_{1} \times \ldots \times Q_{k}$. For any $l \in M$, let $h_{l}=A^{2 n_{1}-l_{1}} \ldots A^{2 n_{k}-l_{k}} y$ and let $Y=\operatorname{span}\left\{h_{l}: l \in M\right\}$. Clearly $Y$ is finite dimensional. It is also straightforward to verify that $A h_{l}=0$ if $l_{j}=1$ and $A$ $h_{l}=h_{l^{\prime}}$ if $l_{j}>1$, where $l_{r}^{\prime}=l_{r}$ for $r \neq j, l_{j}^{\prime}=l_{i}-1,1 \leq j \leq k_{0}$. It follows that $Y$ is invariant for $A_{j}$ for $1 \leq j \leq k$. Consider the linear operator $J: \mathbb{K}^{M} \rightarrow Y$ defined on the canonical basis by the formulas $J e_{l}=h_{l}$ for $l \in \mathrm{M}$. Let also $E=\operatorname{span}\left\{e_{l}: l \in M_{0}\right\}$ and $T_{j} \in L\left(\mathbb{K}^{\mathrm{M}}\right)$ be the operators from Corollary 2.6. Taking into account the definition of $T_{j}$ and the action of $A_{j}$ on $h_{p}$, we see that $A_{j} J=J T_{j}$ for $1 \leq j \leq k$. Clearly $n=\left(n_{1}, \ldots, n_{k}\right) \in M_{0}$ and therefore $e_{n}$ $\in E$. Since $x=A^{n_{1}} \ldots A^{n_{k}} y$, we also see that $x=h_{n}$. According to Corollary 2.6, there exist sequences $\left\{u_{m}\right\}_{m \in \mathbb{Z}+}$ and $\left\{v_{m}\right\}_{m \in \mathbb{Z}_{+}}$of elements of $\mathbb{K}^{\mathrm{M}}$ such that $u_{m} \rightarrow e_{n}, e^{\left\langle z_{m}, T\right\rangle} u_{m} \rightarrow 0, v_{m} \rightarrow 0$ and $e^{\left\langle z_{m}, T\right\rangle} u_{m} \rightarrow e_{n}$ asm $\rightarrow \infty$. Now let $y_{m}=J u_{m}$ and $x_{m}=J v_{m}$ for $\mathrm{m} \in \mathbb{Z}_{+}$. Then $\left\{x_{m}\right\}$ and $\left\{y_{m}\right\}$ are sequences of elements of $Y$. From the intertwining relations $A J=J T_{j}$ and the fact that $\mathbb{K}^{M}$ and $Y$ are finite dimensional, it follows that $x_{m} \rightarrow J 0=0, y_{m} \rightarrow J e_{n}=x$, $e^{\left\langle z_{m}, A\right\rangle} x_{m} \rightarrow J e_{n}=x, e^{\left\langle z_{m}, T\right\rangle} y_{m} \rightarrow J 0=0$. Thus (2.11) is satisfied.

Let $X$ be a topological vector space and $T=\left(T_{1}, \ldots, T_{k}\right) \in L(X)^{k}$. We write $T \in \varepsilon(k, X)$ if for $\infty$ any $z \in \mathbb{K}^{k}$, the series $\sum_{n=0}^{\infty} \frac{1}{n !}\langle z, T\rangle^{n} x$ converges in $X$ and the map $(z, x) \rightarrow e^{\langle z, T\rangle} x$ from $\mathbb{K}^{k} \times X$ to $X$ is separately continuous, where $\langle z, T\rangle=z_{1} T_{1}+\ldots+z_{k} T_{k}$ and $e^{S} x=\sum_{n=0}^{\infty} \frac{1}{n !} S^{n} x$. The following proposition is an elementary exercise. We leave its proof for the reader.

Proposition 2.8. Let $k \in \mathbb{N}, X$ be a locally convex space and $T \in$ $\varepsilon(k, X)$. Assume also that $T_{j} T_{m}=T_{m} T_{j}$ for any $m, j \in\{1, \ldots, k\}$. Then $\left\{e^{\langle z, T\rangle}\right\}_{z \in \mathbb{K}^{k}}$ is a strongly continuous operator group. Moreover, if $\mathbb{K}=\mathbb{C}$, then the map $z \mapsto e^{\langle z, T\rangle} x$ from $\mathbb{C}^{k}$ to $X$ is holomorphic for each $x \in X$.

Remark 2.9. It is worth noting that the semigroup property $e^{\langle z+w, T\rangle}=e^{\langle z, T\rangle} e^{\langle w, T\rangle}$ fails if the operators $T_{j}$ are not pairwise commuting.

Corollary 2.10. Let $X$ be a locally convex space, $k \in \mathbb{N}, n_{1}, \ldots, n_{k} \in \mathbb{N}$ and $A=\left(A_{1}, \ldots, A_{k}\right) \in \varepsilon(k, X)$ be such that $A_{j} A_{l}=A_{l} A_{j}$ for any $l, j \in\{1, \ldots$, $k\}$. Then for each $x, y$ from the space $k^{\dagger} r^{\dagger}(A)$ defined by (2.2) and any sequence $\left\{Z_{m}\right\}_{m \in \mathbb{Z}+}$ of elements of $\mathbb{K}^{k}$ with $\left|z_{m}\right| \rightarrow \infty$, there exist a sequence $\left\{u_{m}\right\}_{m \in \mathbb{Z}_{+}}$in $X$ such that $u_{m} \rightarrow x$ and $e^{\left\langle z_{m}, A\right\rangle} u_{m} \rightarrow y$ as $m \rightarrow \infty$.

Proof. Fix a sequence $\left\{z_{m}\right\}_{m \in \mathbb{Z}_{+}}$of elements of $\mathbb{K}^{k}$ with $\left|\mathrm{z}_{\mathrm{m}}\right| \rightarrow \infty$. Let $\Sigma$ be the set of $(x, y) \in X \times X$ for which there exists a sequence $\left\{u_{m}\right\}_{n \in \mathbb{Z}}$ in $X$ such that $u_{m} \rightarrow x$ and $e^{\left\langle z_{m}, A\right\rangle} u_{m} \rightarrow y$ as $m \rightarrow \infty$. According to Lemma
2.7, $\kappa(\mathrm{n}, \mathrm{A}) \times\{0\} \subseteq \Sigma$ and $\{0\} \times \kappa(\mathrm{n}, \mathrm{A}) \subseteq \Sigma$ for any $n=\left(n_{1}, \ldots, n_{k}\right) \in \mathbb{N}^{\mathrm{k}}$, where the space $\kappa(n, A)$ is defined by (2.2). On the other hand, from the definition of $\Sigma$ it is clear that $\Sigma$ is a linear subspace of $X \times X$. Thus

$$
\left.\operatorname{ker}^{\dagger}(A) \times \operatorname{ker}^{\dagger}(A)=\operatorname{span}\left(\bigcup_{n \in \mathbb{N}^{k}}(\kappa(n, A) \times\{0\}) \cup(\{0\} \times \kappa(n, A))\right)\right) \subseteq \Sigma .
$$

Hence $(x, y) \in \Sigma$ for any $x, y \in \operatorname{ker} \dagger(A)$.

Lemma 2.11. Let $X$ be a topological vector space, $z \in \mathbb{K},|z|=1, A \in$ $L(X), m \in \mathbb{N}$ and $x \in A^{m}(X) \cap \operatorname{ker} A^{m}$. There exist sequences $\left\{u_{k}\right\}_{k \in \mathbb{Z}+}$ and $\left\{v_{k}\right\}_{k \in \mathbb{Z}_{+}}$of elements of $X$ such that

$$
u_{k} \rightarrow 0, z^{k}(I+A)^{k} u_{k} \rightarrow x, v_{k} \rightarrow x, z^{k}(I+A)^{k} v_{k} \rightarrow 0 \text { as } k \rightarrow \infty .
$$

Proof. If $x=0$, we can take $u_{k}=v_{k}=0$, so we may assume that $x \neq 0$. Let $n$ be the smallest positive integer for which $A^{n} x=0$. Since $A^{m} x=0$, we have $n \leq m$. Hence $x \in A^{m}(X) \subseteq A^{n}(X)$. Thus we can pick $w \in X$ such that $A^{n} w=x$. Denote

$$
h j=A^{2 n-j} w \text { for } 1 \leq j \leq 2 n \text { and } Y=\operatorname{span}\left\{h_{1}, \ldots, h_{2 n}\right\} \text {. }
$$

Clearly $A h_{j}=h_{j-1}$ for $2 \leq j \leq 2 n, h_{n}=A^{n} h_{2 n}=A^{n} w=x$ and $A h_{1}=A^{2 n} h_{2 n}=A^{n} x=0$. By definition of $\mathrm{n}$ we have $h_{1}=A^{2 n-1} h_{2 n}=A^{n-1} x \neq 0$. In particular $A(Y) \subseteq Y,\left(\left.A\right|_{\mathrm{y}}\right)^{2 \mathrm{n}}=0$ and $\left(\left.A\right|_{\mathrm{y}}\right)^{2 \mathrm{n}-1} \neq 0$. Since the order of nilpotency of a nilpotent operator on $Y$ cannot exceed the dimension of $Y$, we have $\operatorname{dim} Y \geq 2 n$. On the other hand $Y$ is the span of the $2 n$-elements set $\left\{h_{1}, \ldots, h_{2 n}\right\}$. Hence $\left\{h_{1}, \ldots, h_{2 n}\right\}$ is a linear basis of $Y$. Thus there exists a unique linear isomorphism $J: \mathbb{K}^{2 \mathrm{n}} \rightarrow Y$ such that $J e_{k}=h_{k}$ for $1 \leq j \leq 2 n$. Since $A h_{j}=h_{j-1}$ for $2 \leq j \leq 2 n$ and $A h_{1}=0$, we have $\left.A\right|_{y}=J S J^{-1}$, where $S \in L\left(\mathbb{K}^{2 n}\right)$ is the backward shift operator from Lemma 2.2. Applying Lemma 2.4 with $u^{m}=(0, \ldots, 0,1)$ and $v^{m}=(0, \ldots, 0,0)$, we find that there exists a sequence $\left\{g_{k}\right\}_{\mathrm{k} \in \mathbb{Z}+}$ of vectors in $\mathbb{K}^{2 n}$ such that $\mathrm{g}_{k}$ $\rightarrow \mathrm{e}_{\mathrm{n}}$ and $(I+S)^{\mathrm{k}} g_{k} \rightarrow 0$ as $k \rightarrow \infty$. Applying Lemma 2.4 with $u^{m}=(0, \ldots, 0$, $0)$ and $v^{m}=\left(0, \ldots, 0, z^{-m}\right)$, there exists also a sequence $\left\{f_{\mathrm{k}}\right\}_{\mathrm{k} \in \mathbb{Z}+}$ of vectors in $\mathbb{K}^{2 \mathrm{n}}$ such that $f_{k} \rightarrow 0$ and $z^{k}(\mathrm{I}+\mathrm{S})^{\mathrm{k}} f_{k} \rightarrow e_{n}$ as $k \rightarrow \infty$. Define now $u_{k}=J f_{k}$ and $v_{k}=J g_{k}$ for $\mathrm{k} \in \mathbb{Z}_{+}$. Since $Y$ and $\mathbb{K}^{2 \mathrm{n}}$ are finite dimensional, we see that

$$
\begin{gathered}
v_{k} \rightarrow J e_{n}=x, u_{k} \rightarrow 0,(I+A)^{k} v_{k}=J(I+S)^{k} J^{-1} J g_{k}=J(I+S)^{k} g_{k} \rightarrow 0, \\
z^{k}(I+A)^{k} u_{k}=z^{k} J(I+S)^{k} J^{-1} J f_{k}=z^{k} J(I+S)^{k} f_{k} \rightarrow J e_{n}=x \text { as } k \rightarrow \infty .
\end{gathered}
$$

Thus the sequences $\left\{u_{k}\right\}$ and $\left\{v_{k}\right\}$ satisfy the desired conditions.

The following corollary of Lemma 2.11 seems to be of independent interest.

Theorem 2.12. Let $X$ be a topological vector space, $T \in L(X)$ and $\Lambda(T)$ be the set defined in (1.1). Then for any $u, v \in \Lambda(T)$, there exists a sequence $\left\{X_{k}\right\}_{k \in \mathbb{Z}_{+}}$in $X$ such that $x_{k} \rightarrow u$ and $T^{k} x_{k} \rightarrow v$ as $k \rightarrow \infty$.

Proof. Let $\Sigma$ be the set of pairs $(x, y) \in X \times X$ for which there exists a sequence $\left\{X_{k}\right\}_{k \in \mathbb{Z}_{+}}$of elements of $X$ such that $x_{k} \rightarrow x$ and $T^{k} x_{k} \rightarrow y$. Pick $z \in \mathbb{K}$ with $|z|=1$ and $n \in \mathbb{N}$ and let

$x \in \operatorname{ker}(T-z I)^{n} \cap(T-z I)^{n}(X)=\operatorname{ker} A^{n} \cap A^{n}(X)$, where $A=z^{-1} T-I$.

By Lemma 2.11, there exist sequences $\left\{u_{k}\right\}_{k \in \mathbb{Z}_{+}}$and $\left\{v_{k}\right\}_{k \in \mathbb{Z}_{+}}$in $X$ satisfying (2.12). Since $I+A=\mathrm{z}^{-1} \mathrm{~T}$, (2.12) can be rewritten in the following way:

$$
v_{k} \rightarrow x, T^{k} v_{k} \rightarrow 0, u_{k} \rightarrow 0, T^{k} u_{k} \rightarrow x \text { as } k \rightarrow \infty .
$$

This shows that for any $n \in \mathbb{N}$ and any $\mathrm{z} \in \mathbb{K}$ with $|z|=1$,

$$
\left(\operatorname{ker}(T-z I)^{n} \cap(T-z I)^{n}(X)\right)_{\times\{0\} \subseteq \Sigma} \text { and }\{0\} \times\left(\operatorname{ker}(T-z I)^{n} \cap(T-z I)^{n}(X)\right)_{\subseteq \Sigma} .
$$

On the other hand, the fact that $\Sigma$ is a linear subspace of $X \times X$, the definition of $\Lambda(T)$ and the above display imply that $\Lambda(T) \times \Lambda(T) \subseteq \Sigma$. 


\section{Mixing semigroups and extended backward shifts}

We start by proving Proposition 1.1. The next observation is Proposition 1 in reference [28].

Proposition G. Let $X$ be a topological space and $\mathcal{F}=\left\{T_{\alpha}: \alpha \in A\right\}$ be a family of continuous maps from $X$ to $X$ such that $T_{\alpha} T_{\beta}=T_{\beta} T_{\alpha}$ for any $\alpha, \beta \in A$ and $T_{\alpha}(X)$ is dense in $X$ for any $\alpha \in A$. Then the set of universal elements for $\mathcal{F}$ is either empty or dense in $X$.

The following general theorem can be found in reference [28].

Theorem U. Let X be a Baire topological space, Y a second countable topological space and $\left\{T_{a}: a \in A\right\}$ a family of continuous maps from $X$ into $Y$. Then the following assertions are equivalent:

$\left(\mathrm{U}_{1}\right)$ The set of universal elements for $\left\{T_{a}: a \in A\right\}$ is dense in $X$; of $X$;

$\left(\mathrm{U}_{2}\right)$ The set of universal elements for $\left\{T_{a}: a \in A\right\}$ is a dense $G_{\delta}$-subset

\section{$\left(\mathrm{U}_{3}\right)$ The set $\left\{\left(x, T_{a} x\right): x \in X, a \in A\right\}$ is dense in $X \times Y$.}

Proof of Proposition 1.1. Assume that $\left\{T_{t}\right\}_{t \in A}$ is hereditarily hypercyclic. That is, $\left\{T_{t_{n}}: n \in \mathbb{Z}_{+}\right\}$is universal for any sequence $\left\{t_{n}\right\}_{n \in \mathbb{Z}_{+}}$of elements of $A$ such that $\left|t_{n}\right| \rightarrow \infty$. Applying this property to the sequence $t_{n}=n t$ with $t \in A,|t|>0$, we see that $T_{t}$ is hypercyclic. Since any hypercyclic operator has dense range [28], we get that $T_{t}(X)$ is dense in $X$ for any $t \in A$ with $|t|>0$. We proceed by reasoning ad absurdum. Assume that $\left\{T_{t}\right\}_{t} \in$ is non-mixing. Then there exist nonempty open subsets $U$ and $V$ of $X$ and a sequence $\left\{t_{n}\right\}_{n \in \mathbb{Z}_{+}}$of elements of $A$ such that $\left|t_{n}\right| \rightarrow \infty$ and $\left|t_{n}\right|>0, T_{t_{n}}(U) \cap V=\varnothing$ for each $n \in \mathbb{Z}+$. Since each $T_{t_{n}}$ has dense range and $T_{t_{n}}$ commute with each other, Proposition $G$ implies that the set $W$ of universal elements of $\left\{T_{t_{n}}: n \in \mathbb{Z}_{+}\right\}$is either empty or dense in $X$. Since $\left\{T_{t_{n}}: n \in \mathbb{Z}_{+}\right\}$is universal, $W$ is non-empty and therefore dense in $X$. Hence we can pick $x \in W \cap U$. Since $x$ is universal for $\left\{T_{t_{n}}: n \in \mathbb{Z}_{+}\right\}$, there is $n \in \mathbb{Z}_{+}$for which $T_{t_{n}} x \in V$. Hence $T_{t_{n}} x \in T_{t_{n}}(U) \cap V=\varnothing$. This contradiction completes the proof of (1.1.1).

Next, assume that $X$ is Baire separable and metrizable, $\left\{T_{t}\right\}_{t} \in_{A}$ is mixing and $\left\{t_{n}\right\}_{n \in \mathbb{Z}_{+}}$is a sequence of elements of $A$ such that $\left|t_{n}\right| \rightarrow \infty$. From the definition of mixing it follows that for any non-empty open subsets $U$ and $V$ of $X, T_{t_{n}}(U) \cap V \neq \varnothing$ for all sufficiently large $n \in \mathbb{Z}_{+}$ Hence $\left\{\left(x, T_{t_{n}} x\right): x \in X, n \in \mathbb{Z}_{+}\right\}$is dense in $X \times X$. By Theorem $\mathrm{U}$, $\left\{T_{t_{n}}: n \in \mathbb{Z}_{+}\right\}$is universal.

Theorem 2.13. Let $X$ be a topological vector space, $k \in \mathbb{N}$ and $A=\left(A_{1}, \ldots, A_{k}\right) \in \varepsilon(k, X)$ be a $E B S_{k}$-tuple. Then the strongly continuous group $\left\{e^{\langle z, A\rangle}\right\}_{z \in \mathbb{K}^{k}}$ is mixing. If additionally $X$ is Baire separable and metrizable, $\left\{e^{\langle z, A\rangle}\right\}_{z \in \mathbb{K}^{k}}$ is hereditarily hypercyclic.

Proof. Assume that $\left\{e^{\langle z, A\rangle}\right\}_{z \in \mathbb{K}^{k}}$ is non-mixing. Then we can find nonempty open subsets $U$ and $V$ of $X$ and a sequence $\left\{z_{m}\right\}_{m \in \mathbb{Z}_{+}}$in $\mathbb{K}^{k}$ such that $\left|z_{m}\right| \rightarrow \infty$ as $m \rightarrow \infty$ and $e^{\left\langle z_{m}, A\right\rangle}(U) \cap V=\varnothing$ for each $m \in \mathbb{Z}_{+}$. Let $\Sigma$ be the set of pairs $(x, y) \in X \times X$ for which there exists a sequence $\left\{x_{m}^{+}\right\}_{m \in \mathbb{Z}_{+}}$of elements of $X$ such that $\mathrm{x}_{\mathrm{m}} \rightarrow \mathrm{x}$ and $e^{\left\langle z_{m}, A\right\rangle} x_{m} \rightarrow y$. According to Corollary $2.10, \operatorname{ker}^{\dagger}(A) \times \operatorname{ker}^{\dagger}(A) \subseteq \Sigma$. Since $A=\left(A_{1}, \ldots, A_{\mathrm{k}}\right)$ is a $\mathrm{EBS}_{k}$-tuple, $\operatorname{ker}^{\dagger}(A)$ is dense in $X$ and therefore $\Sigma$ is dense in $X \times X$. In particular, $\Sigma$ meets $U$ $\times V$, which is not possible since $e^{\left\langle z_{m}, A\right\rangle}(U) \cap V=\varnothing$ for any $m \in \mathbb{Z}_{+}$. This contradiction shows that $\left\{e^{\langle z, A\rangle}\right\}_{z \in \mathbb{K}^{k}}$ is mixing. If $X$ is Baire separable and metrizable, Proposition 1.1 implies that $\left\{e^{\langle z, A\rangle}\right\}_{z \in \mathbb{K}^{k}}$ is hereditarily hypercyclic.

It is easy to see that if $X$ is a Banach space and $k \in \mathbb{N}$, then $L(X)^{k}=\varepsilon$ $(k, X)$. Moreover, each operator group of the shape $\left\{e^{\langle z, A\rangle}\right\}_{z \in \mathbb{K}^{k}}$ is uniformly continuous. Hence, we get the following corollary of Theorem 2.13.

Corollary 2.14. Let $X$ be a separable Banach space and $\left(A_{1}, \ldots, A_{k}\right)$ $\in L(X)^{k}$ be a $E B S_{k}$-tuple. Then $\left\{e^{\langle z, A\rangle}\right\}_{z \in \mathbb{K}^{k}}$ is a hereditarily hypercyclic uniformly continuous group.

\section{$\ell_{1}$-sequences, equicontinuous sets and the class $\mathfrak{M}$}

Lemma 3.1. Let $Y_{0}$ and $Y_{1}$ be closed linear subspaces of a locally convex space $Y$ such that $Y_{0} \subset Y_{1}$ and the topology of $Y_{1} / Y_{0}$ is not weak. Then there is a sequence $\left\{f_{n}: n \in \mathbb{Z}_{+}\right\}$in $Y^{\prime}$ such that

(3.1.1) $\phi \subseteq\left\{\left\{f_{n}(y)\right\}_{n \in \mathbb{Z}_{+}}: y \in Y_{1}\right\}$;

(3.1.2) $\left.f_{n}\right|_{Y_{0}}=0$ for each $n \in \mathbb{Z}_{+}$;

(3.1.3) $\left\{f_{n}: n \in \mathbb{Z}_{+}\right\}$is uniformly equicontinuous.

Proof. Since the topology of $Y_{1} / Y_{0}$ is not weak, there exists a continuous seminorm $\tilde{p}$ on $Y_{1} / Y_{0}$ such that the closed linear space ker $\tilde{p}=\tilde{p}^{-1}(0)$ has infinite codimension in $Y_{1} / Y_{0}$. Clearly the seminorm $p$ on $Y_{1}$ defined by the formula $p(\mathrm{y})=\tilde{p}\left(y+Y_{0}\right)$ is also continuous and ker $p$ has infinite codimension and contains $Y_{0}$. In particular the space $Y_{p}=Y_{1} / \operatorname{ker} p$ endowed with the norm $\|x+\operatorname{ker} p\|=p(x)$ is an infinite dimensional normed space. Hence we can choose sequences $\left\{y_{n}\right\}_{n \in \mathbb{Z}}$ in $Y_{1}$ and $\left\{\mathrm{g}_{n}\right\}_{n \in \mathbb{Z}_{+}}$in $Y_{p}^{\prime}$ such that $\left\|g_{n}\right\| \leq 1$ for each $n \in \mathbb{Z}_{+}$and $g_{n}\left(y_{k}\right.$ $+\operatorname{ker} p)=\delta_{n k}$ for $n, k \in \mathbb{Z}^{p}$, where $\delta_{n, k}$ is the Kronecker $\delta$ (every infinite dimensional normed space admits a biorthogonal sequence). Now let the functionals $h_{n}: Y_{1} \rightarrow \mathbb{K}$ be defined by the formula $h_{n}(y)=\mathrm{g}_{\mathrm{n}}\left(\mathrm{y}_{k}+\right.$ ker $p$ ). The above properties of the functionals $g_{n}$ can be rewritten in terms of $h_{n}$ in the following way

$$
\left|h_{n}(y)\right| \leq p(y) \text { and } h_{n}\left(y_{k}\right)=\delta_{n, k} \text { for any } n, k \in \mathbb{Z}+\text { and } y \in Y_{1} .
$$

Since any continuous seminorm on a subspace of a locally convex space extends to a continuous seminorm on the entire space $[17,29]$, we can find a continuous seminorm $q$ on $Y$ such that $\left.q\right|_{Y_{1}}=p$.

Applying the Hahn-Banach theorem, we can find $f_{\mathrm{n}} \in Y^{\prime}$ for $n \in$ $\mathbb{Z}_{+}$such that

$$
\left.f_{n}\right|_{Y_{1}}=h_{n} \text { and }\left|f_{n}(y)\right| \leq q(y) \text { for any } \mathrm{n} \in \mathbb{Z}_{+} \text {and } \mathrm{y} \in \mathrm{Y} .
$$

From the last two displays we have $f_{\mathrm{n}}\left(y_{k}\right)=\delta_{\mathrm{n}, \mathrm{k}}$, which implies (3.1.1) since $y_{k} \in Y_{1}$. From the inequality in the above display it follows that each $\left|f_{n}\right|$ is bounded by 1 on the unit ball $W$ of the seminorm $q$. Since $W$ is a neighborhood of zero in $Y$, condition (3.1.3) is satisfied. Since $Y_{0}$ $\subseteq \operatorname{ker} p \subseteq \operatorname{ker} q$, from the inequality $\left|f_{n}(y)\right| \leq q(y)$ it follows that each $f_{n}$ vanishes on $Y_{0}$. That is, (3.1.2) is satisfied.

Applying Lemma 3.1 with $Y_{0}=0$ and $Y_{1}=Y$, we obtain the following corollary.

Corollary 3.2. Let $Y$ be a locally convex space, whose topology is not weak. Then there exists a linearly independent sequence $\left\{f_{n}: n\right.$ $\left.\in \mathbb{Z}_{+}\right\}$in $Y^{\prime}$ such that $\left\{f_{n}: n \in \mathbb{Z}_{+}\right\}$is uniformly equicontinuous and $\varphi \subseteq\left\{\left\{f_{n}(y)\right\}_{n \in \mathbb{Z}_{+}}: y \in Y\right\}$.

Recall that a subset $D$ of a locally convex space $X$ is called a disk if $D$ is bounded, convex and balanced (=is stable under multiplication by any $\lambda \in \mathbb{K}$ with $|\lambda| \leq 1)$. The symbol $X_{D}$ stands for the space span $(D)$ endowed with the norm being the Minkowskii functional of the set $D$. Boundedness of $D$ implies that the topology of $X_{D}$ is stronger than the one inherited from $X$. A disk $D$ in $X$ is called a Banach disk if the normed space $X_{D}$ is complete. It is well-known that a sequentially complete disk is a Banach disk, see, for instance, [29]. In particular, a 
compact or a sequentially compact disk is a Banach disk. We say that $D$ is a Banach s-disk in $X$ if $D$ is a Banach disk and the Banach space $X_{D}$ is separable.

Lemma 3.3. Let $\left\{x_{n}\right\}_{n \in \mathbb{Z}_{+}}$be an $\ell_{1}$-sequence in a locally convex space $X$. Then the set

$$
K=\left\{\sum_{n=0}^{\infty} a_{n} x_{n}: a \in \ell_{1},\|a\|_{1} \leq 1\right\}
$$

is a compact and metrizable disk. Moreover, $K$ is a Banach s-disk and $E=\operatorname{span}\left\{x_{n}: n \in \mathbb{Z}_{+}\right\}$is dense in the Banach space $X_{K}$

Proof. Let $Q=\left\{a \in \ell_{1}:\|\mathrm{a}\| 1 \leq 1\right\}$ be endowed with the coordinatewise convergence topology. It is easy to see that $Q$ is a metrizable compact topological space as a closed subspace of $\mathbb{D}^{\mathbb{Z}}$, where $D=\{z \in \mathbb{K}:|z| \leq 1\}$. Obviously, the map $\varphi: Q \rightarrow K, \Phi(a)=\sum_{n=0}^{\infty} a_{n} x_{n}$ is onto. It is also easy to see that $\varphi$ is continuous. Indeed, let $p$ be a continuous seminorm on $X$, $a \in Q$ and $\varepsilon>0$. Since $x_{n} \rightarrow 0$, there is $m \in \mathbb{Z}_{+}$such that $p\left(x_{n}\right) \leq \varepsilon$ for $n>$ $m$. Let $\delta=\varepsilon\left(1+p\left(x_{0}\right)+\ldots+p\left(x_{m}\right)\right)^{-1}$ and $W=\left\{b \in Q:\left|a_{j}-b_{j}\right|<\delta\right.$ for $0 \leq j$ $\leq m\}$. Then $W$ is a neighborhood of a in $Q$ and for each $b \in W$ we have

$$
p(\Phi(b)-\Phi(a))=p\left(\sum_{n=0}^{\infty}\left(b_{n}-a_{n}\right) x_{n}\right) \leq \sum_{n=0}^{\infty} b_{n}-a_{n} \mid p\left(x_{n}\right) .
$$

Taking into account that $p\left(x_{n}\right)<\varepsilon$ for $n>m$ and $\left|\mathrm{a}_{\mathrm{n}}-\mathrm{b}_{\mathrm{n}}\right|<\delta$ for $n$ $\leq m$, we obtain

$$
p(\Phi(b)-\Phi(a)) \leq \delta \sum_{n=0}^{m} p\left(x_{m}\right)+\epsilon \sum_{n=m+1}^{\infty}\left|b_{n}-a_{n}\right| .
$$

Using the definition of $\delta$ and inequalities $\|a\|_{1} \leq 1,\|b\|_{1} \leq 1$, we see that $p(\varphi(b)-\varphi(a)) \leq 3 \varepsilon$. Since $a, p$ and $\varepsilon$ are arbitrary, the map $\varphi$ is continuous. Hence $K$ is compact and metrizable as a continuous image of a compact metrizable space. Obviously $K$ is convex and balanced. Hence $K$ is a Banach disk (any compact disk is a Banach disk). Let us show that $E$ is dense in $X_{K^{*}}$. Take $u \in X_{K^{*}}$. Then there is $a \in \ell_{1}$ such that $u=\sum_{k=0}^{\infty} a_{k} x_{k}$. Clearly, for any $n \in \mathbb{Z}_{+}, u_{n}=\sum_{k=0}^{n} a_{k} x_{k} \in E$.

Let $\|\cdot\|$ be the norm of the Banach space $X_{K}$. Then for any $n \in \mathbb{Z}_{+}$,

$$
\left\|u-u_{n}\right\|=\left\|\sum_{k=n+1}^{\infty} a_{k} x_{k}\right\| \leq \sum_{k=n+1}^{\infty} a_{k} \mid \rightarrow 0 \text { as } n \rightarrow \infty .
$$

Hence $E$ is dense in $X_{K}$ and therefore $X_{K}$ is separable and $K$ is a Banach s-disk.

Lemma 3.4. Let $X$ be a separable metrizable topological vector space and $\left\{f_{n}\right\}_{n \in \mathbb{Z}_{+}}$be a linearly independent sequence in $X$. Then there exist sequences $\left\{x_{n}\right\}_{n \in \mathbb{Z}_{+}}$in $X$ and $\left\{\alpha_{k, j}\right\}_{k, j \in \mathbb{Z}_{+} j<k}$, in $\mathbb{K}$ such that span $\left\{x_{k}\right.$ : $\left.k \in \mathbb{Z}_{+}\right\}$is dense in $X, g_{n}\left(x_{k}\right)=0$ for $n \neq k$ and $g_{n}\left(x_{k}\right) \neq 0$ for $n \in \mathbb{Z}_{+}$, where $g_{n}=f_{n}+\sum_{j<n} \alpha_{n, j} f_{j}$.

Proof. Let $\left\{U_{n}\right\}_{n \in \mathbb{Z}_{+}}$be a base of topology of $X$. First, we construct inductively sequences $\left\{\alpha_{k, j}\right\}_{k, j \in \mathbb{Z}_{+}, j<k}$ in $\mathbb{K}$ and $\left\{y_{n}\right\}_{n \in \mathbb{Z}_{+}}$in $X$ such that for any $k \in \mathbb{Z}_{+}$,

$$
\begin{aligned}
& \text { (b1) } y_{k} \in U_{k} \text {; } \\
& \text { (b2) } g_{k}\left(y_{k}\right) \neq 0 \text {, where } g_{k}=f_{k}+\sum_{j<k} \alpha_{\mathrm{k}, j} f_{j} \text {; }
\end{aligned}
$$

\section{(b3) $g_{k}\left(y_{k}\right)=0$ if $m<k$.}

Let $g_{0}=f_{0}$. Since $f_{0} \neq 0$, there is $y_{0} \in U_{0}$ such that $f_{0}\left(y_{0}\right)=g_{0}\left(y_{0}\right) \neq 0$. This provides us with the base of induction. Assume now that $n \in \mathbb{N}$ and $y_{k}$, $\alpha_{k, j}$ with $j<k<n$ satisfying (b1-b3) are already constructed. According to (b2) and (b3), we can find $\alpha_{n}, 0, \ldots, \alpha_{n, n-1} \in \mathbb{K}$ such that $g_{n}\left(y_{m}\right)=0$ for $m$ $<n$, where $g_{n}=f_{n}+\sum_{j<n} \alpha_{n, j} f_{j}$. Next since $f_{j}$ are linearly independent, $g_{n}$ $\neq 0$ and therefore there is $y_{n} \in U_{n}$ such that $g_{n}\left(y_{n}\right) \neq 0$. This concludes the description of the inductive procedure of constructing sequences $\{k j\}_{k, j \in \mathbb{Z}} j<$ and $\left\{y_{n}\right\}_{n \in \mathbb{Z}_{+}}$satisfying (b1-b3) for each $\mathrm{k} \in \mathbb{Z}_{+}$.

Using (b2) and (b3), one can easily demonstrate that there is a sequence $\left\{\beta_{k, j}\right\}_{k, j \in \mathbb{Z}_{+}, j<k}$ in $\mathbb{K}$ such that $g_{n}\left(x_{k}\right)=0$ for $k \neq m$, $x_{k}=y_{k}+\sum_{j<k} \beta_{k, j} y_{j}$. From (b2) and (b3) it also follows that $g_{n}\left(x_{n}\right) 0$ for each $n \in \mathbb{Z}_{+}$. It remains to notice that according to (b1), $\left\{y_{n}: n \in \mathbb{Z}_{+}\right\}$is dense in $X$. Since $\left\{\mathrm{y}_{\mathrm{n}}: \mathrm{n} \in \mathbb{Z}_{+}\right\} \subseteq \operatorname{span}\left\{\mathrm{y}_{\mathrm{n}}: \mathrm{n} \in \mathbb{Z}_{+}\right\}=\operatorname{span}\left\{\mathrm{x}_{\mathrm{n}}: \mathrm{n} \in \mathbb{Z}_{+}\right\}$, we see that $\operatorname{span}\left\{\mathrm{x}_{\mathrm{n}}: \mathrm{n} \in \mathbb{Z}_{+}\right\}$is dense in $X$.

Lemma 3.5. Let $X \in \mathfrak{M}$. Then there exist sequences $\left\{x_{n}\right\}_{n \in \mathbb{Z}_{+}}$and $\left\{f_{k}\right\}_{k \in \mathbb{Z}_{+}}$in $X$ and $X^{\prime}$ rspectively, such that

(3.5.1) $\left\{x_{n}\right\}_{n \in \mathbb{Z}_{+}}$is an $\ell_{1}$-sequence in $X$;

(3.5.2) the space E=span $\left\{x_{n}: n \in \mathbb{Z}_{+}\right\}$is dense in $X$;

(3.5.3) $f_{k}\left(x_{n}\right)=0$ if $k \neq n$ and $f_{k}\left(x_{k}\right) \neq 0$ for each $k \in \mathbb{Z}_{+}$;

(3.5.4) $\left\{f_{k}: k \in \mathbb{Z}_{+}\right\}$is uniformly equicontinuous.

Moreover, $\left\{f_{k}\right\}$ can be chosen from the linear span of any linearly independent uniformly equicontinuous sequence in $X^{\prime}$.

Proof. According to Lemma 3.3, there exists a Banach s-disk $K$ in $X$ such that $X_{K}$ is dense in $X$. By Corollary 3.2 , there is a linearly independent sequence $\left\{g_{n}\right\}_{n \in \mathbb{N}_{+}}$in $X^{\prime}$ such that $\left\{g_{n}: n \in \mathbb{N}\right\}$ is uniformly equicontinuous. Since $X_{K}$ is dense in $X$, the functionals $\left.g_{n}\right|_{X_{K}}$ on $X_{K}$ are linearly independent. Applying Lemma 3.4 to the sequence $\left\{\left.g_{n}\right|_{X_{K}}\right\}$, we find that there exist sequences $\left\{\mathrm{y}_{n}\right\}_{n \in \mathbb{Z}_{+}}$in $X_{K}$ and $\left\{\alpha_{k, j}\right\}_{k, j \in \mathbb{Z}_{+}, j<k}$ in $\mathbb{K}$ such that $E=\operatorname{span}\left\{y_{k}: k \in \mathbb{Z}_{+}\right\}$is dense in $X_{K}, h_{n}\left(y_{k}\right)=0$ for $n \neq k$ and $h_{n}\left(y_{n}\right) \neq 0$ for $\mathrm{n} \in \mathbb{Z}_{+}$, where $h_{n}=g_{n}+\sum_{j<n} \alpha_{n, j} g_{j}$. Let q be the norm of the Banach space $X_{K^{*}}$ Consider $f_{n}=c_{n} h_{n}$, where $c_{n}=\left(1+\sum_{j<n}\left|\alpha_{n, j}\right|\right)^{-1}$. Since $\left\{g_{n}: n \in \mathbb{N}\right\}$ is uniformly equicontinuous and $h_{n}=g_{n}+\sum_{j<n} \alpha_{n, j} g_{j}$, we immediately see that $\left\{f_{n}: n \in \mathbb{N}\right\}$ is uniformly equicontinuous. Next, let $x_{n}=b_{n} y_{n}$, where $b_{n}=2^{-n} q\left(x_{n}\right)^{-1}$. Since $x_{n}$ converges to 0 in the Banach space $X_{K},\left\{x_{n}\right\}_{n \in \mathbb{N}}$ is an $\ell_{1}$-sequence in $X_{K}$. Since the topology of $X_{K}$ is stronger than the one inherited from $X,\left\{x_{n}\right\}_{n \in \mathbb{N}}$ is an $\ell_{1}$-sequence in $X$. Since $\operatorname{span}\left\{x_{n}: n \in \mathbb{Z}_{+}\right\}=\operatorname{span}\left\{y_{n}: n \in \mathbb{Z}_{+}\right\}$is dense in $X_{K}$, it is also dense in $X_{K}$ in the topology inherited from $X$. Since $X_{K}$ is dense in $X$, span $\left\{x_{n}: n \in \mathbb{Z}_{+}\right\}$is dense in $X$. Finally since $f_{n}\left(x_{k}\right)=c_{n} b_{k} h_{n}\left(y_{k}\right)$, we see that $f_{n}\left(x_{k}\right)=0$ if $n \neq k$ and $f_{n}\left(x_{n}\right) \neq 0$ for any $n \in \mathbb{Z}_{+}$. Thus conditions (3.5.1-3.5.4) are satisfied.

Lemma 3.6. Let $X$ be a locally convex space, whose topology is not weak and $Y$ be a locally convex space admitting an $\ell_{1}$-sequence with dense span. Then there is $T \in L(Y, X)$ such that $T(X)$ is dense in $Y$. 
Proof. Let $\left\{y_{n}\right\}_{n \in \mathbb{Z}_{+}}$be an $\ell_{1}$-sequence in $Y$ with dense span. By Corollary 3.2, there is a uniformly equicontinuous sequence $\left\{f_{n}\right\}_{n \in \mathbb{N}_{+}}$ in $\mathrm{X}^{\prime}$ such that $\varphi \subseteq\left\{\left\{f_{n}(x)\right\}_{n \in \mathbb{Z}_{+}}: x \in X\right\}$.. Consider the linear operator $T$ : $X \rightarrow Y$ defined by the formula $T x=\sum_{n=0}^{\infty} 2^{-n} f_{n}(x) y_{n}$.Since $\left\{f_{n}\right\}$ is uniformly equicontinuous, there is a continuous seminorm $p$ on $X$ such that $\left|f_{n}(x)\right|$ $\leq p(x)$ for any $x \in X$ and $n \in \mathbb{Z}_{+}$. Since $\left\{y_{n}\right\}$ is an $\ell_{1}$-sequence, Lemma 3.3 implies that the closed convex balanced hull $Q$ of $\left\{y_{n}: n \in \mathbb{Z}_{+}\right\}$is compact and metrizable. Let $q$ be the Minkowskii functional of $Q$ (=the norm on $\left.Y_{Q}\right)$. It is easy to see that $q(T x) \leq p(x)$ for each $x \in X$. Hence $T$ is continuous as an operator from $X$ to the Banach space $Y_{Q}$. Since the latter carries the topology stronger than the one inherited from $Y$, $T \in L(X, Y)$. Next, the inclusion $\varphi \subseteq\left\{\left\{f_{n}(x)\right\}_{n \in \mathbb{Z}_{+}}: x \in x\right\}$, implies that $T(X)$ contains the linear span of $\left\{y_{n}: n \in \mathbb{Z}_{+}\right\}$, which is dense in $Y$. Thus $T$ has dense range.

Lemma 3.7. Let $X$ be an infinite dimensional locally convex space and assume that there exist $\ell_{1}$-sequences with dense span in both $X$ and $X_{\beta}^{\prime}$. Then there exist sequences $\left\{x_{n}\right\}_{n \in \mathbb{N}_{+}}$and $\left\{f_{k}\right\}_{k \in \mathbb{Z}_{+}}$in $X$ and $X^{\prime}$ respectively, such that

(3.7.1) $\left\{x_{n}\right\}_{n \in \mathbb{Z}_{+}}$is an $\ell_{1}$-sequence in $X$ and $\left\{f_{n}\right\}_{n \in \mathbb{Z}_{+}}$is an $\ell_{1}^{-}$ sequence in $X_{\beta}^{\prime}$;

(3.7.2) E=span $\left\{x_{n}: n \in \mathbb{Z}_{+}\right\}$is dense in $X$ and $F=\operatorname{span}\left\{f_{n}: n \in \mathbb{Z}_{+}\right\}$is dense in $X_{\beta}^{\prime}$;

\section{(3.7.3) $f_{k}\left(x_{n}\right)=0$ if $k \neq n$ and $f_{k}\left(x_{k}\right) \neq 0$ for each $k \in \mathbb{Z}_{+}$.}

If the original $\ell_{1}$-sequence in $X_{\beta}^{\prime}$ is uniformly equicontinuous, than we can also ensure that (3.7.4) $\left\{f_{n}: n \in \mathbb{Z}_{+}\right\}$is uniformly equicontinuous.

Proof. By Lemma 3.3, there exists a Banach $s$-disk $K$ in $X$ such that $X_{K}$ is dense in $X$. Fix an $\ell_{1}$-sequence $\left\{g_{n}\right\}_{n \in \mathbb{N}}$ in $X_{\beta}^{\prime}$ with dense span. Since any sequence of elements of a linear space with infinite dimensional span has a linearly independent subsequence with the same span, we, passing to a subsequence, if necessary, can assume that $g_{n}$ are linearly independent. Since $X_{K}$ is dense in $X$, the functionals $\left.g_{n}\right|_{X_{K}}$ on $X_{K}$ are linearly independent. Let $D$ be the closed absolutely convex hull of the set $\left\{g_{n}: n \in \mathbb{Z}_{+}\right\}$in $X_{\beta}^{\prime}$. By Lemma 3.3, $D$ is a Banach disk in $X_{\beta}^{\prime}$. Applying Lemma 3.4 to the sequence $\left\{\left.g_{n}\right|_{X_{K}}\right\}$, we find that there exist sequences $\left\{y_{n}\right\}_{n \in \mathbb{Z}_{+}}$in $X_{K}$ and $\left\{\alpha_{k, j}\right\}_{k, j \in \mathbb{Z}_{+}, j<k}$ in $\mathbb{K}$ such that $E=\operatorname{span}\left\{y_{k}: k \in \mathbb{Z}_{+}\right\}$is dense in $X_{K}, h_{n}\left(y_{k}\right)=0$ for $n \neq k$ and $h_{n}\left(y_{n}\right)$ $\neq 0$ for $n \in \mathbb{Z}_{+}$, where $h_{n}=g_{n}+\sum_{j<n} \alpha_{n, j} g_{j}$. Let $q$ be the norm of the Banach space $X_{K}$ and $p$ be the norm of the Banach space $X_{D}^{\prime}$. Consider $f_{\mathrm{n}}=c_{n} h_{n}$, where $c_{n}=2^{-n}\left(1+\sum_{j<n}\left|\alpha_{n, j}\right|\right)^{-1}$. Since $p\left(g_{\mathrm{n}}\right) \leq 1$ for each $n \in \mathbb{Z}_{+}$

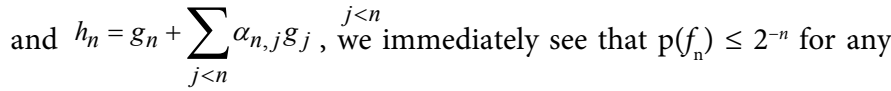
$n \in \mathbb{Z}_{+}$. Hence $\left\{f_{n}\right\}_{n \in \mathbb{Z}_{+}}$is an $\ell_{1}$-sequence in the Banach space $X^{\prime}{ }_{D^{\circ}}$ Since the topology of $X^{\prime}{ }_{D}$ is stronger than the one inherited from $X_{\beta^{\prime}}^{\prime}$, $\left\{f_{n}\right\}_{n \in \mathbb{Z}_{+}}$is an $\ell_{1}$-sequence in $X_{\beta}^{\prime}$. Similarly, $\left\{f_{\mathrm{n}}: \mathrm{n} \in \mathbb{Z}_{+}\right\}$is uniformly equicontinuous if $\left\{g_{n}: n \in \mathbb{Z}_{+}\right\}$is. Since the sequences $\left\{g_{n}\right\}_{n \in \mathbb{Z}_{+}}$and $\left\{f_{n}\right\}_{n \in \mathbb{Z}_{+}}$have the same spans, the span of $\left\{f_{n}: n \in \mathbb{Z}_{+}\right\}$is dense in $X^{\prime}$. Next, let $x_{n}=b_{n} y_{n}$, where $b_{n}=2^{-n} q\left(x_{n}\right)^{-1}$. Since $x_{n}$ converges to 0 in the
Banach space $X_{K},\left\{x_{n}\right\}_{n \in \mathbb{N}}$ is an $\ell_{1}$-sequence in $X_{K}$. Since the topology of $X_{K}$ is stronger than the one inherited from $X,\left\{x_{n}\right\}_{n \in \mathbb{N}}$ is an $\ell_{1}$ sequence in $X$. Since $\operatorname{span}\left\{x_{n}: n \in \mathbb{Z}_{+}\right\}=\operatorname{span}\left\{y_{n}: n \in \mathbb{Z}_{+}\right\}$is dense in $X_{K^{\prime}}$ it is also dense in $X_{K}$ in the topology inherited from $X$. Since $X_{K}$ is dense in $X$, span $\left\{x_{n}: n \in \mathbb{Z}_{+}\right\}$is dense in $X$. Finally since $f_{n}\left(x_{k}\right)=c_{n} b_{k} h_{n}\left(y_{k}\right)$, we see that $f_{n}\left(x_{k}\right)=0$ if $n \neq k$ and $f_{n}\left(x_{n}\right) \neq 0$ for any $n \in \mathbb{Z}_{+}$. Thus conditions (3.7.1-3.5.3) (and (3.5.4) if $\left\{g_{n}: n \in \mathbb{Z}_{+}\right\}$is uniformly equicontinuous) are satisfied.

Lemma 3.8. The class $\mathfrak{M}$ is stable under finite or countable products. Moreover, if $X \in \mathfrak{M}$, then $X \times \mathbb{K} \in \mathfrak{M}$.

Proof. It is clear that if the topology of one of the locally convex spaces $X_{j}$ is not weak, then so is the topology of their product $\prod x_{j}$. Thus the only thing we have to worry about is the existence of an $\ell_{1}$-sequence with dense span. Let J be a finite or countable infinite set, $X_{j} \in \mathfrak{M}$ for each $j \in J$ and $x=\prod_{j \in J} x_{j}$. Let for any $j \in J,\left\{x_{j, n}\right\}_{n \in \mathbb{Z}_{+}}$be an $\ell_{1}$-sequence in $X j$ with dense span. For $(j, n) \in J \times \mathbb{Z}_{+}$let $u_{j, n} \in X$ be such that $j^{\text {th }}$ component of $u_{j, n}$ is $x_{j, n}$, while all other components are 0 . Clearly $\left\{u_{j, n}\right.$ : $\left.j \in J, n \in \mathbb{Z}_{+}\right\}$is a countable subset of $X$. It is easy to see that enumerating this subset by elements of $\mathbb{Z}_{+}$, we get an $\ell_{1}$-sequence in $X$ with dense span. The proof of the second part of the lemma is even easier: one have just to add one vector $(0,1)$ to an $\ell_{1}$-sequence with dense span in $X=X \times$ $\{0\}$, to obtain an $\ell_{1}$-sequence with dense span in $X \times \mathbb{K}$.

\section{Proof of Theorem 1.3}

Let $X \in \mathfrak{M}$. By Lemma 3.5, there exist sequences $\left\{x_{n}\right\}_{n \in \mathbb{Z}_{+}}$and $\left\{f_{k}\right\}_{k \in \mathbb{Z}_{+}}$in $X$ and $X^{\prime}$ respectively satisfying (3.5.1-3.5.4). Uniform equicontinuity of $\left\{f_{n}\right\}$ is equivalent to the existence of a continuous seminorm $p$ on $X$ such that each $\left|f_{n}\right|$ is bounded by 1 on the unit ball $\{x \in X: p(x) \leq 1\}$ of $p$. Since $\left\{x_{n}\right\}$ is an $\ell_{1}$-sequence in $X$, Lemma 3.3, absolutely convex closed hull $K$ of $\left\{x_{n}: n \in \mathbb{Z}_{+}\right\}$is compact and is a Banach disk in $X$. Let $q$ be the norm of the Banach space $X_{K^{*}}$. Then $q\left(x_{n}\right)$ $\leq 1$ for each $n \in \mathbb{Z}_{+}$. Let $\mathrm{c}=\sup \{p(x): x \in K\}$. Compactness of $K$ implies that $\mathrm{c}$ is finite. Clearly $c>0$ and $p(x) \leq c q(x)$ for any $x \in Y$.

Lemma 4.1. Let $\alpha, \beta: \mathbb{Z}_{+} \rightarrow \mathbb{Z}_{+}$be any maps and $a=\left\{a_{n}\right\}_{n \in \mathbb{Z}_{+}} \in \ell_{1}$. Then the formula

$$
T x=\sum_{n \in \mathbb{Z}_{+}} a_{n} f_{\alpha(n)}(x) x_{\beta(n)}
$$

defines a linear operator on $X$. Moreover, the series $\sum_{n=0}^{\infty} \frac{z^{n}}{n !} T^{n} x=e^{z T} x$
nverges in $X$ for any $x \in X$ anid $z \in \mathbb{K}$ and

$q(T x) \leq\|a\| p(x), \quad q\left(e^{z T} x-x\right) \leq c^{-1} e^{c|| a \||| z \mid} p(x)$ for each $x \in X$ and $z \in \mathbb{K}$, where $\|a\|$ is the $\ell_{1}$-norm of $a$.

Proof. Condition (3.5.4) implies that the sequence $\left\{f_{\alpha(n)}(x)\right\}$ is bounded for any $x \in X$. Since $\left\{x_{n}\right\}$ is an $\ell_{1}$-sequence and $a \in \ell_{1}$, we see that the series in (4.1) converges for any $x \in X$ and therefore defines a linear operator on $X$. Moreover, if $p(x) \leq 1$, then $\left|f_{k}(x)\right| \leq 1$ for each $k$ $\in \mathbb{Z}_{+}$and since $q\left(x_{m}\right) \leq 1$ for $m \in \mathbb{Z}$, formula (4.1) implies that $q(T x) \leq$ $\|a\|$. Hence $q(T x) \leq\|a\| p(x)$ for each $x \in X$. Then $q\left(T^{2} x\right) \leq\|a\| p(T x) \leq$ $c\|a\| q(T x) \leq c\|a\|^{2} p(x)$. Iterating this argument, we see that

$q\left(T^{n} x\right) \leq c^{n-1}\|a\|^{n} p(x)$ for each $n \in \mathbb{N}$.

It follows that for any $x \in X$, the series $\sum_{n=1}^{\infty} \frac{z^{n}}{\mathrm{k} !} T^{n} x$ is absolutely convergent in the Banach space $X_{K}$ (and therefore in $X$ ) for any $z \in \mathbb{K}$. Naturally we denote its sum as $e^{z T} x$. Moreover, using the above display, 
we obtain

$$
q\left(e^{z T} x-x\right)=q\left(\sum_{n=1}^{\infty} \frac{z^{n}}{n !} T^{n} x\right) \leq c^{-1} p(x) \sum_{n=1}^{\infty} \frac{\left(c\|a|\| z|)^{n}\right.}{n !}<c^{-1} e^{c|| a \||| z \mid} p(x) .
$$

Thus (4.2) is satisfied.

Now fix a bijection $\varphi: \mathbb{Z}_{+}^{k} \rightarrow \mathbb{Z}_{+}$. By symbol $e_{j}$ we denote the element of $\mathbb{Z}_{+}^{k}$ defined by $\left(e_{j}\right)_{l}=\delta_{j, p}$, where $\delta_{j, l}$ is the Kronecker delta. For $n \in \mathbb{Z}_{+}^{k}$, we also write $|n|=n_{1}+\ldots+n_{k}$. For each $m \in \mathbb{Z}_{+}$let

$$
\varepsilon_{m}=\min _{\substack{n \in \mathbb{Z}_{+}^{k} \\|n|=m+1}}\left|f_{\varphi(n)}\left(x_{\varphi(n)}\right)\right| .
$$

According to (3.5.1), (3.5.3) and (3.5.4), $\left\{\varepsilon_{m}\right\}$ is a bounded sequence positive numbers. Next, we pick any sequence $\left\{\alpha_{m}\right\}_{m \in \mathbb{Z}_{+}}$of positive numbers such that

$$
\alpha_{m+1} \geq 2^{m} \alpha_{m} \varepsilon_{m}^{-1} \text { for any } m \in \mathbb{Z}_{+}
$$

and consider the operators $A_{j} \in L(X)$ for $1 \leq j \leq k$ defined by the formula

$$
A_{j} x=\sum_{n \in \mathbb{Z}_{+}^{k}} \frac{\alpha_{|n|} f_{\varphi\left(n+e_{j}\right)}(x)}{\alpha_{|n|+1} f_{\varphi\left(n+e_{j}\right)}\left(x_{\varphi\left(n+e_{j}\right)}\right)} x_{\varphi(n)} .
$$

From the estimates (4.3) it follows that the series defining $A_{j}$ can be written as

$$
A_{j} x=\sum_{n \in \mathbb{Z}_{+}^{k}} c_{j, n} f_{\phi\left(n+e_{j}\right)}(x) x_{\phi(n)} \text { with } 0<\left|\mathrm{c}_{\mathrm{j}, \mathrm{n}}\right|<2^{-|\mathrm{n}|}
$$

Clearly $A_{j}$ have shape (4.1) with $\|a\| \leq C=\sum_{n \in \mathbb{Z}_{+}^{k}} 2^{-|n|}$. By Lemma 4.1, $A_{j}$ are linear operators on $X$ satisfying $q(T x) \leq C p(x)$ for any $x$

$\in X$. Hence $A_{j}$ are continuous as operators from $X$ to the Banach space $X_{K}$. Since $X_{K}$ carries a stronger topology than the one inherited from $X, A_{j} \in L(X)$ for $1 \leq j \leq k$. Next, using the definition of $A_{\text {; }}$, it is easy to verify that $A_{1} A_{x_{n}}=A_{t_{1}} x_{n}$ for any $1 \leq j<l \leq k$ and $n \in \mathbb{Z}_{+}$. Indeed, for any $n \in \mathbb{Z}_{+}$, there is a unique $m \in \mathbb{Z}_{+}^{k}$ such that $n=\varphi(m)$. If either $m_{j}=0$ or $m_{l}=0$, from the definition of $A_{j}$ and $A_{l}$ it follows that $A_{j} A_{l} x_{n}=A_{l} A_{j} x_{n}=0$.If $m_{j} \geq 1$ and $m_{l} \geq 1$ from the same definition we obtain $A_{j} A_{l} x_{n}=A_{l} A_{j} x_{n}=\frac{\alpha_{|m|-2}}{\alpha_{|m|}} x_{\varphi\left(m-e_{j}-e_{l}\right)}$.From (3.5.2) it follows now that the operators $A_{1}, \ldots, A_{n}$ are pairwise commuting. Next, for $z \in \mathbb{K}^{\mathrm{k}}$, the operator $\langle z, A\rangle$ has shape (4.1) with $\|a\| \leq C\|z\|$, where $\|z\|$ is the $\ell_{1}$ norm of $z$. By Lemma 4.1, the series $\sum_{n=0}^{\infty} \frac{1}{n !}\langle z, A\rangle^{n}$ converges pointwisely to a linear operator,$\langle z, A\rangle$ and

$$
q\left(e^{\langle z, A\rangle} x-x\right) \leq c^{-1} e^{c C\|z\|} p(x) \text { for any } x \in X \text { and } z \in \mathbb{K}^{\mathrm{k}} .
$$

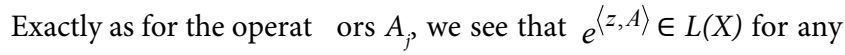
$z \in \mathbb{K}^{\mathrm{k}}$. From (4.4), if $z_{n} \rightarrow z$ in $\mathbb{K}^{k}$, then $e^{\left\langle z_{n}, A\right\rangle} x \rightarrow e^{\langle z, A\rangle} x$ uniformly on $\{x \in X: p(x)<1\}$. By Proposition 2.8, $\left\{e^{\langle z, A\rangle}\right\}_{z \in \mathbb{K}^{k}}$ is a uniformly continuous group and the map $z \mapsto e^{\langle z, A\rangle} x$ is holomorphic for any $x \in X$ provided $\mathbb{K}=\mathbb{C}$. The proof will be complete if we show that the group $\left\{e^{\langle z, A\rangle}\right\}_{z \in \mathbb{K}^{k}}$ is hereditarily hypercyclic. To this end consider the restrictions $B_{j}$ of $A_{j}$ to $X_{K}$ as bounded linear operators on the
Banach space $X_{K^{*}}$. Then $B_{j}$ commute with each other as the restrictions of commuting operators. Let us show that the operator group $\left\{e^{\langle z, A\rangle}\right\}_{z \in \mathbb{K}^{k}}$ on $X_{K}$ is hereditarily hypercyclic. By Corollary 2.14, it suffices to verify that $B=\left(B_{1}, \ldots, B_{k}\right)$ is a $\mathrm{EBS}_{k}$-tuple. We already know that $B_{j}$ are pairwise commuting. From the definition of $B_{j}$ it is easy to see that $\operatorname{ker} B^{m}{ }_{j}$ contains $E_{j, m}=\operatorname{span}\left\{x_{\varphi(n)}: n \in \mathbb{Z}_{+}^{k}, n_{j} \leq m-1\right\}$.Using this fact it is easy to see that for any $m \in \mathbb{N}^{k}$, the set $\kappa(m, B)$ defined by (2.2) contains

$$
E_{m}=\bigcap_{j=1}^{k} E_{j, m_{j}}=\operatorname{span}\left\{x_{\varphi(n)}: n \in \mathbb{Z}_{+}^{k}, n_{j} \leq m_{j}-1,1 \leq j \leq k\right\} .
$$

Hence the space $\operatorname{ker}^{\dagger} B$ defined in (2.2) contains $\bigcup_{m \in \mathbb{N}^{k}} E_{m}=\operatorname{span}\left\{x_{n}: n \in \mathbb{Z}_{+}\right\}$. By Lemma 3.3, $\operatorname{ker}^{\dagger}(B)$ is dense in $X_{K^{*}}$ Hence $B$ is an $\mathrm{EBS}_{k}$-tuple. Thus according to Corollary 2.14, $\left\{e^{\langle z, A\rangle}\right\}_{z \in \mathbb{K}^{k}}$ is hereditarily hypercyclic. Since $X_{K}$ is dense in $X$ and carries a topology stronger than the one inherited from $X,\left\{e^{\langle z, A\rangle}\right\}_{z \in \mathbb{K}^{k}}$ is hereditarily hypercyclic. By Proposition 1.1, $\left\{e^{\langle z, A\rangle}\right\}_{z \in \mathbb{K}^{k}}$ is mixing.

\section{Theorem 1.18: proof and applications}

Proof of Theorem 1.18. Assume that $T$ is non-mixing. Then we can choose non-empty open subsets $U$ and $V$ of $X$ and a strictly increasing sequence $\left\{n_{k}\right\}_{k \in \mathbb{Z}_{+}}$of positive integers such that $T^{n_{k}}(U) \cap V=\varnothing$ for any $k \in \mathbb{Z}_{+}$. Let $\Sigma$ be the set of $(x, y) \in X \times X$ for which there exists a sequence $\left\{x_{k}\right\}_{n \in \mathbb{Z}_{+}}$of elements of $X$ such that $x_{k} \rightarrow x$ and $T^{n_{k}} x_{k} \rightarrow y$. According to Theorem 2.12, $\Sigma$ contains $\Lambda(T) \times \Lambda(T)$. Since $\Lambda(T)$ is dense in $X$, we see that $\Sigma$ is dense in $X \times X$. Hence $\Sigma$ intersects $U \times V$, which is impossible since $T^{n_{k}}(U) \cap V=\varnothing$ for any $k \in \mathbb{Z}_{+}$. This contradiction shows that $T$ is mixing. If $X$ is Baire separable and metrizable, then by Proposition 1.1, $T$ is hereditarily hypercyclic.

\section{Extensions of the Salas theorem}

It is easy to see that $\operatorname{ker}^{\dagger} T \subseteq \Lambda(I+T)$ for any linear operator $T$. Thus Theorem 1.18 implies the following corollary.

Corollary 5.1. Let $T$ be an extended backward shift on a topological vector space $X$. Then $I+T$ is mixing. If additionally $X$ is Baire, separable and metrizable, then $I+T$ is hereditarily hypercyclic.

Recall that a backward weighted shift on $\ell_{\mathrm{p}}=\ell_{\mathrm{p}}\left(\mathbb{Z}_{+}\right)$for $1 \leq p<\infty$ or $c_{0}=c_{0}\left(\mathbb{Z}_{+}\right)$is the operator $T$ acting on the canonical basis $\left\{e_{n}\right\}_{n=0}^{\infty}$ as follows: $T e_{0}=0$ and $T e_{n}=w_{n} e_{n-1}$ for $n \geq 1$, where $w=\left\{w_{n}\right\}_{n \in \mathbb{N}}$ is a bounded sequence of non-zero numbers in $\mathbb{K}$. Clearly any backward weighted shift is a generalized backward shift and therefore is an extended backward shift. Hence Corollary 5.1 contains the Salas theorem on hypercyclicity of the operators $I+T$ with $T$ being a backward weighted shift as a particular case. It is also easy to see that if $X$ is a topological vector space and $T \in L(X)$ is surjective, then $\operatorname{ker}^{\dagger} T=k e r^{\star} T$. Thus we obtain the following corollary.

Corollary 5.2. Let $X$ be a topological vector space and $T \in L(X)$ be such that $T(X)=X$ and $k^{*} r^{*} T$ is dense in $X$. Then $I+T$ is mixing. If additionally $X$ is Baire, separable and metrizable, then $I+T$ is hereditarily hypercyclic.

We can further generalize Corollary 5.1 by means of the following observation. 
Lemma 5.3. Let $X$ be a topological vector space, $k \in \mathbb{N}, T \in L(X)$ and $\left\{a_{j}\right\}_{j \in \mathbb{N}}$ be a sequence in $\mathbb{K}$ such that the series $\sum_{j=1}^{\infty} a_{j} T^{k+j}$ of operators converges pointwise to a continuous linear operator $S$ on $X$. Then $k e r^{\dagger} T^{k}$ $\subseteq \operatorname{ker}^{\dagger}\left(T^{k}+S\right)$.

Proof. In order to prove the inclusion $\operatorname{ker}^{\dagger} T^{k} \subseteq \operatorname{ker}^{\dagger}\left(T^{k}+S\right)$, it is enough to show that $k e r T^{k n} \cap T^{k n}(X) \subseteq k e r^{\dagger}\left(T^{k}+S\right)$ for any $n \in \mathbb{N}$. Thus pick $n \in \mathbb{N}$ and let $x \in \operatorname{ker} T^{k n} \cap T^{k n}(X)$. Then $T^{k n} x=0$ and there is $y$ $\in X$ such that $T^{k n} y=x$. For $1 \leq j \leq 2 k n$ denote $h_{j}=T^{2 k n-j} y$. It is easy to see that $T h_{1}=0, T h_{j}=h_{j-1}$ for $2 \leq j \leq 2 k n$ and $h_{k n}=x$. Consider the backward shift $B$ on the space $\mathbb{K}^{2 k n}$, which acts on the canonical basic vectors by the same rule: $B e_{1}=0$ and $B e_{j}=e_{j-1}$ for $2 \leq j \leq 2 \mathrm{kn}$. Let $Y=\operatorname{span}\left\{h_{j}: 1 \leq j \leq 2 k n\right\}$ and consider the surjective linear operator $J: \mathbb{K}^{2 k n} \rightarrow Y$ defined by $J e_{j}=h_{j}$ for $1 \leq j \leq 2 k n$. It is easy to see that $Y$ is $T$-invariant and $T_{Y} J=J B$, where $T_{Y} \in L(Y)$ is the restriction of $T$ to $Y$. Since $\mathrm{B}^{2 k n}=0$, we see that $T_{Y}^{2 k n}=0$ and therefore the restriction $R \in L(Y)$ of $T^{k}+S$ to $Y$ is given by the formula $R=p\left(T_{Y}\right)$, where $P$ is the polynomial defined by $p(z)=z^{k}+\sum_{j=1}^{2 k n-k-1} a_{j} z^{k+j}$. Let $E=\operatorname{span}\left\{e_{j}\right.$ : $1 \leq j \leq k n$ \}. Considering the matrix of the operator $p(B)$, it is easy to see that $e_{k n} \in E=\operatorname{ker} p(B)^{n}=p(B)^{n}(\mathbb{K})$. Using the intertwining relation $T_{Y} J=J B$, we see that $x=J\left(e_{k n}\right) \in J(E) \subseteq \operatorname{ker} p\left(T_{Y}\right)^{n} \cap p\left(T_{Y}\right)^{n}(Y)$. Since $R=p\left(T_{Y}\right)$ is the restriction to $Y$ of the operator $T^{k}+S$, we see that $x \in \operatorname{ker} p\left(T^{k}+S\right)^{n} \cap\left(T^{k}+S\right)^{n}(X) \subseteq \operatorname{ker}^{\dagger}\left(T^{k}+S\right)$.

The following result is an immediate consequence of Lemma 5.3 and Corollary 5.1.

Corollary 5.4 Let $X$ be a topological vector space, $k \in \mathbb{N}, T \in L(X)$ and $\left\{a_{j}\right\}_{j \in \mathbb{N}}$ be a sequence in $\mathbb{K}$ such that the series $\sum_{j=1}^{\infty} a_{j} T^{k+j}$ of operators converges pointwise to a continuous linear operator $S$ on X. Assume also that $T^{k}$ is an extended backward shift. Then the operator $I+T^{k}+S$ is mixing. If additionally $X$ is Baire separable and metrizable, then $I+T^{k}+S$ is hereditarily hypercyclic.

It is straightforward to verify that any power of a generalized weighted shift is an extended backward shift. Thus the above corollary implies the next observation.

Corollary 5.5 Let $X$ be a topological vector space, $T \in L(X)$ be a generalized backward shift and $\left\{a_{j}\right\}_{j \in \mathbb{Z}_{+}}$be a sequence in $\mathbb{K}$ such that $a_{0}=1$, there is $n \in \mathbb{N}$ for which $a_{n} \neq 0$ and the series $\sum_{j=0}^{\infty} a_{j} T^{j}$ converges pointwise to a continuous linear operator $S$ on $X$. Then $S$ is mixing. If additionally $X$ is Baire separable and metrizable, then $S$ is hereditarily hypercyclic.

\section{An extension of the Hilden-Wallen theorem}

Lemma 5.6 Let $X$ be a Baire topological space, $Y$ be a second countable topological space and $\left\{T_{n}\right\}_{n \in \mathbb{Z}}$ be a sequence of continuous maps from $X$ to $Y$. Let also $\Sigma$ be the set of $(x, y) \in X \times Y$ for which there exists a sequence $\left\{x_{n}\right\}_{n \in \mathbb{Z}_{+}}$of elements of $X$ such that $x_{n} \rightarrow x$ and $T_{n} x_{n} \rightarrow y$ as $n \rightarrow \infty$. If $\sum$ is dense in $X \times Y$, then $\left\{T_{n}\right\}_{n \in \mathbb{Z}_{+}}$is hereditarily universal.
Proof. The density of $\Sigma$ in $X \times Y$ implies that for any infinite set $A \subseteq \mathbb{Z}_{+}$, condition (U3) from Theorem $U$ is satisfied for the family $\left\{T_{n}: n \in A\right\}$ and therefore this family is universal.

Hilden and Wallen [30] demonstrated that any backward weighted shift on $\ell_{\mathrm{p}}$ for $1 \leq p<\infty$ is supercyclic. Many particular cases of the following proposition are known, see for instance [31]. We include it here in its full generality for the sake of completeness.

Proposition 5.7 Let $X$ be a Baire separable metrizable topological vector space $T \in L(X)$. Suppose also that $T$ has dense range and dense generalized kernel. Then $T$ is hereditarily supercyclic.

Proof. Since $T$ has dense range, we have that $T^{k}(X)$ is dense in $X$ for each $k \in \mathbb{Z}_{+}$. Thus for any $x \in X$ there exists a sequence $\left\{u_{k}^{x}\right\}_{k \in \mathbb{Z}_{+}}$in $X$ such that $T^{k} u_{k}^{x} \rightarrow x$ as $k \rightarrow \infty$. Fix a dense countable set $B \subseteq X$. Since $X$ is metrizable and $B$ is countable, we can choose a sequence $\left\{\lambda_{k}\right\}_{k \in \mathbb{Z}_{+}}$ of positive numbers such that $\lambda_{k}^{-1} u_{k}^{x} \rightarrow 0$ as $k \rightarrow \infty$ for each $x \in B$.

Let now $T_{k}=\lambda_{k} T^{k}$ for each $k \in \mathbb{Z}_{+}$and $\Sigma$ be the set of $(x, y) \in X \times X$ for which there exists a sequence $\left\{x_{k}\right\}_{k \in \mathbb{Z}_{+}}$of elements of $X$ such that $x_{k} \rightarrow x$ and $T_{k} x_{k} \rightarrow y$ as $k \rightarrow \infty$. For any $x \in B$, let $x_{k}=\lambda_{k}^{-1} u_{k}^{x}$. Then $x_{k} \rightarrow 0$ as $k \rightarrow \infty$ and $T_{k} x_{k}=x$ for each $k \in \mathbb{Z}_{+}$. Hence $\{0\} \times B \subseteq \Sigma$. On the other hand, for any $x \in k e r{ }^{\star} T$, we have $T_{k} x=0$ for all sufficiently large $k$ and therefore $T_{k} x \rightarrow 0$ as $k \rightarrow \infty$. Considering the constant sequence $x_{k}=x$ for $k \in \mathbb{Z}_{+}$, we see that $\operatorname{ker}^{*} T \times\{0\} \subseteq \Sigma$.

Finally, observe that $\Sigma$ is a linear subspace of $X \times X$. Hence $k e r{ }^{\star} T \times B \subseteq \Sigma$. Since both $B$ and $k e r{ }^{*} T$ are dense in $X$, we see that $\Sigma$ is dense in $X \times X$. By Lemma 5.6, for each infinite set $A \subseteq \mathbb{Z}_{+}$, the family $\left\{T_{n}: n \in A\right\}=\left\{\lambda_{n} T^{n}: n \in A\right\}$ is universal. Hence $T$ is hereditarily supercyclic.

\section{Remarks on Theorem 1.18}

Theorem 1.18 is reminiscent of the following criterion of hypercyclicity of Bayart and Grivaux [32] in terms of the unimodular point spectrum.

Theorem BG Let $X$ be a complex separable infinite dimensional Banach space, $T \in L(X)$ and assume that there exists a continuous Borel probability measure $\mu$ on the unit circle $\mathbb{T}$ such that for each Borel set $A \subseteq \mathbb{T}$ with $\mu(A)=1$, the space

$$
\operatorname{span}\left(\bigcup_{z \in A} \operatorname{ker}(T-z I)\right)
$$

is dense in $X$. Then $T$ is hypercyclic.

It is worth noting that in the case of operators on Banach spaces, neither Theorem 1.18 implies the result of Bayart and Grivaux, nor their result implies Theorem 1.18, see Examples 5.9, 5.10 and 5.12 below. Theorem 1.18 is also strictly stronger than Proposition 2.2 in the article [33] by Herrero and Wang, which is a key tool in the proof of the main result in reference [33] that any element of the operator norm closure of the set of hypercyclic operators on $\ell_{2}$ is a compact perturbation of a hypercyclic operator. Namely, they assume that the span taken in (1) is dense taking only into account the $z$ 's for which $T-z I$ has closed range, and this allows them to use the Kitai criterion. We would like also to mention the following fact.

Proposition 5.8 Let $X$ be a locally convex space and $Y$ be a closed linear subspace of $X$ such that $X$ admits an $\ell_{1}$-sequence with dense 
span and the topology of $X / Y$ is not weak. Then there is a hereditarily hypercyclic operator $T \in L(X)$ such that $T y=y$ for any $y \in Y$.

Proof. Since the topology of $X / Y$ is not weak, Lemma 3.1 implies that there is a linearly independent uniformly equicontinuous sequence $\left\{g_{n}\right\}_{n \in \mathbb{Z}_{+}}$such that $Y \subseteq k e r g_{n}$ for each $n \in \mathbb{Z}_{+}$. By Lemma 3.5, we can find sequences $\left\{x_{n}\right\}_{n \in \mathbb{Z}_{+}}$and $\left\{f_{k}\right\}_{k \in \mathbb{Z}_{+}}$in $X$ and $X^{\prime}$ respectively, such that conditions (3.5.1-3.5.4) are satisfied and each $f_{k}$ belongs to $\operatorname{span}\left\{g_{m}: m \in \mathbb{Z}_{+}\right\}$. Hence $Y \subseteq k e r f_{k}$ for any $k \in \mathbb{Z}_{+}$. Uniform equicontinuity of $\left\{f_{k}\right\}$ and the fact that $\left\{x_{k}\right\}$ is an $\ell_{1}$-sequence implies that the formula

$$
T x=\sum_{n=0}^{\infty} 2^{-n} f_{n+1}(x) x_{n}
$$

defines a continuous linear operator on $X$, which also acts continuously on the Banach space $X_{K}$, where $K$ is the Banach disk being the closed convex balanced hull of $\left\{x_{k}: k \in \mathbb{Z}_{+}\right\}$. By Lemma 3.3, $X_{K}$ is separable and $E=\operatorname{span}\left\{x_{k}: k \in \mathbb{Z}_{+}\right\}$is dense in $X_{K}$. It is also straightforward to verify that $\operatorname{ker}^{\dagger}\left(T_{K}\right)$ contains $E$, where $T_{k} \in L\left(X_{k}\right)$ is the restriction of $T$ to $X_{K}$. Hence $T_{K}$ is an extended backward shift on the separable Banach space $X_{K}$. By Corollary 5.1, $I+T_{k}$ is hereditarily hypercyclic. Since $X_{K}$ is dense in $X$ and carries stronger topology, $I+T \in L(X)$ is hereditarily hypercyclic. Since $Y \subseteq k e r f_{k}$ for each $k \in \mathbb{Z}_{+}$from the definition of $T$ it follows that $(I+T) y=y$ for each $y \in Y$. Thus $I+T$ satisfies all required conditions.

Grivaux [34] proved that if $Y$ is a closed linear subspace of a separable Banach space $X$ such that $X / Y$ is infinite dimensional, then there exists a hypercyclic $T \in L(X)$ such that $T y=y$ for any $y \in Y$. This result is an immediate corollary of Proposition 5.8. If we apply Proposition 5.8 in the case when $X$ is a separable Fréchet space, we obtain that whenever $Y$ is a closed linear subspace of $X$ such that $X / Y$ is infinite dimensional and non-isomorphic to $\omega$, there is a hereditarily hypercyclic $T \in L(X)$ such that $T y=y$ for any $y \in Y$. It is also worth noting that any non-normable Fréchet space has a quotient isomorphic to $\omega$, [29]. If $X$ is a non-normable separable Fréchet space non-isomorphic to $\omega$ and $Y$ is a closed linear subspace of $X$ such that $X / Y$ is isomorphic to $\omega$, then there is no hypercyclic operator $T \in L(X)$ such that $T y=y$ for each $y \in Y$. Indeed, assume that such a $T$ does exist. Then $S: X / Y \rightarrow X$ ,$S(x+Y)=T x-x$ is a continuous linear operator with dense range from $X / Y$ to $X$. Since $X / Y$ is isomorphic to $\omega$ and $\omega$ carries the minimal locally convex topology, $S$ is onto and therefore $X$ is isomorphic to $\omega$, which is a contradiction.

Example 5.9 Let $\mathcal{H}$ be the complex Sobolev space $W^{-1,2}(\mathbb{T})$ which consists of the distributions $f$ on $\mathbb{T}$ such that $\sum_{n=-\infty}^{\infty}\left|\widehat{f}_{n}\right|^{2}\left(1+n^{2}\right)^{-1}<\infty$, where the $\widehat{f}_{n}$ denotes the $n^{\text {th }}$ Fourier coefficient of $f$. Then the operator $T$ acting on $\mathcal{H}$ as $\operatorname{Tf}(z)=z f(z)$ satisfies the conditions of Theorem BG (and therefore is hypercyclic) and does not satisfy the conditions of Theorem 1.18 .

Proof. For each $z \in \mathbb{T}$, $\operatorname{ker}(T-z I)=k e r^{*}(T-z I)$ is the onedimensional space spanned by the Dirac $\delta$-function $\delta$, which does not belong to the range of $T-z I$. Thus $\Lambda(T)=\{0\}$ and $T$ does not satisfy conditions of Theorem 1.18. On the other hand, $\operatorname{span}\left\{\delta_{z}: z \in A\right\}$ is dense in $\mathcal{H}$ for any set $A \subseteq \mathbb{T}$ which is dense in $\mathbb{T}$. Since any subset of $\mathbb{T}$ of full Lebesgue measure is dense, the conditions Theorem BG are satisfied with $\mu$ being the normalized Lebesgue measure.
Example 5.10 If $\mathcal{H}$ is the complex Sobolev space $W^{-2,2}(\mathbb{T})$ which consists of the distributions $\mathrm{f}$ on $\mathbb{T}$ such that $\sum_{n=-\infty}^{\infty}\left|\hat{f}_{n}\right|^{2}\left(1+n^{2}\right)^{-2}<\infty$, then the operator $\operatorname{Tf}(z)=z f(z)$ acting on $\mathcal{H}$ satisfies the conditions of both Theorem BG and Theorem 1.18.

Proof. For each $z \in \mathbb{T}, \delta_{z} \in \operatorname{ker}(T-z I) \cap(T-z I)(\mathcal{H})$ and therefore the dense linear span of the set $\left\{\delta_{z}: z \in \mathbb{T}\right\}$ is contained in $\Lambda(T)$. Hence $T$ satisfies the conditions of Theorem 1.18. As in the above example, $\operatorname{span}\left\{\delta_{z}: z \in A\right\}$ is dense in $\mathcal{H}$ for any set $A \subseteq \mathbb{T}$ which is dense in $\mathbb{T}$ and therefore $T$ satisfies conditions of Theorem BG.

Example 5.11 If $T$ is a quasinilpotent generalized backward shift on a separable complex Banach space, then $I+T$ satisfies the conditions of Theorem 1.18 and does not satisfy the conditions of Theorem BG.

Proof. Since $\sigma_{p}(I+T)=\{1\}$, conditions of Theorem BG are not satisfied for the operator $I+T$. On the other hand, $\operatorname{ker}^{\dagger} T \subseteq \Lambda(I+T)$ is dense and therefore $I+T$ satisfies conditions of Theorem 1.18.

Examples of chaotic operators on a complex Hilbert space $H$ which are not mixing are constructed in [35]. For such an operator, the linear span of the union of $\operatorname{ker}(T-z I)$ for $z \in \mathbb{T}$ satisfying $z^{n}=1$ for some $n \in \mathbb{N}$ is dense in $H$. This shows that the assumption of Theorem 1.18 cannot be relaxed into a weaker assumption like density of the linear span of the union of $k e r(T-z I)^{n}$ for $z \in \mathbb{T}$ and $n \in \mathbb{Z}$. Note also that the class of operators $T$ for which $\Lambda(T)$ is dense is closed under finite direct sums. Moreover, this class for operators acting on Banach spaces is closed under infinite $c_{0}$-sums and $\ell_{\mathrm{p}}$-sums for $1 \leq p<\infty$. In particular, $I+T$ is mixing, when $T$ is a finite or countable $c_{0 .}$ sum or $\ell_{\mathrm{p}}$ sum with $1 \leq p<\infty$ of (possibly different) backward weighted shifts.

Now we describe another class of operators to which Theorem 1.18 applies. Let $\alpha \in L_{\infty}[0,1]$ and $\varphi:[0,1] \rightarrow[0,1]$ be a Borel measurable map. Consider the integral operator $T_{\alpha \varphi}$ defined by

$$
T_{\alpha, \varphi} f(x)=\int_{0}^{\varphi(x)} \alpha(t) f(t) d t .
$$

It is straightforward to verify that $T_{\alpha \varphi}$ is a compact linear operator on $L_{p}[0,1]$ for $1 \leq p \leq \infty$.

Example 5.12 Assume that $\Psi$ is continuous, strictly increasing, $\psi(x)<x$ for $0<x \leq 1$ and that $\alpha(x) \neq 0$ almost everywhere on [0.1]. Then $T=T_{\alpha, \psi}$ acting on $L_{p}[0,1]$ for $1 \leq p<\infty$ is hereditarily supercyclic and $\mathrm{I}+\mathrm{T}$ is mixing.

Proof. Consider the sequence $a_{1}=\psi(1)$ and $a_{n+1}=\psi\left(a_{n}\right)$ for $n \in$ $\mathbb{N}$. Clearly $\left\{a_{n}\right\}$ is strictly decreasing and tends to zero as $n \rightarrow \infty$. One can easily verify that

$$
\operatorname{ker} T^{n}=\left\{f:\left.f\right|_{\left[0, a_{n}\right]}=0\right\} \text {. }
$$

Using this equality it is straightforward to check that $T\left(\operatorname{ker} T^{n+1}\right)$ is dense in $\operatorname{kerT}^{n}$ for each $n \in \mathbb{N}$ and that $\operatorname{ker}^{\star} T$ is dense in the entire space. Thus $k e r^{\dagger} T$ is dense in $L_{p}[0,1]$. That is, $T$ is an extended backward shift. It remains to apply Proposition 5.7 and Corollary 5.1.

\section{Universality of generic families}

Theorem 6.1 Let $X$ be a separable metrizable Baire topological space, $\Omega$ be a Baire topological space, $A$ be a set, and for each $a \in A$ let $\Psi$ be a map from $\Omega$ into the set $C(X, X)$ of continuous maps from $X$ to $X$ such that 
for any $\mathrm{a} \in \mathrm{A}$,themap $\Phi_{\mathrm{a}}: \Omega \times \mathrm{X} \rightarrow \mathrm{X}, \Phi_{\mathrm{a}}(\alpha, \mathrm{X})=\Psi_{\mathrm{a}}(\alpha) \mathrm{X}$ is continuous (6.1)

and $\left\{\left(\alpha, \mathrm{x}, \Psi_{\mathrm{a}}(\alpha) \mathrm{x}\right): \alpha \in \Omega, \mathrm{x} \in \mathrm{X}, \mathrm{a} \in \mathrm{A}\right\}$ is dense in $\Omega \times \mathrm{X} \times \mathrm{X}$.

Then

$U=\left\{\alpha \in \Omega:\left\{\Psi_{\mathrm{a}}(\alpha): \mathrm{a} \in \mathrm{A}\right\}\right.$ is universal $\}$ is a dense $\mathrm{G}_{\delta}$ - subset of $\Omega$.

Proof. Let $\left\{{ }_{n}\right\}_{n \in}$ be a sequence of non-empty open subsets of $X$, which form a basis of the topology of $X$. By Theorem $U$, $\left\{\Psi_{a}(\alpha): a \in A\right\}$ is universal if and only if for each $k, m \in \mathbb{Z}_{+}$there exists $a \in A$ for which $\Phi_{a}(\alpha)\left(U_{k}\right) \cap U_{m} \neq \varnothing$. Thus we have

$$
U=\bigcap_{k, m=0}^{\infty} \bigcup_{x \in U_{k}} \bigcup_{a \in A}\left\{\alpha \in \Omega: \Phi_{a}(\alpha, x) \in U_{m}\right\}
$$

According to (18), the sets $\left\{\alpha \in \Omega: \Phi_{a}(\alpha, x) \in U_{m}\right\}$ are open in $\Omega$. Hence, the above display implies that $U$ is a $G_{\delta}$-subset of $\Omega$. It remains to show that $U$ is dense in $\Omega$. Let

$$
U_{0}=\left\{(\alpha, x) \in \Omega \times X: \mathrm{x} \text { is a universal element for }\left\{\Psi_{\mathrm{a}}(\alpha): \mathrm{a} \in \mathrm{A}\right\}\right\} .
$$

Clearly $U$ is the projection of $U_{0}$ onto $\Omega$. On the other hand, $U_{0}$ is the set of universal elements of the family $\left\{\Phi_{a}: a \in A\right\}$. Since the product of two Baire spaces, one of which is second countable, is Baire [36], $\Omega \times X$ is Baire. Applying Theorem $U$, we see that $U_{0}$ is dense in $\Omega$ $\times X$. Since the projection onto $\Omega$ of a dense subset of $\Omega \times X$ is dense, we get that $U$ is dense in $\Omega$.

We apply the above general result to two types of universality: hypercyclicity and supercyclicity.

Theorem 6.2 Let $X$ be a separable metrizable Baire topological vector space, $\Omega$ be a Baire topological space and $\alpha \mapsto T_{\alpha}$ be a map from $\Omega$ intoL $(X)$ such that

for any $\mathrm{n} \in \mathbb{Z}_{+}$, the map $\Phi_{\mathrm{n}}: \Omega \times \mathrm{X} \rightarrow \mathrm{X}, \Phi_{\mathrm{n}}(\alpha, \mathrm{x})=\mathrm{T}_{\alpha}^{\mathrm{n}} \mathrm{x}$ is continuous.

Then the following conditions are equivalent :

$\left\{\left(\alpha, \mathrm{x}, \mathrm{T}_{\alpha}^{\mathrm{n}} \mathrm{x}\right): \alpha \in \Omega, \mathrm{x} \in \mathrm{X}, \mathrm{n} \in \mathbb{Z}_{+}\right\}$is dense in $\Omega \times \mathrm{X} \times \mathrm{X}$,

$H=\left\{\alpha \in \Omega: \mathrm{T}_{\alpha}\right.$ is hypercyclic $\}$ is a dense $\mathrm{G}_{\delta}$ - subset of $\Omega$.

Proof. Let $A \in \mathbb{Z}_{+}$and $\Psi_{n}: \Omega \rightarrow L(X)$ for $n \in A$ be defined as $\Psi_{n}(\alpha)=T_{\alpha}^{n}$. Applying Theorem 6.1, we see that (6.4) implies (6.5). Since the set of hypercyclic vectors of any hypercyclic operator is dense, (6.5) implies (6.4).

Theorem 6.3 Let $X$ be a separable metrizable Baire topological vector space, $\Omega$ be a Baire topological space and $\alpha \mapsto T_{\alpha}$ be a map from $\Omega$ to $L(X)$ such that (6.3) is satisfied. Then the following conditions are equivalent:

$\left\{\left(\alpha, \mathrm{x}, \lambda \mathrm{T}_{\alpha}^{\mathrm{n}} \mathrm{x}\right): \alpha \in \Omega, \mathrm{x} \in \mathrm{X}, \mathrm{n} \in \mathbb{Z}_{+}, \lambda \in \mathbb{C}\right\}$ is dense in $\Omega \times \mathrm{X} \times \mathrm{X}$,

$S=\left\{\alpha \in \Omega: \mathrm{T}_{\alpha}\right.$ is supercyclic $\}$ is a dense $\mathrm{G}_{\delta}-$ subset of $\Omega$.

Proof. Let $A=\mathbb{Z}_{+} \times \mathbb{C}$ and $\Psi_{n, \lambda}: \Omega \rightarrow L(X)$ for $(n, \lambda) \in A$ be defined as $\Psi_{n, \lambda}(\alpha)=\lambda T_{\alpha}^{n}$. Applying Theorem 6.1, we see that (6.6) implies (6.7). Since the set of supercyclic vectors of any supercyclic operator is dense, (6.7) implies (6.6).

Corollary 6.4 Let $X$ be a separable Baire metrizable topological vector space, $\Omega$ be a Baire topological space and $\alpha \mapsto T_{\alpha}$ be a map from $\Omega$ to $L(X)$ satisfying (6.3). Suppose also that for any non-empty open subset $W$ of $\Omega$ and any nonempty open subsets $U, V$ of $X$, there exist $\alpha \in W, x \in U$ and $y \in V$ such that $x, y \in \Lambda\left(T_{\alpha}\right)$, where $\Lambda\left(T_{\alpha}\right)$ is defined in (1.1). Then the set of $\alpha \in \Omega$ for which $T_{\alpha}$ is hypercyclic is a dense $G$-subset of $\Omega$.

Proof.Let $(\alpha, x, y) \in \Omega \times X \times X$ besuchthat $x, y \in \Lambda\left(T_{\alpha}\right)$. ByTheorem $2.12(x, y)$ is in the closure of the set $\left\{\left(u, T_{\alpha}^{n} u\right): u \in X, n \in \mathbb{Z}_{+}\right\}$. Hence $(\alpha, x, y)$ is in the closure of the set $\left\{\left(\alpha, u, T_{\alpha}^{n} u\right): \alpha \in \Omega, u \in X, n \in \mathbb{Z}_{+}\right\}$. By the assumptions of the corollary, the last set is dense in $\Omega \times X \times X$. It remains to apply Theorem 6.2.

The following supercyclicity analog of the above corollary turns out to be much easier.

Proposition 6.5 Let $X$ be a Baire separable metrizable topological vector space, $\Omega$ be a Baire topological space and $\alpha \mapsto T_{\alpha}$ be a map from $\Omega$ to $L(X)$ satisfying (6.3). Suppose also that for any non-empty open subset $W$ of $\Omega$ and any nonempty open subsets $U$ and $V$ of $X$, there exist $\alpha \in W, x \in U, y \in V$ and $n \in \mathbb{N}$ such that $x \in \operatorname{ker} T_{\alpha}^{n}$ and $y \in T_{\alpha}^{n}(X)$. Then the set of $\alpha \in \Omega$ for which $T_{\alpha}$ is supercyclic is a dense $G_{\delta}$-subset of $\Omega$.

Proof. Let $(\alpha, x, y) \in \Omega \times X \times X$ be such that there exists $n \in \mathbb{N}$ for which $x \in \operatorname{ker} T_{\alpha}^{n}$ and $y \in T_{\alpha}^{n}(X)$. Let $v \in X$ be such that $T_{\alpha}^{n} v=y$. For each $m \in \mathbb{N}$ consider $u_{m}=x+m^{-1} v$. Then $u_{m} \rightarrow x$ as $m \rightarrow \infty$. Moreover, $m T^{n} u_{m}=y$ for any $m \in \mathbb{N}$. Thus for all sufficiently large $m$, $\left(\alpha, u_{m}, m T^{n} u_{m}\right) \in W \times U \times V$. Since $W, U$ and $V$ were arbitrary, the set $\left\{\left(\alpha, u, z T^{n} u\right): \alpha \in \Omega, n \in \mathbb{Z}_{+}, z \in \mathbb{K}\right\}$ is dense in $\Omega \times X \times X$. It remains to apply Theorem 6.3 .

The obvious inclusion $\operatorname{ker}^{\dagger}(T-I) \subset \Lambda(T)$ and the fact that a map $\alpha \rightarrow T_{\alpha}$ satisfies (6.3) if and only if the map $\alpha \rightarrow I+T_{\alpha}$ satisfies (6.3) imply the following corollary of Corollary 6.4 and Proposition 6.5.

Corollary 6.6 Let $X$ be a Baire separable metrizable topological vector space, $\Omega$ be a Baire topological space and $\alpha \mapsto T_{\alpha}$ be a map from $\Omega$ to $L(X)$ satisfying (6.3). Suppose also that for any non-empty open subset $W$ of $\Omega$ and any nonempty open subsets $U$ and $V$ of $X$, there exist $\alpha \in W, x \in U$ and $y \in V$ such that $x, y \in$ ker $^{\dagger} T_{\alpha}$. Then the set of $\alpha \in \Omega$ for which $T_{\alpha}$ is supercyclic and $I+T_{\alpha}$ is hypercyclic is a dense $G_{\delta}$-subset of $\Omega$.

Now we apply the above general results. In particular, we shall prove Theorems 1.19 and 1.21 . For a while we shall assume that $X$ and $Y$ are two infinite dimensional Banach spaces, $b: X \times Y \rightarrow \mathbb{K}$ is a continuous bilinear form separating points of $X$ and of $Y$. That is, for each non-zero $x \in X$, there is $y \in Y$ satisfying $b(x, y) \neq 0$ and for each non-zero $y \in Y$, there is $x \in X$ such that $b(x, y) \neq 0$. In particular, $b$ is a dual pairing between $X$ and $Y$. Recall that the injective norm on the tensor product $X \otimes Y$ is defined by the formula

$$
\varepsilon(\xi)=\sup \left\{\left|\sum_{j=1}^{n} \phi\left(x_{j}\right) \psi\left(y_{j}\right)\right|: \phi \in X^{\prime}, \psi \in Y^{\prime},\|\phi\| \leq 1,\|\psi\| \leq 1\right\} \text { for } \xi=\sum_{j=1}^{n} x_{j} \otimes y_{j} \in X \otimes Y .
$$

The completion of $X \otimes Y$ with respect to this norm is called the injective tensor product of $X$ and is $Y$ and denoted $X \widehat{\otimes}_{\varepsilon} Y$. It is again a Banach space. For each $\xi \in X \otimes Y$ we consider the linear operators $T_{\xi} \in L(X)$ and $S_{\xi} \in L(Y)$ defined by the formulae

$$
T_{\xi} x=\sum_{j=1}^{n} b\left(x, y_{j}\right) x_{j} \text { and } S_{\xi} y=\sum_{j=1}^{n} b\left(x_{j}, y\right) y_{j} \text {, where } \xi=\sum_{j=1}^{n} x_{j} \otimes y_{j} .
$$

Clearly $\xi \mapsto T_{\xi}$ and $\xi \mapsto S_{\xi}$ are bounded linear operators from $X \otimes Y$ endowed with the injective norm into the Banach spaces $L(X)$ 
and $L(Y)$ respectively. Hence they admit unique continuous extensions to $X \widehat{\otimes}_{\mathcal{E}} Y$, which we again denote by $\xi \mapsto T_{\xi}$ and $\quad$. Note that $S_{\xi}$ is the dual operator of $T_{\xi}$ with respect to the dual pairing $b$ :

$$
b\left(T_{\xi} x, y\right)=b\left(x, S_{\xi} y\right) \text { foranyx } \in \mathrm{Xand} \mathrm{y} \in \mathrm{Y} .
$$

Let now $\mathcal{M}$ be a linear subspace of $X \widehat{\otimes}_{\varepsilon} Y$ which we suppose to be endowed with its own $\mathcal{F}$-space topology stronger than the one inherited from $X \widehat{\otimes}_{\varepsilon} Y$. Suppose also that $X \otimes Y$ is a dense linear subspace of $\mathcal{M}$ and let $\mathcal{M}^{\mathcal{N}}$ be the closure in $\mathcal{M}$ of the set $\left\{\xi \in X \otimes Y: T_{\xi}\right.$ is nilpotent $\}$. Remark that (6.9) implies that $T_{\xi}^{n}=0$ if and only if $S_{\xi}^{n}=0$. Thus nilpotency of $T_{\xi}$ is equivalent to nilpotency of $S_{\xi}$ and this implies that $\mathcal{M}^{\mathcal{N}}$ coincides with the closure in $\mathcal{M}$ of the set $\left\{\xi \in X \otimes Y: S_{\xi}\right.$ isnilpotent $\}$.

\section{Proposition 6.7 If the Banach space $X$ is separable, then the set}

$$
\left\{\xi \in \mathcal{M}^{\mathcal{N}}: T_{\xi} \text { is supercyclic and } I+T_{\xi} \text { is hypercyclic }\right\}
$$

is a dense $G_{\delta}$ subset of $\mathcal{M}^{\mathcal{N}}$. If $X$ and $Y$ are both separable, then the set

$$
\left\{\xi \in \mathcal{M}^{\mathcal{N}}: \mathrm{T}_{\xi} \text { and } \mathrm{S}_{\xi} \text { are supercyclic and } \mathrm{I}+\mathrm{T}_{\xi} \text { and } \mathrm{I}+\mathrm{S}_{\xi} \text { are hypercyclic }\right\}
$$

is a dense $G_{\delta}$ subset of $\mathcal{M}^{\mathcal{N}}$.

Proof. Let $W$ be a non-empty open subset of $\mathcal{M}^{\mathcal{N}}$ and $U_{1}, U_{2}$ be nonempty open subsets of $X$. Pick $x_{1} \in U_{1}$ and $x_{2} \in U_{2}$. By the definition of $\mathcal{M}^{\mathcal{N}}$, there exists $\xi \in X \otimes Y$ such that $\xi \in W$ and $T_{\xi}$ is nilpotent. Let $n$ $\in \mathbb{N}$ be such that $T_{\xi}^{n}=0$. Clearly the space

$$
L=\left\{y \in Y: S_{\xi} y=0, b\left(x_{1}, y\right)=b\left(x_{2}, y\right)=0\right\}
$$

has finite codimension in $Y$, while $\operatorname{ker} T_{\xi}$ has finite codimension in $X$. Since $b$ is a dual pairing of $X$ and $Y$, we can find $f \in L$ and $u_{j} \in \operatorname{ker} T_{\xi}$ for $1 \leq j \leq 2 n$ such that $b\left(u_{k}, f_{j}\right)=\delta_{k, j}$ for $1 \leq j, k \leq 2 n$. Consider the element

$$
\eta=x_{1} \otimes f_{1}+x_{2} \otimes f_{n+1}+\sum_{j=2}^{n}\left(u_{j-1} \otimes f_{j}+u_{n+j-1} \otimes f_{n+j}\right) \in X \otimes Y
$$

and let $\xi_{t}=\xi+t \eta$ for $t \in \mathbb{K}$. From the above properties of $f_{j}$ and $u_{j}$ it immediately follows that the range of $T_{\xi_{t}}^{n}$ is contained in the range $Q$ of $T_{\xi}$ and that the restrictions of $T_{\xi}$ and $T_{\xi}$ to $Q$ coincide. Hence $T_{\xi_{t}}^{2 n}=0$ for any $t \in \mathbb{K}$. Thus all $T_{\xi_{t}}$ are nilpotent and therefore all $\xi_{t}$ belong to $\mathcal{M}^{\mathcal{N}}$. Since $W$ is open in $\mathcal{M}^{\mathcal{N}}$ we can pick $s \in \mathbb{K} \backslash\{0\}$ close enough to zero to ensure that $\xi_{s} \in W$. Now from the definition of $\xi_{s}$ it immediately follows that $T_{\xi_{s}}^{n} u_{n}=s^{n} x_{1}, T_{\xi_{s}}^{n} u_{2 n}=s^{n} x_{2}, T_{\xi_{s}}^{n} x_{1}=T_{\xi}^{n} x_{1}=0$ and $T_{\xi_{s}}^{n} x_{2}=T_{\xi}^{n} x_{2}=0$. Thus

$$
x_{1}, x_{2} \in \operatorname{ker} T_{\xi_{s}}^{n} \cap T_{\xi_{s}}^{n}(X) \subset \operatorname{ker}^{\dagger} T_{\xi_{s}} .
$$

Summarizing the above, we have found $x_{1} \in U_{1}, x_{2} \in U_{2}$ and $\xi_{s} \in W$ such that $x_{1}, x_{2} \in \operatorname{ker}^{\dagger} T_{\xi_{s}}$. Using the fact that $\mathcal{M}^{\mathcal{N}}$ carries a topology stronger than the one defined by the injective norm, we see that the map $\xi \mapsto T_{\xi}$ from $\mathcal{M}^{\mathcal{N}}$ to $L(X)$ satisfies (6.3). By Corollary 6.6, the set of $\xi \in \mathcal{M}^{\mathcal{N}}$ for which $T_{\xi}$ is supercyclic and $I+T_{\xi}$ is hypercyclic is a dense $G_{\delta}$ subset of $\mathcal{M}^{\mathcal{N}}$. The proof of the first part of the proposition is complete.

Applying the first part of the proposition to $(Y, X)$ instead of $(X, Y)$ with $b(x, y)$ replaced by $b(y, x)$, we obtain that if $Y$ is separable, then the set of $\xi \in \mathcal{M}^{\mathcal{N}}$ for which $S_{\xi}$ is supercyclic and $I+S_{\xi}$ is hypercyclic is a dense $G_{\delta}$ subset of $\mathcal{M}^{\mathcal{N}}$. The second part now follows from the first part and the fact that the intersection of two dense $G_{\delta}$ subsets of a complete metric space is again a dense $G_{\delta}$ set.

\section{Proof of Theorems 1.19 and 1.21}

Let us consider the case where $Y=X^{\prime}, b(x, y)=y(x)$ and $\mathcal{M}=X \widehat{\otimes}_{\varepsilon} X^{\prime}$. In this case the map $\xi \mapsto T_{\xi}$ is an isometry and $\left\{T_{\xi}: \xi \in \mathcal{M}^{\mathcal{N}}\right\}$ is exactly the operator norm closure of the set of finite rank nilpotent operators. Moreover, taking into account that $T_{\xi}^{\prime}=S_{\xi}$ for any $\xi \in \mathcal{M}$, we see that Theorem 1.19 is an immediate corollary of Proposition 6.7 for this specific choice of $M$ and $b$.

Recall that the projective norm on the tensor product $X \otimes Y$ of the Banach spaces $X$ and $Y$ is defined by the formula

$$
\pi(\xi)=\inf \left\{\sum\left\|x_{j}\right\|\left\|y_{j}\right\|\right\},
$$

where the infimum is taken over all possible representations of $\xi$ as a finite sum $\sum x_{j} \otimes y_{j}$. If we consider the case $Y=X^{\prime}, b(x, y)=y(x)$ and $\mathcal{M}=X \widehat{\otimes}_{\pi} X^{\prime}$ the completion of $X \otimes X^{\prime}$ with respect to the projective norm, then $\left\{T_{\xi}: \xi \in \mathcal{N}\right\}$ is exactly the set of nuclear quasinilpotent operators, and we see that Theorem 1.21 is an immediate corollary of Proposition 6.7 for this choice of $M$ and $b$.

\section{Proof of Theorem 1.6}

Let $X \in \mathfrak{M}$ By Lemma 3.5, there exist sequences $\left\{x_{n}\right\}_{n \in \mathbb{Z}_{+}}$and $\left\{f_{n}\right\}_{n \in \mathbb{Z}_{+}}$in $X$ and $X^{\prime}$ respectively such that conditions (3.5.1-3.5.4) are satisfied. Since $\left\{f_{n}\right\}$ is uniformly equicontinuous, we can pick a nonzero continuous seminorm $p$ on $X$ such that $\left|f_{n}(x)\right| \leq 1$ for any $n \in \mathbb{Z}_{+}$ whenever $p(x) \leq 1$. By Lemma 3.3, the closed balanced convex hull $K$ of $\left\{x_{n}: n \in \mathbb{Z}_{+}\right\}$a Banach $s$-disk. That is, the Banach space $X_{k}$ is separable. It is also clear from Lemma 3.3 that any $x \in X_{K}$ has shape $x=\sum_{n=0}^{\infty} a_{n} x_{n}$ for some $a \in \ell_{1}$ and $\|x\| \leq\|a\|_{1}$, where $\|x\|$ is the norm of $X$ in the Banach space $X_{k . .}$ Consider the bilinear form on $X \times \ell_{1}$ defined by the formula

$$
b(x, a)=\sum_{n=0}^{\infty} a_{n} f_{n}(x) \text {. }
$$

It is easy to see that $b$ is well-defined, continuous and $b(x, a) \leq p(x)\|a\|_{1}$ for any $x \in X$ and $a \in \ell_{1}$. Moreover, $b$ separates points of $X_{k}$ and $\ell_{1}$. Indeed, let $x \in X_{K}, x \neq 0$. Pick $\alpha \in \ell_{1}$ such that $x=\sum_{n=0}^{\infty} \alpha_{n} x_{n}$. Since $x \neq 0$, there is $m \in \mathbb{Z}_{+}$such that $\alpha_{m} \neq 0$. Using (3.5.3), we see that $b\left(x, e_{m}\right)=f_{m}(x)=\alpha_{m} f_{m}\left(x_{m}\right) \neq 0$, where $\left\{e_{j}\right\}_{j \in \mathbb{Z}_{+}}$is the standard basis in $\ell_{1}$. Similarly, let $a \in \ell_{1}, a \neq 0$. Then there is $m \in \mathbb{Z}_{+}$for which $a_{m} \neq 0$ and therefore, according to (3.5.3), $b\left(x_{m}, a\right)=a_{m} f_{m}\left(x_{m}\right) \neq 0$. Thus $b$ separates points of $X_{k}$ and $\ell_{1}$. Let $\mathcal{M}=X_{K} \widehat{\otimes}_{\pi} \ell_{1}$ be the projective tensor product of the Banach spaces $X_{k}$ and $\ell_{1}$ and $\mathcal{M}^{\mathcal{N}}$ be the closure in $\mathcal{M}$ of the set of $\xi \in X_{K} \otimes \ell_{1}$, for which the operator $T_{\xi}$ defined in (6.8) is nilpotent. By Proposition 6.7, the set $\left\{\xi \in \mathcal{M}^{\mathcal{N}}: I+T_{\xi}\right.$ and $I+S_{\xi}$ are both hypercyclic $\}$ is a dense $G_{\delta}$ subset of $\mathcal{M}^{\mathcal{N}}$. In particular, we can pick $\xi \in \mathcal{M}^{\mathcal{N}} \subset X_{D} \widehat{\otimes}_{\pi} \ell_{1}$ such that $I+T_{\xi}$ and $I+S_{\xi}$ arehypercyclic. Using the theorem characterizing the shape of elements of the projective tensor product [17], we see that there exist bounded sequences $\left\{y_{n}\right\}_{n \in \mathbb{Z}_{+}}$and $\left\{w_{n}\right\}_{n \in \mathbb{Z}_{+}}$in $X_{k}$ 
and $\ell_{1}$ respectively and $\lambda \in \ell_{1}$ such that $\xi=\sum_{n=0}^{\infty} \lambda_{n} y_{n} \otimes w_{n}$. Then the operators $T_{\xi}$ and $S_{\xi}$ act according to the following formulae on $X_{k}$ and $\ell_{1}$ respectively:

$$
T_{\xi} x=\sum_{n=0}^{\infty} \lambda_{n} b\left(x, w_{n}\right) y_{n} \text {, and } S_{\xi} a=\sum_{n=0}^{\infty} \lambda_{n} b\left(y_{n}, a\right) w_{n} .
$$

Using boundedness of $\left\{y_{n}\right\}$ in $X_{k}$ and $\left\{w_{n}\right\}$ in $\ell_{1}$, summability of $\left\{\left|\lambda_{n}\right|\right\}$ and the definition of $b$, we see that the right-hand side of the first equality in the above display defines a continuous linear operators $T: X \rightarrow X$, taking values in $X_{k}$. Since the restriction $I+T_{\xi}$ of $I+T$ to $X_{k}$ is hypercyclic on $X_{k}, X_{k}$ is dense in $X$ and $X_{k}$ carries the topology stronger than the one inherited from $X$, we see that $I+T$ is hypercyclic (any hypercyclic vector for $I+T_{\xi}$ is also hypercyclic for $I+T$ ). Pick a hypercyclic vector $a$ for $I+S_{\xi}$. Since $a \neq 0$ and $b$ separates points of $\ell_{1}$, the functional $b(\cdot, a)$ is non-zero. Since $a$ is hypercyclic for $I+S_{\xi}$, we can pick a strictly increasing sequence $\left\{n_{k}\right\}_{k \in \mathbb{Z}_{+}}$of positive integers such that $\left\|\left(I+S_{\xi}\right)^{n} k_{k} a\right\|_{1} \leq 1$ for any $k \in \mathbb{Z}_{+}$. Then

$\left|b\left((I+T)^{n_{k}} x, a\right)\right|=\left|b\left(x,\left(I+S_{\xi}\right)^{n_{k}} a\right)\right| \leq p(x)\left\|\left(I+S_{\xi}\right)^{n_{k}} a\right\|_{1} \leq p(x)$ for any $\mathrm{x} \in \mathrm{X}$ and $\mathrm{k} \in \mathbb{Z}_{+}$.

Let $U=\{x \in X: p(x)<1\}$ and $V=\{x \in X:|b(x, a)|>1\}$. Clearly $U$ and $V$ are non-empty open subsets of $X(V \neq \varnothing$ since the functional $b(\cdot, a)$ is non-zero). Moreover, from the above display it follows that $(I+T)^{n} k(U) \cap V=\varnothing$ for each $k \in \mathbb{Z}_{+}$. Hence $I+T$ is non-mixing. Since $I+T$ is hypercyclic, the proof of Theorem 1.6 is complete.

\section{Proof of theorem 1.15}

Let $X$ be an infinite dimensional locally convex space, such that both $X$ and $X_{\beta}^{\prime}$ admit $\ell_{1}$-sequences with dense span. By Lemma 3.7, there exist sequences $\left\{x_{n}\right\}_{n \in \mathbb{Z}_{+}}$and $\left\{f_{n}\right\}_{n \in \mathbb{Z}_{+}}$in $X$ and $X^{\prime}$ respectively, satisfying (3.7.1-3.7.3). By Lemma 3.3, the closed balanced convex hulls $K$ and $D$ of $\left\{x_{n}: n \in \mathbb{Z}\right\}$ and $\left\{f_{n}: n \in \mathbb{Z}\right\}$ are Banach $s$-disks in $X$ and $X_{\beta}^{\prime}$ respectively. Moreover, $X_{K}$ is dense in $X$ and $X_{D}^{\prime}$ is dense in $X_{\beta}^{\prime}$. Since $D$ is $\beta$-compact, it is also $\sigma\left(X^{\prime}, X\right)$-compact. Hence the seminorm $p(x)=\sup \{|f(x)|: f \in D\}$ on $X$ is continuous with respect to the Mackey topology $\tau=\tau\left(X, X^{\prime}\right)$. Clearly each $\left|f_{n}\right|$ is bounded by 1 on $\{x \in X: p(x) \leq 1\}$ and therefore $\left\{f_{n}: n \in \mathbb{Z}\right\}$ is uniformly equicontinuous. By Lemma 3.7, we can assume that the same holds true if the original $\ell_{1}$-sequence in $X_{\beta}^{\prime}$ is uniformly equicontinuous. Assume for time being that either $X$ carries the Mackey topology $\tau$ or the original $\ell_{1}$-sequence in $X_{\beta}^{\prime}$ is uniformly equicontinuous. Then $\left\{f_{n}\right.$ : $n \in \mathbb{Z}\}$ is uniformly equicontinuous.

Consider the bilinear form on $X \times X^{\prime}$ defined by the formula $\beta(x, f)=f(x)$. Clearly $\beta$ separates points of $X$ and $X^{\prime}$ and $\beta$ is separately continuous on $X \times X_{\sigma}^{\prime}$. Since $X_{K}$ is dense in $X$ and $X_{D}^{\prime}$ is dense in $X_{\sigma}^{\prime}$, the bilinear form $b: X_{K} \times X_{D}^{\prime} \rightarrow \mathbb{K}$, being the restriction of $\beta$ to $X_{K} \times X_{D}^{\prime}$, separates points of $X_{K}$ and $X_{D}^{\prime}$. Moreover separate continuity of $\beta$ implies separate continuity of $b$ and therefore continuity of $b$ on $X_{K} \times X_{D}^{\prime}$ by means of the uniform boundedness principle (every separately continuous bilinear form on product of Banach spaces is continuous). Let $\mathcal{M}=X_{K} \widehat{\otimes}_{\pi} X_{D}^{\prime}$ be the projective tensor product of the Banach spaces $X_{K}$ and $X_{D}^{\prime}$ and $\mathcal{M}^{\mathcal{N}}$ be the closure in $\mathcal{M}$ of the set of $\xi \in X_{K} \otimes X_{D}^{\prime}$, for which the operator $T_{\xi}$ defined in (6.8) is nilpotent. By Proposition 6.7, the set $\left\{\xi \in \mathcal{M}^{\mathcal{N}}: I+T_{\xi}\right.$ and $I+S_{\xi}$ are both hypercyclic $\}$ is a dense $G_{\delta}$ subset of $\mathcal{M}^{\mathcal{N}}$. In particular, we can pick $\xi \in \mathcal{M}^{\mathcal{N}} \subset X_{D} \widehat{\otimes}_{\pi} \ell_{1}$ such that $I+T_{\xi}$ and $I+S_{\xi}$ are hypercyclic. Using once again the theorem characterizing the shape of elements of the projective tensor product, we see that there exist bounded sequences $\left\{y_{n}\right\}_{n \in \mathbb{Z}_{+}}$and $\left\{g_{n}\right\}_{n \in \mathbb{Z}_{+}}$in $X_{K}$ and $X_{D}^{\prime}$ respectively and $\lambda \in \ell_{1}$ such that $\xi=\sum_{n=0}^{\infty} \lambda_{n} y_{n} \otimes g_{n}$. Then the operators $T_{\xi}$ and $S_{\xi}$ act according to the following formulae on $X_{K}$ and $X_{D}^{\prime}$ respectively:

$$
T_{\xi} x=\sum_{n=0}^{\infty} \lambda_{n} g_{n}(x) y_{n}, \text { and } S_{\xi} f=\sum_{n=0}^{\infty} \lambda_{n} f\left(y_{n}\right) g_{n},
$$

where we used the specific shape of our bilinear form. Using boundedness of $\left\{y_{n}\right\}$ in $X_{K}$ and $\left\{g_{n}\right\}$ in $X_{D}^{\prime}$ and summability of $\left\{\left|\lambda_{n}\right|\right\}$, we see that the right-hand sides of the equalities in the above display define linear operators $T$ and $S$ on $X$ and $X^{\prime}$, taking values in $X_{K}$ and $X_{D}^{\prime}$ respectively. Since $\left\{f_{n}: n \in \mathbb{Z}_{+}\right\}$is uniformly equicontinuous and $\left\{g_{n}\right\}$ is bounded in $X_{D}^{\prime},\left\{g_{n}: n \in \mathbb{Z}_{+}\right\}$is also uniformly equicontinuous. It follows that $T$ is continuous as an operator from $X$ to $X_{K}$ and therefore $T \in L(X)$. It is also easy to verify that $S=T^{\prime}$ and therefore $S \in L\left(X_{\beta}^{\prime}\right)$. Since the restriction $I+T_{\xi}$ of $I+T$ to $X_{K}$ is hypercyclic on $X_{K}, X_{K}$ is dense in $X$ and $X_{K}$ carries the topology stronger than the one inherited from $X$, we see that $I+T$ is hypercyclic (any hypercyclic vector for $I+T_{\xi}$ is also hypercyclic for $I+T)$. Similarly $I+S=(I+T)^{\prime}$ is hypercyclic on $X_{\beta}^{\prime}$ (any hypercyclic vector for $I+S_{\xi}$ is also hypercyclic for $I+S$ ). Hence $I+T$ is a dual hypercyclic operator. In order to complete the proof of Theorem 1.15, it remains to consider the case when $X$ carries the weak topology $\sigma=\sigma\left(X, X^{\prime}\right)$. By the already proven part of Theorem 1.15, there is a dual hypercyclic operator $R$ on $X_{\tau}$. Since $L\left(X_{\tau}\right)=L\left(X_{\sigma}\right), R \in$ $L(X)$. Since $\tau$ is stronger than $\sigma, R$ is hypercyclic on $X=X_{\sigma}$. Hence $R$ is dual hypercyclic on $X$. The proof of Theorem 1.15 is complete.

\section{Generic bilateral weighted shifts}

For each $w \in \ell_{\infty}(\mathbb{Z}), T_{w}$ stands for the bounded linear operator acting on $\ell_{p}(\mathbb{Z}), 1 \leq p<\infty$ or $c_{0}(\mathbb{Z})$, defined on the canonical basis $\left\{e_{n}\right\}_{n \in \mathbb{Z}}$ by

$$
T_{w} e_{n}=w_{n} e_{n-1} \quad \text { for } n \in \mathbb{Z} .
$$

If additionally $w_{n} \neq 0$ for each $n \in \mathbb{Z}$, the operator $T_{w}$ is called the bilateral weighted shift with weight sequence $w$. Cyclic properties of bilateral weighted shifts have been intensely studied. Hypercyclicity and supercyclicity of bilateral weighted shifts were characterized by Salas [12,31] in terms of the weight sequences. It was observed in [37], Proposition 5.1 that the Salas conditions admit a simpler equivalent form:

Theorem B Let $T$ be a bilateral weighted shift with weight sequence $w$ acting on $\ell_{p}(\mathbb{Z})$ with $1 \leq p<\infty$ or $c_{0}(\mathbb{Z})$. Then $T$ is hypercyclic if and only iffor any $m \in \mathbb{Z}_{+}$,

$$
\lim _{\rightarrow \infty} \max \{\tilde{w}(m-n+1, m),(\tilde{w}(m+1, m+n)) \quad\}=0
$$

and $T$ is supercyclic if and only if for any $m \in \mathbb{Z}_{+}$,

$$
\lim _{n \rightarrow+\infty} \tilde{w}(m-n+1, m) \tilde{w}(m+1, m+n)^{-1}=0,
$$


where $\tilde{w}(a, b)=\prod_{j=a}^{b}\left|w_{j}\right|$ for $a, b \in \mathbb{Z}$ with $a \leq b$.

We address the issue of hypercyclicity and supercyclicity of generic bilateral weighted shifts in the Baire category sense. For each $c>0$ let

$B_{c}=\left\{w \in \ell_{\infty}(\mathbb{Z}):\|w\|_{\infty} \leq c\right\}$.

Clearly $B_{c}$ endowed with the coordinatewise convergence topology is a compact metrizable topological space.

Theorem 6.8 Let $1 \leq p<\infty$. For each $c>1$ the set of $w \in B_{c}$ for which $T_{w}$ acting on $\ell_{p}(\mathbb{Z})$ is hypercyclic is a dense $G_{\delta}$ subset of $B_{c}$. For each $c>$ 0 the set of $w \in B_{c}$ for which $T_{w}$ is supercyclic and $I+T_{w}$ is hypercyclic is a dense $G_{\delta}$-subset of $B_{c}$.

It is worth noting that if $w_{n}=0$ for some $n \in \mathbb{Z}$ then the range of the operator $T_{w}$ is not dense and therefore $T_{w}$ can not be supercyclic. Thus any $T_{w}$ for $w$ from the dense $G_{\delta}$-sets in the above theorem are indeed bilateral weighted shifts. Recall that $T_{w}$ is compact if and only if $w \in c_{0}(\mathbb{Z})$. For compact bilateral weighted shifts we can replace the coordinatewise convergence topology on the space of weights by stronger topologies.

Theorem 6.9 Let $1 \leq p<\infty$. Let $E$ be a linear subspace of $c_{0}(\mathbb{Z})$ carrying its own $\mathcal{F}$-space topology stronger than the one inherited from $c_{0}(\mathbb{Z})$ and such that the space $\psi(\mathbb{Z})$ of sequences with finite support is densely contained in $E$. Then the set of $w \in E$ for which $T_{w}$ acting on $\ell_{p}(\mathbb{Z})$ is supercyclic and $I+T_{w}$ is hypercyclic is a dense $G_{\delta}$-subset of $E$.

Remark 6.10 As a corollary of the above theorem we obtain that the set of weights $w \in c_{0}(\mathbb{Z})$ for which $T_{w}$ acting on $\ell_{p}(\mathbb{Z})$ is supercyclic and $I+T_{w}$ is hypercyclic is a dense $G_{\delta}$-subset of the Banach space $c_{0}(\mathbb{Z})$.

It is easy to see that the dual of $T_{w}$ acting on $\ell_{p}(\mathbb{Z})$ for $1<p<\infty$ acts on $\ell_{q}(\mathbb{Z})$ with $\frac{1}{p}+\frac{1}{q}=1$ according to the formula

$T_{w}^{\prime} e_{n}=w_{n+1} e_{n+1} \quad$ for $n \in \mathbb{Z}$.

Considering the isometry $U$ on $\ell_{q}(\mathbb{Z})$ defined by $U e_{n}=e_{-n}$ for each $n \in \mathbb{Z}$, we see that $U^{-1} T_{w}^{\prime} U=T_{w^{\prime}}$, where $w_{n}^{\prime}=w_{1-n}$ for any $n \in \mathbb{Z}$. Thus $T_{w}^{\prime}$ is hypercyclic or supercyclic if and only if , (acting on $\left.\ell_{q}(\mathbb{Z})\right)$ is. In the case $p=2$, the Hilbert space adjoint $T_{w}^{*}$ acts on $\ell_{2}(\mathbb{Z})$ in a similar way $T_{w}^{*} e_{n}=\overline{w_{n+1}} e_{n+1}$ and is unitarily similar to $T_{w}^{\prime}$ with a diagonal unitary operator providing the similarity. Thus the cyclicity properties of $T_{w}^{\prime}$ and $T_{w}^{*}$ are the same.

Taking into account the fact that the map $w \mapsto w^{\prime}$ is a homeomorphism of $B$ onto itself for each $c>0$, we immediately obtain the following corollary of Theorem 6.8.

Corollary 6.11 For each $c>1$ the set of $w \in B_{c}$ for which both $T_{w}$ and $T_{w}^{\prime}$ acting on $\ell_{2}(\mathbb{Z})$ are hypercyclic is a dense $G_{\delta}$-subset of $B_{c}$. For each $c>$ 0 the set of $w \in B_{c}$ for which both $T_{w}$ and $T_{w}^{\prime}$ are supercyclic and both $I+$ $T_{w}$ and $I+T_{w}^{\prime}$ are hypercyclic is a dense $G_{\delta}$-subset of $B_{c}$.

Similarly the next corollary follows from Theorem 6.9.

Corollary 6.12 Suppose that the space E from Theorem 6.9 satisfies the additional symmetry condition that $(J x)_{n}=x_{k-n}$ is an invertible continuous linear operator on $E$ for some $k \in \mathbb{Z}$. Then set of $w \in E$ for which both $T_{w}$ and $T_{w}^{\prime}$ acting on $\ell_{2}(\mathbb{Z})$ are supercyclic and both $I+T_{w}$ and $I+T_{w}^{\prime}$ are hypercyclic is a dense $G_{\delta}$-subset of $E$.
Applying the above corollary to weighted $c_{0}$-spaces with symmetric weight sequence yields the following result.

Corollary 6.13 Let $\left\{a_{n}\right\}_{n \in \mathbb{Z}_{+}}$be any sequence of positive numbers. Then there exists $w \in c_{0}(\mathbb{Z})$ such that $\left|w_{n}\right| \leq a_{|n|}$ for each $n \in \mathbb{Z}, T_{w}$ and $T_{w}^{\prime}$ acting on $\ell_{2}(\mathbb{Z})$ are supercyclic and $I+T_{w}$ and $I+T_{w}^{\prime}$ are hypercyclic.

We conclude this section by proving Theorems 6.8 and 6.9.

Proof of Theorem 6.8. It is straightforward to verify that the maps $w \mapsto T_{w}$ and $w \mapsto I+T_{w}$ from $\Omega=B_{c}$ into $L\left(\ell_{p}(\mathbb{Z})\right.$ )satisfy (6.3). Pick a non-empty open subset $U$ of $B_{c}$ and non-empty open subsets $V$ and $W$ of $\ell_{p}(\mathbb{Z})$.

Case $c>1$ : By definition of the topology of $B_{c}$, there exist $w \in U$ and a positive integer $m$ such that $w_{k}=c$ for $k>m, w_{m}=c^{-1}$ for $k<-m$ and $w_{k} \neq 0$ for $-m \leq k \leq m$. According to Theorem $\mathrm{B}$, the bilateral weighted shift $T_{w}$ is hypercyclic. Hence we can choose $x \in V$ and $n \in \mathbb{Z}_{+}$ such that $T_{w}^{n} x \in W$. Thus $\left(w, x, T_{w}^{n} x\right) \in U \times V \times W$ and therefore (6.4) is satisfied. By Theorem 6.2, the set of $w \in B_{c}$ for which $T_{w}$ is hypercyclic is a dense $G_{\delta}$-subset of $B_{c}$.

Case $c>0$ : As above, there exist $w \in U$ and a positive integer $m$ such that $w_{k}=c$ for $k>m, w_{k}=c / 2$ for $k<-m$ and $w_{k} \neq 0$ for $-m \leq k \leq m$. According to Theorem $\mathrm{B}$, the bilateral weighted shift $T_{w}$ is supercyclic. Hence we can choose $x \in V, n \in \mathbb{Z} \mathbb{Z}$, and $\lambda \in \mathbb{K}$ such that $\lambda T_{w}^{n} x \in W$. Thus $\left(w, x, \lambda T_{w}^{n} x\right) \in U \times V \times W$ and therefore (6.6) is satisfied. By Theorem 6.3 , the set of $w \in B_{c}$ for which $T_{w}$ is supercyclic is a dense $G_{\delta}$-subset of $B_{c}$.

Finally, we can pick $m \in \mathbb{Z}, w \in U, x \in V$, and $y \in W$, such that $w_{m}=$ $0, w_{m} \neq 0$ for $k>m, x$ and $y$ have finite supports and $x_{k}=y_{k}=0$ for $k$ $>m$. It is straight forward to check that

$$
x, y \in \operatorname{span}\left\{e_{m}, e_{m+1}, \ldots\right\} \subseteq \operatorname{ker}^{\dagger} T_{w} .
$$

By Corollary 6.6, the set of $w \in B_{c}$ for which $I+T_{w}$ is hypercyclic is a dense $G_{\delta}$-subset of $B_{c}$.

Proof of Theorem 6.9. We use the same notation as in the proof of Theorem 6.8. Since $\varphi(\mathbb{Z})$ is dense in $\ell_{p}(\mathbb{Z})$ and in $E$, we can choose $w^{\prime} \in U, x^{\prime} \in V, \quad y^{\prime} \in W$ and a positive integer $m$ such that $x_{k}^{\prime}=y_{k}^{\prime}=w_{k}^{\prime}=0$ if $|k|>m$. Choosing positive numbers $\varepsilon_{n}$ for $n>-m$ small enough, we can ensure that the series $\sum_{n=m+1}^{\infty} \varepsilon_{n} e_{n}$ converges in $E$ to $w^{\prime \prime} \in E$, with $w=w^{\prime}+w^{\prime \prime} \in U$ and $w_{k}=w_{k}^{\prime}+w_{k}^{\prime \prime} \neq 0$ for $k>-m$. Clearly $F=\left\{u \in \ell_{p}(\mathbb{Z}): u_{k}=0\right.$ for $\left.k<-m\right\}$ is a closed invariant subspace of $T_{w}$ and $x, y \in F$. Moreover, the restriction $R$ of $T_{w}$ to $F$ is isometrically similar to the backward weighted shift on $\ell_{p}$ with weight sequence $\left\{w_{-m}, w_{-m+1}, \ldots\right\}$ By the Hilden and Wallen theorem [30] and the Salas theorem [12], $R$ is supercyclic and $I+R$ is hypercyclic. Since $x^{\prime} \in V \cap F$ and $y^{\prime} \in W \cap F$ we see that the open subsets $V \cap F$ and $W \cap F$ of $F$ are non-empty. Since $R$ is supercyclic, we can choose $x \in V \cap F, n \in \mathbb{Z}_{+}$and $\lambda \in \mathbb{K}$ such that $\lambda R^{n} x=\lambda T_{w}^{n} x \in W \cap F$. Thus $\left(w, x, \lambda T_{w}^{n} x\right) \in U \times V \times W$ and therefore (6.6) is satisfied. By Theorem 6.3, the set of $w \in E$ for which $T_{w}$ is supercyclic is a dense $G_{\delta}$-subset of $E$. Finally, since $I$ $+R$ is hypercyclic, we can choose $x \in V \cap F$ and $n \in \mathbb{Z}_{+}$such that $(I+R)^{n} x=\left(I+T_{w}\right)^{n} x \in W \cap F$. Thus $\left(w, x,\left(I+T_{w}\right)^{n} x\right) \in U \times V \times W$ and therefore (6.4) is satisfied for the map $w \mapsto I+T_{w}$. By Theorem 6.2, 
the set of $w \in E$ for which $I+T_{w}$ is hypercyclic is a dense $G_{\delta}$-subset of $E$.

\section{Mixing Operators on Spaces with Weak Topology}

In this section we shall prove Theorem 1.8. In order to do so we need a characterization of linear maps with no non-trivial finite dimensional invariant subspaces. The underlying field plays no role in this linear algebraic statement, so, for sake of generality we formulate and prove it for linear maps on linear spaces over an arbitrary field.

\section{Linear maps without finite dimensional invariant subspaces}

Throughout this subsection $k$ is a field, $X$ is a linear space over $k$, $T: X \rightarrow X$ is a $k$-linear map, $\mathcal{P}=k[z]$ is the space of polynomials on one variable, $\mathcal{P}=k(z)$ is the space of rational functions and $M$ is the operator on $\mathcal{P}$ of multiplication by the argument:

$$
M: \mathcal{R} \rightarrow \mathcal{R}, \quad M f(z)=z f(z) .
$$

We denote $\mathcal{P}^{*}=\mathcal{P} \backslash\{0\}$ and consider the degree function $\operatorname{deg}: \mathcal{R} \rightarrow \mathbb{Z} \cup\{-\infty\}$, extending the conventional degree of a polynomial. We set $\operatorname{deg}(0)=-\infty$ and let $\operatorname{deg}(p / q)=\operatorname{deg} p-\operatorname{deg} q$, where $P$ and $q$ are non-zero polynomials and the degrees in the right hand side are the conventional degrees of polynomials. Clearly this function is well-defined and is a grading on $\mathcal{P}$, that is, it satisfies the properties:

(d1) $\operatorname{deg}\left(r_{1} r_{2}\right)=\operatorname{deg}\left(r_{1}\right)+\operatorname{deg}\left(r_{2}\right)$ for any $r_{1}, r_{2} \in \mathcal{R} ;$

(d2) $\operatorname{deg}\left(r_{1}+r_{2}\right) \leq \max \left\{\operatorname{deg} r_{1}, \operatorname{deg} r_{2}\right\}$ for any $r_{1}, r_{2} \in \mathcal{R}$;

$(d \#)$ if $r_{1}, r_{2} \in \mathcal{R}$ and $\operatorname{deg} r_{1} \neq \operatorname{deg} r_{2}$, then $\operatorname{deg}\left(r_{1}+r_{2}\right)=\max \left\{\operatorname{deg} r_{1}, \operatorname{deg} r_{2}\right\}$.

Note that if $p$ is a non-zero polynomial, then $\operatorname{deg} P$ is the usual degree of $P$.

As usual, a linear subspace $E$ of $X$ is called $T$ - invariant if $T(E) \subseteq E$ and it is called $T$ - biinvariant if $T(E) \subseteq E$ and $T^{-1}(E) \subseteq E$. The following lemma is a key ingredient in the proof of Theorem 1.8.

Lemma 7.1 Let $T$ be a linear operator on a linear space $X$ with no non-trivial finite dimensional invariant subspaces and let $L$ be a finite dimensional subspace of $X$. Then there exists $n_{0}=n_{0}(L) \in \mathbb{N}$ such that $p(T)(L) \cap L=\{0\}$ for any $p \in \mathcal{P}$ with $\operatorname{deg} p \geq n_{0}$.

In order to prove the above lemma, we need some preparation.

Lemma 7.2 Let $T$ be a linear operator on a linear space X. Then $T$ has no non-trivial finite dimensional invariant subspaces if and only if $p(T)$ is injective for any non-zero polynomial $P$.

Proof. If $p$ is a non-zero polynomial and $p(T)$ is non-injective, then there is non-zero $x \in X$ such that $p(T) x=0$. Let $k=\operatorname{deg} p$. It is straightforward to verify that $E=\operatorname{span}\left\{x, T x, \ldots, T^{k-1} x\right\}$ is a nontrivial finite dimensional invariant subspace for $T$. Assume now that $T$ has a non-trivial finite dimensional invariant subspac $L$. Let $p$ be the characteristic polynomial of the restriction of $T$ to $L$. By the HamiltonCayley theorem $p(T)$ vanishes on $L$. Hence $p(T)$ is non-injective.

Definition 7.3 For a linear operator $T$ on a linear space $X$ we say that vectors $x_{1}, \ldots, x_{n}$ in $X$ are $T$-independent if for any polynomials $p_{1}, \ldots, p_{n}$, the equality $\sum_{j=1}^{n} p_{j}(T) x_{j}=0$ implies $p_{j}=0$ for $1 \leq j \leq n$. Otherwise, we say that $x_{1}, \ldots, x_{n}$ are $T$ - dependent. A set $A \subset X$ is called $T$ - independent if any pairwise different vectors $x_{1}, \ldots, x_{n} \in A$ are $T$ independent.

For any non-zero $x \in X$, we define

$$
F(T, x)=\left\{y \in X \text { : there are } \mathrm{p} \in \mathcal{P}^{*} \text { and } \mathrm{q} \in \mathcal{P} \text { such that } p(T) y=q(T) x\right\} .
$$

Lemma 7.4 Let $T$ be a linear operator on a linear space $X, x \in X \backslash\{0\}$ and $F(T, x)$ be the space defined in (29). Then $F(T, x)$ is a T-biinvariant linear subspace of $X$.

Proof. Let $y, u \in F(T, x)$ and $t, s \in k$. Then we can pick $p_{1}, p_{2} \in \mathcal{P}^{*}$ and $q_{1}, q_{2} \in \mathcal{P}$ such that $p_{1}(T) y=q_{1}(T) x$ and $p_{2}(T) u=q_{2}(T) x$. Hence $\left(p_{1} p_{2}\right)(T)(t y+s u)=\left(t q_{1} p_{2}+s q_{2} p_{1}\right)(T) x$. Since $p_{1}$ and $p_{2}$ are non-zero, the polynomial $p_{1} p_{2}$ is also non-zero and we have $t y+s u \in F(T, x)$ and therefore $F(T, x)$ is a linear subspace of $X$. Clearly $p_{1}(T)(T y)=\tilde{q}_{1}(T) x$, where $\tilde{q}_{1}(z)=z q(z)$. Hence $T y \in F(T, x)$, which proves the $T$-invariance of $F(T, x)$. Assume now that $w \in X$ and $T w \in F(T, x)$. Thus we can pick $p_{3} \in \mathcal{P}^{*}$ and $q_{3} \in \mathcal{P}$ such that $p_{3}(T) T w=q_{3}(T) x$. Hence $\tilde{p}_{3}(T) w=q_{3}(T) x$, where $\tilde{p}_{3}(z)=z p_{3}(z)$, and therefore $w \in F(T, x)$. That is, $F(T, x)$ is $T$-biinvariant.

By the above lemma, we can consider linear operators, being restrictions of $T$ to the invariant subspaces $F(T, x)$. The following lemma describes these restrictions in the case when $T$ has no nontrivial finite dimensional invariant subspaces.

Lemma 7.5 Let $T$ be a linear operator on a linear space $X$ with no non-trivial finite dimensional invariant subspaces. Let also $x \in X \backslash\{0\}$ and $F(T, x)$ be defined in (7.1). For each $y \in F(T, x)$ and any $p \in \mathcal{P}^{*}$, $q \in \mathcal{P}$ satisfying $p(T) y=q(T) x$, we write $r_{x, y}=q / p$. Then the rational function $r_{x, y}$ does not depend on the choice of $p \in \mathcal{P}^{*}$ and $q \in \mathcal{P}$ satisfying $p(T) y=q(T) x$, the map $S_{x} y=r_{x, y}$ from $F(T, x)$ to $\mathcal{P}$ is linear and $S_{x} T y=M S_{x} y$ for any $y \in F(T, x)$.

Proof. Let $y \in F(T, x)$ and $p, p_{1} \in \mathcal{P}^{*}, q, q_{1} \in \mathcal{P}$ be such that $p(T) y=q(T) x$ and $p_{1}(T) y=q_{1}(T) x$. Hence $\left(p_{1} p\right)(T) y=\left(q_{1} p\right)(T) x=\left(q p_{1}\right)(T) x$ and therefore $\left(q_{1} p-q p_{1}\right)(T) x=0$. Since $x \neq 0$, Lemma 7.2 implies that $q_{1} p=q p_{1}$, or equivalently, $q / p=q_{1} / p_{1}$. Thus $q / p$ does not depend on the choice of $p \in \mathcal{P}^{*}$ and $q \in \mathcal{P}$ satisfying $p(T) y=q(T) x$. In particular, the map $y \mapsto r_{x, y}$ from $F(T, x)$ to $\mathcal{P}$ is well defined. Next, let $y, u \in F(T, x)$ and $t, s \in k$. Then we can pick $p_{1}, p_{2} \in \mathcal{P}^{*}$ and $q_{1}, q_{2} \in \mathcal{P}$ such that $p_{1}(T) y=q_{1}(T) x$ and $p_{2}(T) u=q_{2}(T) x$. Hence $\left(p_{1} p_{2}\right)(T)(t y+s u)=\left(t q_{1} p_{2}+s q_{2} p_{1}\right)(T) x$. It follows that

$$
r_{x, t y+s u}=\frac{t q_{1} p_{2}+s q_{2} p_{1}}{p_{1} p_{2}}=t \frac{q_{1}}{p_{1}}+s \frac{q_{2}}{p_{2}}=t r_{x, y}+s r_{x, u}
$$

and the linearity of the map $y \mapsto r_{x, y}$ is also verified. It remains to show that $S_{x} T y=M S_{x} y$. Clearly $S_{x} y=q_{1} / p_{1}$. Since $p_{1}(T)(T y)=T q_{1}(T) x$, we have $S_{x} T y(z)=z q_{1}(z) / p_{1}(z)$. Hence $S_{x} T y=M S_{x} y$.

Lemma 7.6 Let $T$ be a linear operator with no non-trivial finite dimensional invariant subspaces acting on a linear space $X$ and $A$ be a $T$ -independent subset of $X$. Then for each $x \in A$,

$$
F(T, x) \cap \operatorname{span}\left(\bigcup_{y \in A \backslash\{x\}} F(T, y)\right)=\{0\},
$$

where the spaces $F(T, u)$ are defined in (7.1). 
Proof. Assume that the intersection in the above display contains a non-zero vector $u$. Since $u \in F(T, x)$, there exist $p \in \mathcal{P}^{*}$ and $q \in \mathcal{P}$ such that $p(T) u=q(T) x$. Since $u \neq 0$ and $p \neq 0$, according to Lemma 7.2, $p(T) u \neq 0$. It follows that $q \neq 0$. On the other hand, since $u$ is a non-zero element of the span of the union of $F(T, Y)$ for $y \in A \backslash\{x\}$, there exist pairwise different $x_{1}, \ldots, x_{n} \in A \backslash\{x\}$ and $u_{j} \in F\left(T, x_{j}\right) \backslash\{0\}$ such that $u=u_{1}+\ldots+u_{n}$. Pick $p_{j} \in \mathcal{P}^{*}$ and $q_{j} \in \mathcal{P}$ for which $p_{j}(T) u_{j}=q_{j}(T) x_{j}$ for $1 \leq j \leq n$. Since $u_{j} \neq 0$ and $p_{j} \neq 0$, from Lemma 7.2 it follows that $p_{j}(T) u_{j} \neq 0$ and therefore $q_{j} \neq 0$. Consider the polynomials $p_{0}=p p_{1} \ldots p_{n}, \tilde{p}_{0}=p_{0} / p, \tilde{p}_{j}=p_{0} / p_{j}$ for $1 \leq j \leq n$. Then

$$
p_{0}(T) u=\left(q \tilde{p}_{0}\right)(T) x \text { and } p_{0}(T) u_{j}=\left(q_{j} \tilde{p}_{j}\right)(T) x_{j} \text { for } 1 \leq \mathrm{j} \leq \mathrm{n} .
$$

Taking into account that $u=u_{1}+\ldots+u_{n}$, we obtain

$$
\left(q \tilde{p}_{0}\right)(T) x=\sum_{j=1}^{n}\left(q_{j} \tilde{p}_{j}\right)(T) x_{j} .
$$

Since the polynomials $q \tilde{p}_{0}$ and $q_{j} \tilde{p}_{j}$ are non-zero, the last display contradicts the $T$-independence of $A$, since $x, x_{1}, \ldots, x_{n}$ are pairwise different elements of $A$.

Proof of Lemma 7.1. Clearly there exists in $L$ a maximal $T$ -independent subset $A=\left\{x_{1}, \ldots, x_{k}\right\}$ (since $T$-independence implies linear independence and $L$ is finite dimensional, $A$ is a finite set). It follows from the maximality of $A$ that $L$ is contained in the sum of $F\left(T, x_{j}\right)$ for $1 \leq j \leq k$. The last sum is direct according to Lemma 7.6:

$$
L \subseteq N=\bigoplus_{=1} F(T, x)
$$

Thus any $x \in N$ can be uniquely presented as a sum $u_{1}^{x}+\ldots+u_{k}^{x}$, where $u_{j}^{x} \in F\left(T, x_{j}\right)$. Using Lemmas 7.5 and 7.4 , we can consider the linear operator

$$
R: N \rightarrow \mathcal{R}^{k}, \quad R(x)=\left(R_{1} x, \ldots, R_{k} x\right), \text { where } R_{j} x=S_{x_{j}} u_{j}^{x} \text { for } 1 \leq j \leq k .
$$

According to Lemmas 7.4 and 7.5 we also have that $N$ is a $T$-biinvariant subspace of $X$ and $R(T x)_{j}=M(R x)_{j}$ for $1 \leq j \leq k$. For each $x \in N$, let

$$
(x)=\max _{\leq j \leq k} \operatorname{deg} R x .
$$

Clearly $\delta(0)=-\infty$ and $\delta(x) \in \mathbb{Z}$ for each $x \in N \backslash\{0\}$. Let also

$$
\Delta^{+}=\sup _{x \in L} \delta(x) \text { and } \Delta^{-}=\inf _{x \in L \backslash\{0\}} \delta(x) .
$$

Using the fact that $L$ is finite dimensional, we will show that $\Delta^{+}$and $\Delta^{-}$are finite. Indeed, assume that either $\Delta^{+}=+\infty$ or $\Delta^{-}=-\infty$. Then there exists a sequence $\left\{u_{l}\right\}_{l \in \mathbb{N}}$ of non-zero elements of $L$ such that the sequence $\left\{\delta\left(u_{l}\right)\right\}_{l \in \mathbb{N}}$ is strictly monotonic. For each $l$ we can pick $j(l) \in\{1, \ldots, k\}$ such that $\delta\left(u_{l}\right)=\operatorname{deg} R_{j(l)} u_{l}$. Then there is $v \in\{1, \ldots, k\}$ such that the set $B_{v}=\{l \in \mathbb{N}: j(l)=v\}$ is infinite. It follows that the degrees $\operatorname{deg} R_{v} u_{l}$ for $l \in B_{v}$ are pairwise different. Property (d3) of the degree function implies that the rational functions $R_{v} u_{l}$ for $l \in B_{v}$ are linearly independent. Hence the infinite set $\left\{u_{l}: l \in B_{v}\right\}$ is linearly independent in $X$, which is impossible since all $u_{1}$ belong to the finite dimensional space $L$. Thus $\Delta^{+}$and $\Delta^{-}$are finite.
Now let $p \in \mathcal{P}^{*}$ and $m=\operatorname{deg} p$. From (d1) and the equality $R_{j} T x=M R_{j} x$, we immediately get that $\delta(p(T) x)=\delta(x)+m$ for each $x \in N$. Therefore, $\inf \{\delta(x): x \in p(T)(L) \backslash\{0\}\}=\Delta^{-}+m$. In particular, if $m>\Delta^{+}-\Delta^{-}$, then

$$
\inf _{x \in p(T)(L) \backslash\{0\}} \delta(x)=\Delta^{-}+m>\Delta^{+}=\sup _{x \in L} \delta(x) .
$$

Thus $\delta(u)>\delta(v)$ for any non-zero $u \in P(T)(L)$ and $v \in L$, which implies that $p(T)(L) \cap L=\{0\}$ whenever $\operatorname{deg} p>\Delta^{+}-\Delta^{-}$. Thus the number $n_{0}=\Delta^{+}-\Delta^{-}+1$ satisfies the desired condition.

\section{Proof of Theorem 1.8}

The implications $(1.8 .4) \Rightarrow(1.8 .3) \Rightarrow(1.8 .2)$ are trivial. Assume that $T$ is transitive and $T^{\prime}$ has a non-trivial finite dimensional invariant subspace. Then $T$ has a non-trivial closed invariant subspace of finite codimension. Passing to the quotient by this subspace, we obtain a transitive operator on a finite dimensional topological vector space. Since there is only one Hausdorfflinear topology on a finite dimensional space, we arrive to a transitive operator on a finite dimensional Banach space. Since transitivity and hypercyclicity for operators on separable Banach spaces are equivalent [3], we obatin a hypercyclic operator on a finite dimensional Banach space. On the other hand, it is well known that such operators do not exist, see, for instance, [7]. This proves the implication $(1.8 .2) \Rightarrow(1.8 .1)$. It remains to show that (1.8.1) implies (1.8.4).

Assume that (1.8.1) is satisfied and (1.8.4) fails. Then there exist non-empty open subsets $U$ and $V$ of $X$ and a sequence $\left\{p_{l}\right\}_{l \in \mathbb{Z}_{+}}$of polynomials such that $\operatorname{deg} p_{l} \rightarrow \infty$ and $p_{l}(T)(V) \cap U=\varnothing$ for each $l \in \mathbb{Z}_{+}$. Since $X$ carries weak topology, there exist two finite linearly independent sets $\left\{f_{1}, \ldots, f_{n}\right\}$ and $\left\{g_{1}, \ldots, g_{m}\right\}$ in $X^{\prime}$ and two vectors $\left(a_{1}, \ldots, a_{n}\right) \in \mathbb{K}^{n}$ and $\left(b_{1}, \ldots, b_{m}\right) \in \mathbb{K}^{m}$ such that $U_{0} \subseteq U$ and $V_{0} \subseteq V$, where

$U_{0}=\left\{u \in X: f_{j}(u)=a_{j}\right.$ for $\left.1 \leq j \leq n\right\}$ and $V_{0}=\left\{u \in X: g_{j}(u)=b_{j}\right.$ for $\left.1 \leq j \leq m\right\}$.

Let $L=\operatorname{span}\left\{f_{1}, \ldots, f_{n}, g_{1}, \ldots, g_{m}\right\}$. Since $T^{\prime}$ has non non-trivial finite dimensional invariant subspaces, by Lemma 7.1, for any sufficiently large $l, p_{l}\left(T^{\prime}\right)(L) \cap L=\{0\}$. For such an $l$, the equality $p_{l}\left(T^{\prime}\right)(L) \cap L=\{0\}$ together with the injectivity of $p_{l}\left(T^{\prime}\right)$, provided by Lemma 7.2, and the definition of $L$ imply that the vectors $p_{l}\left(T^{\prime}\right) f_{1}, \ldots, p_{l}\left(T^{\prime}\right) f_{n}, g_{1}, \ldots, g_{m}$ are linearly independent. Hence there exists a vector $u \in X$ such that

$$
p_{l}\left(T^{\prime}\right) f_{j}(u)=a_{j} \text { for } 1 \leq j \leq n \text { and } g_{j}(u)=b_{j} \text { for } 1 \leq j \leq m \text {. }
$$

Since $p_{l}\left(T^{\prime}\right) f_{j}(u)=f_{j}\left(p_{l}(T) u\right)$, the last display implies that $u \in V_{0} \subseteq V$ and $p_{l}(T) u \in U_{0} \subseteq U$. Hence $p_{l}(T)(V) \cap U$ contains $p_{l}(T) u$ and therefore is non-empty. This contradiction completes the proof.

\section{Spaces without Supercyclic Semigroups $\left\{T_{t}\right\}_{t \in \mathbb{R}_{+}}$}

We shall prove Theorem 1.7 and show that on certain topological vector spaces there are no strongly continuous supercyclic semigroups $\left\{T_{t}\right\}_{t \in \mathbb{R}_{+}}$. In this section by the dimension $\operatorname{dim} X$ of a vector space $X$ we mean its algebraic dimension (=the cardinality of the Hamel basis). Symbol $c$ stands for the cardinality of continuum: $c=2 \aleph^{N_{0}}$. The next theorem is the main result of this section. 
Theorem 8.1 Let $X$ be an infinite dimensional locally convex space such that either in $X$ or in $X_{\sigma}^{\prime}$ there are no compact metrizable subsets whose linear span has dimension $c$. Then there are no strongly continuous supercyclic semigroups $\left\{T_{t}\right\}_{t \in \mathbb{R}_{+}}$on $X$.

The above theorem immediately implies the following corollary.

Corollary 8.2 Let $X$ be an infinite dimensional locally convex space such that $\operatorname{dim} X<\mathcal{c}$ or $\operatorname{dim} X^{\prime}<\mathcal{c}$. Then there are no strongly continuous supercyclic semigroups $\left\{T_{t}\right\}_{t \in \mathbb{R}_{+}}$on $X$.

We prove Theorem 8.1 at the end of this section. First, we shall prove Theorem 1.7 by means of application of Theorems 1.8 and 8.1 .

Proof of Theorem1.7.Let $T \in L(\omega)$ be such that $T^{\prime}$ has no nontrivial finite dimensional invariant subspaces and $\left\{p_{l}\right\}_{l \in \mathbb{Z}_{+}}$be a sequence of polynomials such that $\operatorname{deg} p_{l} \rightarrow \infty$ as $l \rightarrow \infty$. Since the topology of $\omega$ is weak, Theorem 1.8 implies that for each non-empty open subsets $U$ and $V$ of $\omega, p_{l}(U) \cap V \neq \varnothing$ for all sufficiently large l. Hence $\left\{\left(x, p_{l}(T) x\right): x \in \omega, l \in \mathbb{Z}_{+}\right\}$is dense in $\omega \times \omega$. By Theorem $\mathrm{U},\left\{p_{l}(T): l \in \mathbb{Z}_{+}\right\}$is universal. It remains to show that there are no strongly continuous supercyclic semigroups $\left\{T_{t}\right\}_{t \in \mathbb{R}_{+}}$on $X$. Recall that $\omega^{\prime}=\varphi$ and $\operatorname{dim} \varphi=\aleph_{0}<c$. Thus Corollary 8.2 implies that there are no supercyclic strongly continuous operator semigroups $\left\{T_{t}\right\}_{t \in \mathbb{R}_{+}}$on $\omega$.

The rest of the section is devoted to the proof of Theorem 8.1. We need some preparation.

Lemma 8.3 Let $X$ be a finite dimensional topological vector space of the real dimension $>2$. Then there is no supercyclic strongly continuous operator semigroup $\left\{T_{t}\right\}_{t \in \mathbb{R}_{+}}$on $X$.

Proof. As well-known, any strongly continuous operator semigroup $\left\{T_{t}\right\}_{t \in \mathbb{R}_{+}}$on $\mathbb{K}^{n}$ has shape $\left\{e^{t A}\right\}_{t \in \mathbb{R}}$, where $A \in L\left(\mathbb{K}^{n}\right)$. Assume the contrary. Then there exists $n \in \mathbb{N}$ and $A \in L\left(\mathbb{K}^{n}\right)$ such that the semigroup $\left\{e^{t A}\right\}_{t \in \mathbb{R}}$ is supercyclic and $n \geq 3$ if $\mathbb{K}=\mathbb{R}, n \geq 2$ if $\mathbb{K}=\mathbb{C}$. Since the operators $e^{t A}$ are invertible and commute with each other, Proposition $\mathrm{G}$ implies that the set $W$ of universal elements for the family $\left\{z e^{t A}: z \in \mathbb{K}, t \in \mathbb{R}_{+}\right\}$is dense in $\mathbb{K}^{n}$. On the other hand, for each $\mathrm{c}>0$ and any $x \in \mathbb{K}^{n}$, from the restrictions on $n$ it follows that the closed set $\left\{z e^{t A} x: z \in \mathbb{K}, 0 \leq t \leq c\right\}$ is nowhere dense in $\mathbb{K}^{n}$ (one can use smoothness of the map $(z, t) \mapsto z e^{t A} x$ to see that the topological dimension of $\left\{z e^{t A} x: z \in \mathbb{K}, 0 \leq t \leq c\right\}$ is less than that of $\left.\mathbb{K}^{n}\right)$. Hence, each $x \in W$ is universal for the family $\left\{z e^{t A}: z \in \mathbb{K}, t>c\right\}$ for any $c>0$. Now if $(\mathrm{a}, \mathrm{b})$ is a finite subinterval of $(0, \infty)$, it is easy to see that the family $\left\{z e^{t k A}: z \in \mathbb{K}, a<t<b, k \in \mathbb{Z}_{+}\right\}$contains $\left\{z e^{t A}: z \in \mathbb{K}, t>c\right\}$ for a sufficiently large $c>0$. It follows that for each $x \in W$ the set $\left\{z e^{t k A} x: z \in \mathbb{K}, a<t<b, k \in \mathbb{Z}_{+}\right\}$is dense in $\mathbb{K}^{n}$. Taking into account that $(a, b)$ is arbitrary and $W$ is dense in $\mathbb{K}^{n}$, we see that $\left\{\left(t, x, z e^{t k A} x: t \in \mathbb{R}_{+}, z \in \mathbb{K}, x \in \mathbb{R}^{n}, k \in \mathbb{Z}_{+}\right\}\right.$is dense in $\mathbb{R}_{+} \times \mathbb{K}^{n} \times \mathbb{K}^{n}$. Applying Theorem 6.3, we see that for a generic $t \in$ $\mathbb{R}_{+}$in the Baire category sense the operator $e^{t A}$ is supercyclic. This contradicts the well-known fact that there are no supercyclic operators on finite dimensional spaces of real dimension $>2$.

The following lemma appears as Lemma 2 in reference [38]. It is worth noting that under the Continuum Hypothesis its statement becomes trivial.
Lemma 8.4 Let $(M, d)$ be a separable complete metric space and $X$ be a topological vector space. Then for any continuous map $f: M \rightarrow X$, the algebraic dimension of spanf $(M)$ is either finite or countable or continuum.

We use the above lemma to prove the following dichotomy.

Lemma 8.5 Let $\left\{T_{t}\right\}_{t \in \mathbb{R}_{+}}$be a strongly continuous operator semigroup on a topological vector space $X$ and $x \in X$. Then the space $C(x)=\operatorname{span}\left\{T_{t} x: t \in \mathbb{R}_{+}\right\}$is either finite dimensional or has dimension $c$.

Proof. From Lemma 8.4 it follows that $\operatorname{dim} C(x)$ is either finite or $\aleph_{0}$ or $c$. Thus it suffices to rule out the case $\operatorname{dim} C(x)=\aleph_{0}$.

Assume that $\operatorname{dim} C(x)=\aleph_{0}$. Restricting the operators $T_{t}$ to the invariant subspace $C(x)$, we can without loss of generality assume that $C(x)=X$. Thus $\operatorname{dim} X=\aleph_{0}$ and therefore $X$ is the union of an increasing sequence $\left\{E_{n}\right\}_{n \in \mathbb{Z}_{+}}$of finite dimensional subspaces. For each $\varepsilon>0$ let $X_{\varepsilon}=\operatorname{span}\left\{T_{t} x: t \geq \varepsilon\right\}$. First, we shall show that each $X_{\varepsilon}$ is finite dimensional. Let $\varepsilon>0,0<\alpha<\varepsilon$ and $A_{n}=\left\{t \in[\alpha, \varepsilon]: T_{t} x \in E_{n}\right\}$ for $n \in \mathbb{Z}_{+}$. Clearly $A_{n}$ are closed subsets of the interval $[\alpha, \varepsilon]$ and $[\alpha, \varepsilon]=\bigcup_{n=0}^{\infty} A_{n}$ since $X$ is the union of $E_{n}$. By the Baire category theorem there is $n \in \mathbb{Z}_{+}$such that $A_{n}$ has non-empty interior in $[\alpha, \varepsilon]$. Hence we can pick $a, b \in \mathbb{R}$ such that $\alpha \leq a<b \leq \varepsilon$ and $T_{t} x \in E_{n}$ for any $t \in[a, b]$. We shall show that $X_{a} \subseteq E_{n}$. Assume, it is not the case. Then the number $c=\inf \left\{t \in[a, \infty): T_{t} x \notin E_{n}\right\}$ belongs to $[b, \infty)$. Since the set $\left\{t \in \mathbb{R}_{+}: T_{t} \in E_{n}\right\}$ is closed, $T_{c} x \in E_{n}$. Since $[a, b]$ is uncountable and the span of $\left\{T_{t}: t \in[a, b]\right\}$ is finite dimensional, we can pick $a \leq t_{0}<t_{1}<\ldots<t_{n} \leq b$ and $c_{1}, \ldots, c_{n-1} \in \mathbb{K}$ such that

$$
T_{t_{n}} x=\sum_{j=1}^{n-1} c_{j} T_{t_{j}} x
$$

Since $T_{c} x \in E_{n}$, by definition of $c$, we can pick $t \in\left(c, c+t_{n}-t_{n-1}\right)$ such that $T_{t} x \notin E_{n}$. Since $t>c \geq t_{n}$, formula (8.1) implies that

$$
T_{t} x=T_{t-t_{n}} T_{t_{n}} x=T_{t-t_{n}} \sum_{j=1}^{n-1} c_{j} T_{t_{j}} x=\sum_{j=1}^{n-1} c_{j} T_{t-t_{n}+t_{j}} x \in E_{n} .
$$

because $a \leq t-t_{n}+t_{j} \leq c$ for $1 \leq j \leq n-1$. This contradiction proves that $X_{\varepsilon} \subseteq X_{a} \subseteq E_{n}$. Thus $X_{\varepsilon}$ is finite dimensional for each $\varepsilon>0$. Since $T_{t}(X)=T_{t}(C(x)) \subseteq X_{t}$, it follows that $T_{t}$ has finite rank for any $t>0$.

Now assume that $t>0$. Since $T_{t}$ has finite rank, $F_{t}=\operatorname{ker} T_{t}$ is a closed subspace of $X$ of finite codimension. It is also clear that $F_{t}$ is $T_{s}$-invariant for each $s \in \mathbb{R}_{+}$. Passing to quotient operators, $S_{s} \in L\left(X / F_{t}\right), S_{s}\left(u+F_{t}\right)=T_{s} u+F_{t}$, we arrive to a strongly continuous semigroup $\left\{S_{s}\right\}_{s \in \mathbb{R}_{+}}$on the finite dimensional space $X / F_{t}$. Hence there is $A \in L\left(X / F_{t}\right)$ such that $S_{s}=e^{s A}$ for any $s \in \mathbb{R}_{+}$. Thus each $S_{s}$ is invertible. Since each $S_{s}$ is a quotient of $T_{s}$, we see that

$$
r k T_{s} \geq r k S_{s}=\operatorname{dim} X / F_{t}=r k T_{t} \text { for any } \mathrm{t}>0 \text { and } \mathrm{s} \geq 0 .
$$

It follows that $T_{t}$ for $t>0$ have the same rank $k \in \mathbb{N}$. Passing to the limit as $t \rightarrow 0$, we see that the identity operator $I=T_{0}$ is the strong operator topology limit of a sequence of rank $k$ operators. Hence $r k I \leq k$. That is, $X$ is finite dimensional. This contradiction completes the proof. 
Lemma 8.6 Let $X$ be a topological vector space in which the linear span of each metrizable compact subset has dimension $<c$. Then for any strongly continuous operator semigroup $\left\{T_{t}\right\}_{t \in \mathbb{R}_{+}}$on $X$ and any $x \in X$, the space $C(x)=\operatorname{span}\left\{T_{t} x: t \in \mathbb{R}_{+}\right\}$is finite dimensional.

Proof. Let $\left\{T_{t}\right\}_{t \in \mathbb{R}_{+}}$be a strongly continuous operator semigroup on $X$ and $x \in X$. Strong continuity of $\left\{T_{t}\right\}_{t \in \mathbb{R}_{+}}$implies that for any $n$ $\in \mathbb{N}$, the set $K_{s}=\left\{T_{t} x: 0 \leq t \leq n\right\}$ is compact and metrizable. Hence $\operatorname{dim} E_{n}<\boldsymbol{c}$ for any $n \in \mathbb{N}$, where $E_{n}=\operatorname{span}\left(K_{n}\right)$. Since the sum of countably many cardinals strictly less than $\mathfrak{c}$ is strictly less than $\mathfrak{c}$, we see that

$$
\operatorname{dim} C(x)=\operatorname{dim}\left(\bigcup_{n=1}^{\infty} E_{n}\right) \leq \sum_{n=1}^{\infty} \operatorname{dim} E_{n}<\boldsymbol{c} .
$$

By Lemma $8.5, C(x)$ is finite dimensional.

Proposition 8.7 Let $X$ be an infinite dimensional locally convex space such that in $X_{\sigma}^{\prime}$ the span of any compact metrizable subset has dimension $<c$. Then there is no strongly continuous supercyclic operator semigroup $\left\{T_{t}\right\}_{t \in \mathbb{R}_{+}}$on $X$.

Proof. Assume that there exists a supercyclic strongly continuous operator semigroup $\left\{T_{t}\right\}_{t \in \mathbb{R}_{+}}$on $X$. It is straightforward to verify that $\left\{T_{t}^{\prime}\right\}_{t \in \mathbb{R}_{+}}$is a strongly continuous semigroup on $X_{\sigma}^{\prime}$. Pick three linearly independent vectors $f_{1}, f_{2}$ and $f_{3}$ in $X^{\prime}$. By Lemma 8.6, $E_{j}=\operatorname{span}\left\{T_{t}^{\prime} f_{j}: t \in \mathbb{R}_{+}\right\}$is finite dimensional for $1 \leq j \leq 3$. Clearly each $E_{\mathrm{j}}$ is $T_{t}^{\prime}$-invariant for any $t \in \mathbb{R}_{+}$. Then $E=E_{1}+E_{2}+E_{3}$ is finite dimensional and $T_{t}^{\prime}$-invariant for any $t \in \mathbb{R}_{+}$. Since $f_{j} \in E$ for $1 \leq j \leq 3, \operatorname{dim} E \geq 3$. Since $E$ is $T_{t}^{\prime}$ invariant, we see that its annihilator $F=\{x \in X: f(x)=0$ forany $f \in E\}$ if $T_{t}$-invariant for each $t \in \mathbb{R}_{+}$. Thus we can consider the quotient operators $S_{t} \in L(X / F), S_{t}(x+F)=T_{t} x+F$. Clearly $\left\{S_{t}\right\}_{t \in \mathbb{R}_{+}}$is a strongly continuous operator semigroup on $X / F$. Moreover, $\left\{S_{t}\right\}_{t \in \mathbb{R}_{+}}$is supercyclic since $\left\{T_{t}\right\}_{t \in \mathbb{R}_{+}}$is. Now since $\operatorname{dim} E=\operatorname{dim} X / F, X / F$ is finite dimensional and has dimension $\geq 3$. By Lemma 8.3 , there are no strongly continuous supercyclic operator semigroups on $X / F$. This contradiction completes the proof.

Proof of Theorem 8.1. If $X$ has no compact metrizable subsets whose linear span has dimension $\mathfrak{c}$, Lemma 8.6 implies that the linear span of any orbit $\left\{T_{t} x: t \in \mathbb{R}_{+}\right\}$is finite dimensional. It follows that $\left\{T_{t}\right\}_{t \in \mathbb{R}_{+}}$is not supercyclic. It remains to consider the case when $X_{\sigma}^{\prime}$ has no compact metrizable subsets whose linear span has dimension $\mathbf{c}$ and apply Proposition 8.7.

\section{The space $\varphi$}

Recall that $\varphi$ is a linear space of countable algebraic dimension carrying the strongest locally convex topology. In this section we mention certain properties of $\varphi$, mainly those which are related to continuous linear operators. It is well known [17] that $\varphi$ is complete and all linear subspaces of $\varphi$ are closed. Moreover, infinite dimensional subspaces of $\varphi$ are isomorphic to $\varphi$. It is also well-known that for any topology $\theta$ on $\varphi$ such that $(\varphi, \theta)$ is a topological vector space, $\theta$ is weaker than the original topology of $\varphi$. The latter observation immediately implies the following lemma.

Lemma 9.1 For any topological vector space $X$ and any linear map $T: \varphi \rightarrow X, T$ is continuous.
Lemma 9.2 Let $X$ be a topological vector space and $T: X \rightarrow \varphi$ be a surjective continuous linear operator. Then $X$ is isomorphic to $\varphi \times k e r T$.

Proof. Since $T$ is linear and surjective, there exists a linear map $S: \varphi \rightarrow X$ such that $T S=I$. By Lemma 9.1, $S$ is continuous. Consider the linear maps

$A: \varphi \times \operatorname{ker} T \rightarrow X, \quad A(u, y)=y+S u$ and $B: X \rightarrow \varphi \times \operatorname{ker} T, \quad B x=(T x, x-S T x)$.

It is easy to see that $A$ and $B$ are continuous and that $A B=I$ and $B A=I$. Hence $B$ is a required isomorphism.

Corollary 9.3 Let $X$ be a topological vector space. Then the following conditions are equivalent. $p t$

(9.3.1) $X$ is isomorphic to a space of the shape $Y \times \varphi$, where $Y$ is a topological vector space'

(9.3.2) $X$ has a quotient isomorphic to $\varphi$;

(9.3.3) there is $T \in L(X, \varphi)$ such that $T(X)$ is infinite dimensional.

Proof. The implications $(9.3 .1) \Rightarrow(9.3 .2) \Rightarrow(9.3 .3)$ are trivial. Assume that (9.3.3) is satisfied. That is, there is $T \in L(X, \varphi)$ with infinite dimensional $T(X)$. Since any infinite dimensional linear subspace of $\varphi$ is isomorphic to $\varphi$, we see that $T(X)$ is isomorphic to $\varphi$. Hence there is a surjective $S \in L(X, \varphi)$. By Lemma $9.2 X$ is isomorphic to $Y \times \varphi$, where $Y=\operatorname{ker} S$. Hence (9.3.3) implies (9.3.1), which completes the proof.

\section{Cyclic operators on $\varphi$}

Clearly $\varphi$ is isomorphic to the space $\mathcal{P}$ of all polynomials over $\mathbb{K}$ endowed with the strongest locally convex topology. The shift operator on $\varphi$ is obviously similar to the operator

$M: \mathcal{P} \rightarrow \mathcal{P}, \quad M p(z)=z p(z)$.

For each $n \in \mathbb{N}$ we denote

$\mathcal{P}_{n}=\{p \in \mathcal{P}: \operatorname{deg} p<n\}$.

Clearly $\mathcal{P}_{n}$ is an $n$-dimensional subspace of $\mathcal{P}$.

Lemma 9.4 An operator $T \in L(\varphi)$ is cyclic if and only if $T$ is similar to the operator $M$.

Proof. Clearly 1 is a cyclic vector for $M$. Hence any operator similar to $M$ is cyclic. Now let $T \in L(\varphi)$ be cyclic and $x \in \varphi$ be a cyclic vector for $T$. Then the vectors $T^{n} x$ for $n \in \mathbb{Z}$ are linearly independent. Indeed, otherwise their span is finite dimensional, which contradicts cyclicity of $x$ for $T$. Since any linear subspace of $\varphi$ is closed, we see that $\left\{T^{n} x: n\right.$ $\left.\in \mathbb{Z}_{+}\right\}$is an algebraic basis of $\varphi$. It is easy to see then that the linear map $J: \mathcal{P} \rightarrow \varphi, J p=p(T) x$ is invertible and $T=J M J^{-1}$. By Lemma 9.1, $J$ and $J^{-1}$ are continuous. Hence $T$ is similar to $M$.

We need a multicyclic version of the above lemma.

Lemma 9.5 Let $T \in L(\varphi)$. Then the following conditions are equivalent.

(9.5.1) $T$ is multicyclic;

(9.5.1) there exists $k \in \mathbb{Z}$ and a linear subspace $Y$ of $\varphi$ of finite codimension such that $T(Y) \subseteq Y$ and the restriction $\left.T\right|_{Y} \in L(Y)$ is similar to $M^{k}$, where $M$ is the operator defined in (9.1).

Proof. First, assume that (9.5.2) is satisfied. Pick a finite dimensional subspace $Z$ of such that $\varphi=Z \oplus Y$. Since $\left.T\right|_{Y}$ is similar to $M^{k}$, we can pick an invertible linear operator $J: \mathcal{P} \rightarrow \varphi$ such that $T y=J M^{k} J^{-1} y$ for any $y \in Y$. Let $L=Z+J\left(\mathcal{P}_{k}\right)$, where $\mathcal{P}_{k}$ is defined in (9.8). Clearly 
$L$ is a finite dimensional subspace of $\varphi$. From the equality $\left.T\right|_{Y}=J M^{k} J^{-1}$ it easily follows that for any $n \in \mathbb{N}$,

$$
L+T(L)+\ldots+T^{n-1}(L) \supseteq Z+J\left(\mathcal{P}_{n k}\right) .
$$

Hence the linear span of the union of $T^{j}(L)$ for $j \in \mathbb{Z}_{+}$contains $Z+J(\mathcal{P})=Z+Y=\varphi$. Thus $T$ is $m$-cyclic with $m=\operatorname{dim} L$. The implication (9.5.2) $\Rightarrow(9.5 .1)$ has been verified.

Assume now that $T$ is $n$-cyclic for some $n \in \mathbb{N}$. Then there is an $n$ -dimensional subspace $L$ of $\varphi$ such that

$$
\operatorname{span}\left(\bigcup_{k=0}^{\infty} T^{n}(L)\right)=\varphi
$$

(again, we use the fact that any linear subspace of $\varphi$ is closed and therefore a dense subspace of $\varphi$ must coincide with $\varphi$ ). We will use the concept of $T$-independence, introduced in Section 7. Since $T$ -independence implies linear independence, any $T$-independent subset of $L$ has at most $n$ elements. Let $k$ be the maximum of cardinalities of $T$-independent subsets of $L$ and $A$ be a $T$-independent subset of cardinality $k$. Since $A$ is linearly independent, we can pick a subset $B \subset L$ of cardinality $n-k$ such that $A \cup B$ is a basis in $L$. From the definition of $k$ it follows that for any $b \in B, A \cup\{b\}$ is not $T$ independent and therefore using $T$-independence of $A$, we can find polynomials $p_{b}$ and $p_{b, a}$ for $a \in A$ such that

$$
p_{b} \neq 0 \text { and } p_{b}(T) b=\sum_{a \in A} p_{b, a}(T) a .
$$

Now let $m=0$ if $B=\varnothing$ and $m=\max _{b \in B} \operatorname{deg} p_{b}$ otherwise. Consider the spaces

$$
Z=\operatorname{span}\left\{T^{j} b: b \in B, 0 \leq j \leq m\right\} \text { and } Y=\operatorname{span}\left\{T^{j} a: a \in A, j \in \mathbb{Z}_{+}\right\} .
$$

Then $Z$ is finite dimensional and $T(Y) \subseteq Y$. Obviously,

$$
T^{j} a \in Y \subseteq Y+Z \quad \text { foranya } \in \operatorname{Aandj} \in \mathbb{Z}_{+} .
$$

Let $b \in B$ and $j \in \mathbb{Z}_{+}$. Since $p_{b} \neq 0$ and $\operatorname{deg} p_{b} \leq m$, we can find polynomials $q, r$ such that $\operatorname{deg} r<m$ and $t^{j}=q(t) p_{b}(t)+r(t)$. Then $T^{j} b=q(T) p_{b}(T) b+r(T) b$. Since $\operatorname{deg} r<m, r(T) b \in Z$. According to (9.4), $p_{b}(T) b \in Y$. Since $Y$ is invariant for $T, q(T) p_{b}(T) b \in Y$. Thus

$$
T^{j} b \in Y+Z \quad \text { for any } \mathrm{b} \in \mathrm{B} \text { and } \mathrm{j} \in \mathbb{Z}_{+} .
$$

Since $A \cup B$ is a basis of $L$, from (9.5) and (9.6) it follows that $T^{j}(L) \subseteq Y+Z$ for each $j \in \mathbb{Z}_{+}$. According to (9.3), $\phi=Y+Z$. Since $Z$ is finite dimensional, we see that $Y$ has finite codimension in $\varphi$. In particular, $Y$ is non-trivial and therefore $A \neq \varnothing$. That is, $1 \leq k \leq n$ and $A=\left\{a_{1}, \ldots, a_{k}\right\}$. Now consider the linear operator $J: \mathcal{P} \rightarrow Y$, which sends the monomial $t^{l}$ to $T^{j} a_{s}$, where $j \in \mathbb{Z}_{+}$and $s \in\{1, \ldots, k\}$ are uniquely defined by the equation $l+1=j k+s$. By definition of $Y, J$ is onto. From $T$-independence of $A$ it follows that $J$ is also one-to-one. By definition of $J$, we have $J t^{l+k}=T J t^{l}$. Hence $J M^{k} p=T J p$ for any $p \in \mathcal{P}$. That is, $M^{k}$ and $\left.T\right|_{Y}$ are similar.

Corollary 9.6 Let T be a multicyclic operator on $\varphi$. Then $T$ is not onto.

Proof. According to Lemma 9.5, we can decompose $\varphi$ into a direct sum $\varphi=Y \oplus Z$, where $Z$ has finite dimension $m \in \mathbb{Z}_{+}, T(Y) \subseteq Y$ and
$\left.T\right|_{Y}$ is similar to $M^{k}$ for some $k \in \mathbb{N}$. Since $\left.T\right|_{Y}$ is similar to $M^{k}, T^{m+1}(Y)$ has codimension $k(m+1)>m$ in $Y$. Hence $\operatorname{dim} \varphi / T^{m+1}(Y)>m$. On the other hand, $\operatorname{dim} T^{m+1}(Z) \leqslant \operatorname{dim} Z=m$. Thus $T^{m+1}(\varphi)=T^{m+1}(Z)+T^{m+1}(Y)$ has positive codimension in $\varphi$. Hence $T^{m+1}$ is not onto and so is $T$.

\section{Proof of Theorem 1.12}

In this section $X$ is a topological vector space, which has no quotient isomorphic to $\varphi$. We have to show that there are no cyclic operators with dense range on $X \times \varphi$. Assume the contrary and let $T \in L(X \times \varphi)$ be a cyclic operator with dense range. Consider the matrix representation of $T$ :

$T=\left(\begin{array}{ll}A & B \\ C & D\end{array}\right)$, where $A \in L(X), B \in L(\varphi, X), C \in L(X, \varphi)$ and $D \in L(\varphi)$

With $T$ acting according to the formula $T(x, u)=(A x+B u, C x+D u)$. Since $T$ is cyclic, we can pick a vector $(x, u) \in X \times \varphi$ such that $E=\operatorname{span}\left\{T^{k}(x, u): k \in \mathbb{Z}_{+}\right\} \quad$ is dense in $X \times \varphi$. Since $T$ has dense range, then $T^{m}$ has dense range for any $m \in \mathbb{Z}_{+}$. Thus $E_{m}=T^{m}(E)=\operatorname{span}\left\{T^{k}(x, u): k \geq m\right\}$ is dense in $X \times \varphi$ for each $m$ $\in \mathbb{Z}_{+}$. Let $T^{k}(x, u)=\left(x_{k}, u_{k}\right)$ for $k \in \mathbb{Z}_{+}$, where $x_{k} \in X$ and $u_{k}=\varphi$. Since $E_{m}=\operatorname{span}\left\{\left(x_{k}, u_{k}\right): k \geq m\right\}$ is dense in $X \times \varphi$, we see that $F_{m}=\operatorname{span}\left\{u_{k}: k \geq m\right\}$ is dense in $\varphi$ for any $m \in \mathbb{Z}_{+}$. Hence $F_{m}=\varphi$ for any $m \in \mathbb{Z}_{+}$. Since $X$ has no quotients isomorphic to $\varphi$, Lemma 9.3 implies that $L_{0}=C(X)$ is a finite dimensional subspace of $\varphi$. Then the space

$$
L=\operatorname{span}\left(L_{0} \cup\{u\}\right) .
$$

is also finite dimensional. Clearly $u_{0}=u \in L$ and $u_{k+1}=C x_{k}+D u_{k} \in D u_{k}+L$ for any $k \in \mathbb{Z}_{+}$. It follows that each $u_{k}$ belongs to the space spanned by the union of $D^{m}(L)$ for $m \in$ $\mathbb{Z}_{+}$. Since $L$ is finite dimensional, $D$ is multicyclic. By Lemma 9.5, we can decompose $\varphi$ into a direct sum $\varphi=Y \oplus Z$, where $Z$ is finite dimensional, $D(Y) \subseteq Y$ and $\left.D\right|_{Y}$ is similar to $M^{n}$ for some $n \in \mathbb{N}$. That is, there exists an invertible $J \in L(Y, \mathcal{P})$ such that $\left.D\right|_{Y}=J^{-1} M^{n} J$. Let also $P \in L(\varphi)$ be the linear projection onto $Y$ along $Z$. We consider two cases.

Case 1. The sequence $\left\{\operatorname{deg} J P u_{k}\right\}$ is bounded from above. In this case $\operatorname{span}\left\{J P u_{k}: k \in \mathbb{Z}_{+}\right\}$is finite dimensional. Since $J$ is invertible, $\operatorname{span}\left\{P u_{k}: k \in \mathbb{Z}_{+}\right\}$is finite dimensional. Since $P$ has finite dimensional kernel, $F_{0}=\operatorname{span}\left\{u_{k}: k \in \mathbb{Z}_{+}\right\}$is finite dimensional. We have arrived to a contradiction with the equality $F_{0}=\varphi$.

Case 2. The sequence $\left\{d e g J P u_{k}\right\}$ is unbounded from above. Since $N=(L+Z+D(Z)) \cap Y$ is finite dimensional,

$$
m=\sup _{w \in N \backslash\{0\}} \operatorname{deg} J w \in \mathbb{Z}_{+} .
$$

We shall show that $\operatorname{deg} J P u_{k+1}=n+\operatorname{deg} J P u_{k} \quad$ whenever $\operatorname{deg} J P u_{k}>m$. Indeed, let $k \in \mathbb{Z}_{+}$be such that $\operatorname{deg} J P u_{k}>m$. By definition of $P, u_{k}-P u_{k} \in Z$ and $u_{k+1}-P u_{k+1} \in Z$. As we know, $u_{k+1} \in D u_{k}+L$. Hence $P u_{k+1} \in D P u_{k}+L+Z+D(Z)$. Since $P u_{k+1}$ and $D P u_{k}$ belong to, $Y$ we have $P u_{k+1} \in D P u_{k}+N$. Thus there is $w \in N$ such that $P u_{k+1}=D P u_{k}+w$. Hence $J P u_{k+1}=J D P u_{k}+J w=M^{n} J P u_{k}+J w$. 
Since $\quad \operatorname{deg} M^{n} J P u_{k}=n+\operatorname{deg} J P u_{k}>m \geqslant \operatorname{deg} J w, \quad$ we have $\operatorname{deg} J P u_{k+1}=n+\operatorname{deg} J P u_{k}$ (the degree of the sum of two polynomials of different degrees equals to the maximum of the degrees). Since the sequence $\left\{\operatorname{deg} J P u_{k}\right\}$ is unbounded from above, there is $k \in \mathbb{Z}_{+}$such that $\operatorname{deg} J P u_{k}>m$ and according to the just proven statement, we will have $\operatorname{deg} J P u_{j}=\operatorname{deg} J P u_{k}+n(j-k)$ for $j \geq k$. Since any family of polynomials with pairwise different degrees is linearly independent, we see that the vectors $J P u_{j}$ for $j \geq k$ are linearly independent. Since $J P$ is a linear operator, the vectors $u_{j}$ for $j \geq k$ are linearly independent in $\varphi$. Hence the sequence of spaces $F_{j}$ for $j \geq k$ is strictly decreasing. On the other hand, we know that $F_{j}=\varphi$ for each $j \in \mathbb{Z}_{+}$. This contradiction completes the proof.

\section{Hypercyclicity of operators on direct sums}

We shall prove the following lemma, which is a key for the proof of Theorem 1.13.

Lemma 10.1 Let $\left\{X_{n}\right\}_{n \in \mathbb{Z}_{+}}$be a sequence of infinite dimensional locally convex spaces such that

(10.1.1) there exists a dense linear subspace $Y$ of $X_{0}$, carrying a topology, stronger than the one inherited from $X_{0}$ and turning $Y$ into a separable metrizable topological vector space;

(10.1.2) there exists $T_{0} \in L\left(X_{0}, X_{0} \oplus X_{1}\right)$ with dense range;

(10.1.3) for each $n \in \mathbb{N}$, there exists $T_{n} \in L\left(X_{n}, X_{n+1} \times \mathbb{K}\right)$ with dense range.

Then there is a hypercyclic operator $S$ on $X=\bigoplus_{n=0}^{\infty} X_{n}$.

It is worth noting that condition (10.1.1) implies that $X_{0}$ is separable, condition (10.1.2) implies that $X_{1}$ is separable and condition (10.1.3) implies that $X_{n}$ for $n \geq 2$ are all separable. Thus $X$ is separable. We need the following auxiliary lemma.

Lemma 10.2 Let $X$ and $Y$ be topological vector spaces such that there exists $T \in L(X, Y \times \mathbb{K})$ with dense range. Then for any closed hyperplane $H$ of $X$, there exists $S \in L(H, Y)$ with dense range.

Proof. Let $T_{0} \in L(H, Y \times \mathbb{K})$ be the restriction of $T$ to $H$. We can write $T_{0}=\left(S_{0}, g\right)$, where $S_{0} \in L(H, Y)$ and $g \in H^{\prime}$. If $T_{0}$ has dense range, then $S=S_{0}$ is a continuous linear operator from $H$ to $Y$ with dense range. It remains to consider the case when the range of $T_{0}$ is not dense. Since the range of $T$ is dense and $T_{0}$ is a restriction of $T$ to a closed hyperplane, the codimension of the closure of $T_{0}(H)$ in $Y \times \mathbb{K}$ does not exceed 1. Thus the codimension of the closure of $T_{0}(H)$ in $Y$ $\times \mathbb{K}$ is exactly 1 and there is a non-zero $\psi \in(Y \times \mathbb{K})^{\prime}$ such that $T_{0}(H)$ is a dense subspace of ker $\psi$. If $k e r \psi=Y \times\{0\}$, then again we can take $S=S_{0}$. If $k e r \psi \neq Y \times\{0\}$, we can pick $y \in Y$ such that $\psi(y)=1$. Take $S \in L(H, Y), S x=S_{0} x+g(x) y$. It is straightforward to verify that $S$ has dense range. and

Proof of Lemma 10.1. Let $\left\{U_{n}\right\}_{n \in \mathbb{Z}_{+}}$be a base of topology of $Y$

$$
Z_{n}=\left\{x \in X: x_{j}=0 \text { for } j>n\right\} .
$$

Clearly $X$ is the union of the increasing sequence of subspaces $Z_{n}$ and each $Z_{n}$ is naturally isomorphic to $\bigoplus_{k=0}^{n} X_{k}$. We shall construct inductively a sequence of operators $S_{k} \in L\left(Z_{k}, Z_{k+1}\right)$ and vectors $y_{k} \in Y$ satisfying the following conditions for any $k \in \mathbb{Z}$ :

(a1) $S_{j} x=S_{k} x$ for any $j<k$ and $x \in Z_{j}$;

(a2) $S_{k}\left(Z_{k}\right)$ is dense in $Z_{k+1}$;

(a3) $S_{k} \ldots S_{0} y_{k} \notin Z_{k}$;

(a4) $S_{k} \ldots S_{0} y_{k-1}=y_{k}$ if $k \geq 1$;

(a5) $y_{k} \in U_{k}$.

By (10.1.2) there exists $S_{0} \in L\left(Z_{0}, Z_{1}\right)$ with dense range. Since $Y$ is dense in $X_{0=} Z_{0}, S_{0}$ has dense range and $Z_{0}$ is nowhere dense in $Z_{1}$, we can pick $y_{0} \in U_{0}$ such that $S_{0} y_{0} \notin Z_{0}$. The basis of induction has been constructed. Assume now that $n \in \mathbb{N}$ and $y_{k} \in Y, S_{k} \in L\left(Z_{k}, Z_{k+1}\right)$ satisfying (a1-a5) for $k<n$ are already constructed. According to (a3) for $k=n-1$, we have $w=S_{n-1} \ldots S_{0} y_{n-1} \notin Z_{n-1}$. That is, the $n^{\text {th }}$ component $w_{n}$ of $w$ is non-zero. Since $X_{n}$ is locally convex, we can pick a closed hyperplane $H$ in $X_{n}$ such that $w_{n} \notin H$. Let $P$ be the linear projection on $Z_{n}$ onto $H$ along $Z_{n-1} \oplus \operatorname{span}\left\{w_{n}\right\}$. From (10.1.3) and Lemma 10.2 it follows that there is $R \in L\left(H, X_{n+1}\right)$ with dense range. According to (a3) for $k=n-1$, the operator $S_{n-1} \ldots S_{0}$ from $\mathrm{Z}_{0}$ to $\mathrm{Z}_{n}$ has dense range. Hence the operator $Q=R P S_{n-1} \ldots S_{0}$ from $Z_{0}$ to $X_{n+1}$ has dense range. Since $Y$ is dense in $Z_{0}$, we can pick $y_{n} \in U_{n}$ such that $Q y_{n} \neq 0$. Now we define the operator $S_{n}: Z_{n} \rightarrow Z_{n+1}$. It is easy to see that $Z_{n}=Z_{n-1} \oplus H \oplus \operatorname{span}\{w\}$. We set

$$
S_{n}(x+y+s w)=S_{n-1} x+R y+s y_{n} \text { for } x \in Z_{n-1}, y \in H \text { and } s \in \mathbb{K} \text {. }
$$

The operator $S_{n}$ is continuous since $S_{n-1}$ and $R$ are continuous. Clearly (a1) and (a5) for $k=n$ are satisfied. Next, $S_{n}\left(Z_{n}\right) \supseteq S_{n-1}\left(Z_{n-1}\right)+R(H)$. Since $S_{n-1}\left(Z_{n-1}\right)$ is dense in $Z_{n}$ (condition (a2) for $k=n-1$ ) and $R(H)$ is dense in $X_{n+1}$, we see that $S_{n}\left(Z_{n}\right)$ is dense in $Z_{n+1}=Z_{n} \oplus X_{n}$, which gives us (a2) for $k=n$. From the last display and the relation $Q y_{n} \neq 0$ it follows that (a3) is satisfied for $k=n$. Finally, since $S_{n} w=y_{n}$ from the definition of $w$ we see that (a4) for $k=n$ is also satisfied. Thus the inductive construction of $S_{k}$ and $Y_{k}$ is complete.

Condition (a2) ensures that there is a unique operator $S \in L(X)$ such that $\left.S\right|_{Z_{n}}=S_{n}$ for any $n \in \mathbb{Z}_{+}$. From (a4) it now follows that

$S^{k+1} y_{k-1}=y_{k}$ for each $\mathrm{k} \in \mathbb{Z}_{+}$.

According to the above display, the set $A=\left\{y_{n}: n \in \mathbb{Z}_{+}\right\}$is contained in the orbit $O\left(S, y_{0}\right)$. By (a5) $A$ is dense in $Y$. Since $Y$ is dense in $X_{0}$ and carries a stronger topology, $A$ is dense in $X_{0}=Z_{0}$. By (a2) $S^{m}(A)$ is dense in $Z_{m}$ for each $m \in \mathbb{Z}_{+}$. Since $A \subset O\left(S, y_{0}\right)$, we have that $S^{m}(A) \subset O\left(S, y_{0}\right)$ and therefore $O\left(S, y_{0}\right) \cap Z_{m}$ is dense in $Z_{m}$ for each $m \in \mathbb{Z}_{+}$. Hence $O\left(S, y_{0}\right)$ is dense in $X$. That is, $Y$ is a hypercyclic vector for $S$.

Remark 10.3 The orbit of the hypercyclic vector constructed in the proof of Lemma 10.1 is not just dense. It is sequentially dense. The latter property is strictly stronger than density already for countable direct sums of separable infinite dimensional Banach spaces.

\section{Proof of Theorem 1.14}

Let $X_{n} \in \mathfrak{M}$ for each $n \in \mathbb{Z}_{+}$and $x=\bigoplus_{n=0}^{\infty} X_{n}$. We shall apply Lemma 10.1. Condition (10.1.1) is satisfied according Lemma 3.3. Indeed $X_{1}$ 
admits a Banach $s$-disk with dense span. By Lemma 3.8, spaces $X_{0} \times X_{1}$ and $X_{n} \times \mathbb{K}$ belong to $\mathfrak{M}$. From Lemma 3.6 it follows that conditions $(10.1 .2)$ and (10.1.3) are also satisfied. Thus by Lemma 10.1, there is hypercyclic $T \in L(X)$.

\section{Proof of Theorem 1.13}

As we have already mentioned, s Fréchet space $X$ belongs to $\mathfrak{M}$ if and only if $X$ is infinite dimensional, separable and non-isomorphic to $\omega$. Moreover, in any separable Fréchet space there is an $\ell_{1}$-sequence with dense span.

Lemma 10.4 Let $X$ and $Y$ be separable infinite dimensional Fréchet spaces. Then the following conditions are equivalent

(10.4.1) there is no $T \in L(X, Y)$ with dense range;

(10.4.2) $X$ is isomorphic to $\omega$ and $Y$ is not isomorphic to $\Omega$.

Proof. If both $X$ and $Y$ are isomorphic to $\omega$, then obviously there is a surjective $T \in L(X, Y)$. If $X$ is isomorphic to $\omega, Y$ is not and $T \in L(X, Y)$, then $Z=T(X)$ carries minimal locally convex topology [29] since $\omega$ does. It follows that $Z$ is either finite dimensional or isomorphic to $\underline{\omega}$ and therefore complete. Hence $Z$ is closed in $Y$. It follows that $Z=\bar{Z} \neq Y$ since $Y$ is neither finite dimensional nor isomorphic to. Thus there is not $T \in L(X, Y)$ with dense range. It remains to show that there is $T \in$ $L(X, Y)$ with dense range if $X$ is not isomorphic to $\omega$. In this case the topology of $X$ is not weak and it remains to apply Lemma 3.6.

Lemma 10.5 Let $X$ be the countable locally convex direct sum of a sequence of separable Fréchet spaces infinitely many of which are infinite dimensional. Then one of the following two possibilities occurs:

(10.5.1) $X$ is isomorphic to $Y \oplus Z$, where $Y$ is a separable infinite dimensional Fréchet space and $Z$ is the locally convex direct sum of an infinite countable number of copies of $\omega$;

(10.5.2) $X$ is isomorphic to $\bigoplus_{n=0}^{\infty} Y_{n}$, where each $Y_{n}$ is a separable infinite dimensional Fréchet space non-isomorphic to $\omega$.

Proof. Separating the finite dimensional spaces, spaces isomorphic to $\omega$ and infinite dimensional spaces non-isomorphic to $\omega$, we see that

$$
X=\bigoplus_{\alpha \in A} X_{\alpha} \oplus \bigoplus_{\beta \in B} X_{\beta} \oplus \bigoplus_{\gamma \in C} X_{\gamma},
$$

where the sets $A, B$ and $C$ are pairwise disjoint, $X$ is isomorphic to $\omega$ for each $\alpha \in A, X_{\beta}$ is a separable infinite dimensional Fréchet space nonisomorphic to $\omega$ for any $\beta \in B, X_{\gamma}$ is finite dimensional for each $\gamma \in C$, $A \cup B$ is infinite and countable and $C$ is either finite or countable.

If $B$ and $C$ are finite, then $A$ is infinite. Pick $\alpha_{0} \in A$. Then

$$
X=Y \oplus \bigoplus_{\alpha \in A \backslash\left\{\alpha_{0}\right\}} X_{\alpha} \text {, where } Y=X_{\alpha_{0}} \oplus \bigoplus_{\beta \in B} X_{\beta} \oplus \bigoplus_{\gamma \in C} X_{\gamma} .
$$

Clearly $Y$ is a separable infinite dimensional Fréchet space. Since each $X_{\alpha}$ is isomorphic to $\omega$, we fall into the case (10.5.1). If $B$ is finite and $C$ is infinite, then $A$ is infinite and both $A$ and $C$ can be enumerated by elements of $\mathbb{Z}_{+}: A=\left\{\alpha_{n}: n \in \mathbb{Z}_{+}\right\}, C=\left\{y_{n}: n \in \mathbb{Z}_{+}\right\}$. Then

$$
X=Y \oplus \bigoplus_{n \in \mathbb{Z}_{+}}\left(X_{\alpha_{n+1}} \oplus X_{\gamma_{n}}\right) \text { where } Y=X_{\alpha_{0}} \oplus \bigoplus_{\beta \in B} X_{\beta} .
$$

Again $Y$ is a separable infinite dimensional Fréchet space. Since each $X_{\alpha_{n+1}} \oplus X_{\gamma_{n}}$ is isomorphic to $\omega,(10.5 .1)$ is satisfied. If $B$ is infinite and $A \cup C$ is infinite, then we enumerate both $B$ and $A \cup C$ by the elements of $\mathbb{Z}_{+}: B=\left\{\beta_{n}: n \in \mathbb{Z}_{+}\right\}, A \cup C=\left\{\rho_{n}: n \in \mathbb{Z}_{+}\right\}$. We arrive to

$$
X=\bigoplus_{n \in \mathbb{Z}_{+}}\left(X_{\beta_{n}} \oplus X_{\rho_{n}}\right) .
$$

Since each $X_{\beta_{n}} \oplus X_{\rho_{n}}$ is a separable infinite dimensional Fréchet space non-isomorphic to $\omega,(10.5 .2)$ is satisfied. Finally, if $B$ is infinite and $A \cup C$ is finite, we fix $\beta_{0} \in B$ and write

$$
X=Z \oplus \bigoplus_{\beta \in B \backslash\left\{\beta_{0}\right\}} X_{\beta} \text {, where } Z=X_{\beta_{0}} \oplus \bigoplus_{\rho \in A \cup C} X_{\rho} .
$$

Again $Z$ and each $X_{\beta}$ are separable infinite dimensional Fréchet spaces non-isomorphic to $\omega$ and we fall into the case (10.5.2).

We are ready to prove Theorem 1.13. Let $X$ be a countable infinite direct sum of separable Fréchet spaces. If all the spaces in the sum, except for finitely many, are finite dimensional, then $X$ is isomorphic to $Y \times \varphi$, where $Y$ is a Fréchet space. According to Theorem 1.12, $X$ admits no cyclic operator with dense range. In particular, there is no supercyclic operator on $X$. If there are infinitely many infinite dimensional spaces in the sum defining $X$, then according to Lemma 10.5 , we see that $X$ is isomorphic to

$$
Y=\bigoplus_{n \in \mathbb{Z}_{+}} Y_{n}
$$

where $Y_{n}$ are all separable infinite dimensional Fréchet spaces and either all $Y_{n}$ are non-isomorphic to $\omega$ or all $Y_{n}$ for $n \geq 1$ are isomorphic to . In any case from Lemma 10.4 it follows that there exists $T_{0} \in L\left(Y_{0}, Y_{0} \oplus Y_{1}\right)$ with dense range and for each $n \in \mathbb{N}$, there exists $T_{n} \in L\left(Y_{n}, Y_{n+1} \times \mathbb{K}\right)$ with dense range. By Lemma 10.1, there is a hypercyclic operator on $X$. The proof of Theorem 1.13 is complete.

\section{Hypercyclic operators on countable unions of spaces}

The following lemma is a main tool in the proof of Theorem 1.11.

Lemma 11.1 Let a locally convex space be the union of an increasing sequence $\left\{X_{n}\right\}_{n \in \mathbb{N}}$ of its closed linear subspaces. Assume also that for any $n \in \mathbb{N}$ there is an $\ell_{1}$-sequence with dense span in $X_{n}$ and the topology of $X_{n} / X_{n-1}$ is not weak, where $X_{0}=\{0\}$. Then there exists a linear map $S: X \rightarrow X$ and $x_{0} \in X_{1}$ such that for any $n \in \mathbb{N},\left.S\right|_{X_{n}} \in L\left(X_{n}, X_{n+1}\right)$ and the orbit $O(S, x)=\left\{S^{k} x: k \in \mathbb{Z}_{+}\right\}$is dense in $X$.

Note that we do not claim continuity of the above operator $S$ on $X$. Although if, for instance, $X$ is the inductive limit of the sequence $\left\{X_{n}\right\}$, then continuity of $S$ will immediately follow from the continuity of the restrictions $\left.S\right|_{X_{n}}$.

Proof of Lemma 11.1. For each $n \in \mathbb{N}$, let $\left\{x_{n, k}\right\}_{k \in \mathbb{Z}_{+}}$be an $\ell_{1}$-sequence with dense span in $X_{n}$. For any $n \in \mathbb{N}$, we apply Lemma 3.1 with the triple of spaces $\left(Y, Y_{1}, Y_{0}\right)$ being $\left(X, X_{n}, X_{n-1}\right)$ to obtain a sequence $\left\{f_{n, k}\right\}_{k \in \mathbb{Z}_{+}}$in $X^{\prime}$ such that $\left\{f_{n, k}: k \in \mathbb{Z}_{+}\right\}$ is uniformly equicontinuous, each $f_{n, k}$ vanishes on $X_{n-1}$ and $\phi \subseteq\left\{\left\{f_{n, k}(x)\right\}_{k \in \mathbb{Z}_{+}}: x \in X_{n}\right\}$. According to Lemma 3.3, there exists a Banach disk $K$ in $X$ such that $X_{k}$ is a dense subspace of $X_{1}$ and the Banach space $X_{k}$ is separable. Let $\left\{U_{n}\right\}_{n \in \mathbb{N}}$ be a base of topology of $X_{k}$. We shall construct inductively a sequence of operators $S_{k} \in L\left(X, X_{k+1}\right)$ and 
vectors $y_{k} \in X_{K}$ satisfying the following conditions for any $k \in \mathbb{Z}_{+}$:

(p1) $S_{j} x=S_{k} x$ for any $j<k$ and $x \in X_{j}$;

(p2) $S_{k}\left(X_{k}\right)$ is dense in $X_{k+1}$;

(p3) $f_{k+1,0}\left(S_{k} \ldots S_{1} y_{k}\right) \neq 0$;

(p4) $S_{k} \ldots S_{1} y_{k-1}=y_{k}$ if $k \geq 2$;

(p5) $y_{k} \in U_{k}$.

Consider the linear map $S_{1}: X \rightarrow X_{2}$ defined by the formula

$$
S_{1} x=\sum_{k=0}^{\infty} 2^{-k} f_{1, k}(x) x_{2, k} \text {. }
$$

Since $\left\{x_{2, k}\right\}_{k \in \mathbb{Z}_{+}}$is an $\ell_{1}$-sequence in $X_{2}$ and $\left\{f_{1, k}: k \in \mathbb{Z}_{+}\right\}$is uniformly equicontinuous, the above display defines a continuous linear operator from $X$ to $X_{2}$. Since $\varphi \subseteq\left\{\left\{f_{1, k}(x)\right\}_{k \in \mathbb{Z}_{+}}: x \in X_{1}\right\}, S_{1}\left(X_{1}\right)$ contains span $\left\{x_{2, k}: k \in \mathbb{Z}_{+}\right\}$. Hence $S_{1}\left(X_{1}\right)$ is dense in $X_{2}$. Since $X_{k}$ is dense in $X_{1}, S_{1}$ has dense range and $X_{2} \cap \operatorname{ker} f_{2,0}$ is nowhere dense in $X_{2}$, we can pick $y_{1} \in U_{1}$ such that $f_{2,0}\left(S_{1} y_{1}\right) \neq 0$. The basis of induction has been constructed. Assume now that $n \geq 2$ and $y_{k} \in X_{K}, S_{k} \in L\left(X, X_{k+1}\right)$, satisfying (p1-p5) for $k \leq n-1$, are already constructed. According to (p3) for $k=n-1$, we have $f_{n, 0}(w) \neq 0$, where $w=S_{n-1} \ldots S_{1} y_{n-1}$. Since $H=X_{n} \cap \operatorname{ker} f_{n, 0}$ is a closed hyperplane in $X_{n}$, we have $X_{n}=H \oplus \operatorname{span}\{w\}$. Let also $H_{0}=H \cap \operatorname{ker} f_{n, 1}$. Then $H_{0}$ is a closed hyperplane of $H$. By (p2) for $k<n$, the operator $S_{n-1} \ldots S_{1}$ from $X_{1}$ to $X_{n}$ has dense range. Since $X_{K}$ is dense in $X_{1}$, we can pick $y_{n} \in U_{n}$ such that $u=S_{n-1} \ldots S_{1} y_{n} \notin H_{0} \oplus \operatorname{span}\{w\}$. Thus $X_{n}=H_{0} \oplus \operatorname{span}\{u, w\}$. From definition of $H_{0}$ it now follows that the matrix $\left(\begin{array}{ll}f_{n, 0}(w) & f_{n, 1}(w) \\ f_{n, 0}(u) & f_{n, 1}(u)\end{array}\right)$ is invertible. The latter property allows us for any two vectors $x_{0}, x_{1} \in X_{n+1}$ to find $y_{0}, y_{1} \in \operatorname{span}\left\{x_{0}, x_{1}\right\} \subset X_{n+1}$ satisfying $f_{n, 0}(w) y_{0}+f_{n, 1}(w) y_{1}=x_{0}$ and $f_{n, 0}(u) y_{0}+f_{n, 1}(u) y_{1}=x_{1}$. Pick any vector $v \in X_{n+1}$ such that $f_{n+1,0}(v) \neq 0$ and let

$$
x_{0}=y_{n}-S_{n-1} w-\sum_{k=0}^{\infty} 2^{-k} f_{n, k+2}(w) x_{n+1, k}, \quad x_{1}=v-S_{n-1} u-\sum_{k=0}^{\infty} 2^{-k} f_{n, k+2}(u) x_{n+1, k} .
$$

The above series converge since $\left\{x_{n+1, k}\right\}_{k \in \mathbb{Z}_{+}}$is an $\ell_{1}$-sequence and $\left\{f_{n, k}: k \in \mathbb{Z}_{+}\right\}$is uniformly equicontinuous. Applying the above property to the pair $x_{0}, x_{1} \in X_{n+1}$, we find $y_{0}, y_{1} \in X_{n+1}$ such that

$$
\mathrm{f}_{\mathrm{n}, 0}(\mathrm{w}) \mathrm{y}_{0}+\mathrm{f}_{\mathrm{n}, 1}(\mathrm{w}) \mathrm{y}_{1}=\mathrm{x}_{0} \operatorname{andf}_{\mathrm{n}, 0}(\mathrm{u}) \mathrm{y}_{0}+\mathrm{f}_{\mathrm{n}, 1}(\mathrm{u}) \mathrm{y}_{1}=\mathrm{x}_{1} \text {. }
$$

Consider now the linear map $S_{n}: X \rightarrow X_{n+1}$ defined by the formula

$$
S_{n} x=S_{n-1} x+f_{n, 0}(x) y_{0}+f_{n, 1}(x) y_{1}+\sum_{k=0}^{\infty} 2^{-k} f_{n, k+2}(x) x_{n+1, k} \text {. }
$$

The above display defines a continuous linear operator since $\left\{x_{n+1, k}\right\}_{k \in \mathbb{Z}_{+}}$is an $\ell_{1}$-sequence and $\left\{f_{n, k}: k \in \mathbb{Z}_{+}\right\}$is uniformly equicontinuous. From the last three displays it follows that $S_{n} w=y_{n}$ and $S_{n} u=v$. From definition of $w$ and $u$ and the relation $f_{n+1,0}(v) \neq 0$ it follows that (p3) and (p4) for $k=n$ are satisfied. Clearly (p5) for $k=n$ is also satisfied. Since each $f_{n, k}$ vanishes on $X_{n-1}$, we have from the last display that $S_{n} x=S_{n-1} x$ for any $x \in X_{n-1}$. Hence (p1) for $k=n$ is satisfied. It remains to verify (p2) for $k=n$. Let $U$ be a non-empty open subset of $X_{n+1}$. Since $E=\operatorname{span}\left\{x_{n+1, k}: k \in \mathbb{Z}_{+}\right\}$is dense in $X_{n+1}$, we can find $x \in E$ and a convex balanced neighborhood $W$ of zero in $X_{n+1}$ such that $x+W \subseteq U$. Since $\varphi \subseteq\left\{\left\{f_{n, k}(x)\right\}_{k \in \mathbb{Z}_{+}}: x \in X_{n}\right\}$, for each $x \in E=\operatorname{span}\left\{x_{n+1, k}: k \in \mathbb{Z}_{+}\right\}$, we can pick $y \in X_{n}$ such that $f_{n, 0}(y)=f_{n, 1}(y)=0$ and $x=\sum_{k=0}^{\infty} 2^{-k} f_{n, k+2}(y) x_{n+1, k}$. It follows that $S_{n} y=S_{n-1} y+x$. By (p2) for $k=n-1, S_{n-1}\left(X_{n-1}\right)$ is dense in $X_{n}$. Since $S_{n-1} y \in X_{n}$, we can find $r \in X_{n-1}$ such that $S_{n-1} r \in S_{n-1} y-W$. By the already proven property $(\mathrm{p} 1)$ for $k=n, S_{n-1} r=S_{n} r$. Hence $S_{n} r \in S_{n-1} y-W$. Using the equality $S_{n} y=S_{n-1} y+x$, we get $S_{n}(y-r) \in x+W \subseteq U$. Hence any non-empty open subset of $X_{n+1}$ contains elements of $S_{n}\left(X_{n}\right)$, which proves (p2) for $k=n$. Thus the inductive construction of $S_{k}$ and $y_{k}$ is complete.

Condition (p2) ensures that there is a unique linear map $S: X \rightarrow X$ such that $\left.S\right|_{X_{n}}=\left.S_{n}\right|_{X_{n}}$ for any $n \in \mathbb{N}$. From (p4) it now follows that $S^{k+1} y_{k}=y_{k+1}$ for each $k \in \mathbb{N}$. Thus the set $A=\left\{y_{n}: n \in \mathbb{N}\right\}$ is contained in the orbit $O\left(S, y_{1}\right)$. By (p5) $A$ is dense in $X_{k}$ and therefore is dense in $X_{1}$. By (p2) $S^{m}(A)$ is dense in $X_{m+1}$ for each $m \in \mathbb{Z}_{+}$. Since $A \subset O\left(S, y_{1}\right)$, we have that $S^{m}(A) \subset O\left(S, y_{1}\right)$ and therefore $O\left(S, y_{1}\right) \cap X_{m}$ is dense in $X_{m}$ for each $m \in \mathbb{N}$. Hence $O\left(S, y_{1}\right)$ is dense in $X$.

Before proving Theorem 1.11, we need to make the following two elementary observations.

Lemma 11.2 Let $X$ be an LB-space and $Y$ be a closed linear subspace of $X$. Then either $X / Y$ is finite dimensional or the topology of $X / Y$ is not weak.

Proof. Since $X$ is an LB-space, it is the inductive limit of a sequence $\left(X_{n},\|\cdot\|_{n}\right)$ of Banach spaces. If $X / Y$ is infinite dimensional, we can find a linearly independent sequence $\left\{f_{n}\right\}_{n \in \mathbb{Z}_{+}}$in $X^{\prime}$ such that each $f_{n}$ vanishes on $Y$. Next, we find a sequence $\left\{\varepsilon_{n}\right\}_{n \in \mathbb{Z}_{+}}$of positive numbers converging to zero fast enough to ensure that $\varepsilon_{n}\left\|\left.f_{n}\right|_{X_{k}}\right\|_{k} \rightarrow 0$ as $n \rightarrow \infty$ for each $k \in \mathbb{N}$. It follows that the sequence $\varepsilon_{n} f_{n}$ is pointwise convergent to zero on $X$. Since any LB-space is barrelled $[17,29]$, the set $\left\{\varepsilon_{n} f_{n}: n \in \mathbb{Z}_{+}\right\}$is uniformly equicontinuous. Hence $p(x)=\sup \left\{\varepsilon_{n}\left|f_{n}(x)\right|: n \in \mathbb{Z}_{+}\right\}$is a continuous seminorm on $X$. Since each $f_{n}$ vanishes on $Y, Y \subseteq \operatorname{ker} p$. Then $\tilde{p}(x+Y)=p(x)$ is a continuous seminorm on $X / Y$. Since $f_{n}$ are linearly independent $\operatorname{ker} p$ has infinite codimension in $X$ and therefore ker $\tilde{p}$ has infinite codimension in $X$ $/ Y$. Hence the topology of $X / Y$ is not weak.

Lemma 11.3 Let $X$ be an inductive limit of a sequence $\left\{X_{n}\right\}_{n \in \mathbb{Z}_{+}}$ of Banach spaces such that $X_{0}$ is dense in $X$. Then $X$ has no quotients isomorphic to $\varphi$.

Proof. Assume that $X$ has a quotient isomorphic to $\varphi$. By Lemma 9.2 then $X$ is isomorphic to $Y \times \varphi$ for some closed linear subspace $Y$ of $X$. Let $J: X_{0} \rightarrow X$ be the natural embedding. Since $X_{0}$ is dense in $X, J$ has dense range. Hence $J^{\prime}: X^{\prime} \rightarrow X_{0}^{\prime}$ is injective. Since $X$ is isomorphic 
to $Y \times \varphi$, we have that $X_{\beta}^{\prime}$ is isomorphic to $Y_{\beta}^{\prime} \times \omega$ ( $\omega$ is naturally isomorphic to $\varphi^{\prime} \beta$ ). Hence, there exists an injective continuous linear operator from $\omega$ to the Banach space $X_{0}^{\prime}$ (with the topology $\beta\left(X_{0}^{\prime}, X_{0}\right)$ ). That is impossible, since any injective continuous linear operator from $\omega$ to a locally convex space is an isomorphism onto image and $\omega$ is non-normable.

\section{Proof of Theorem 1.11}

Throughout this section $X$ is the inductive limit of a sequence $\left\{X_{n}\right\}_{n \in \mathbb{Z}_{+}}$of separable Banach spaces. Let also $\bar{X}_{n}$ be the closure of $X_{n}$ in $X$. First, we shall prove the implication $(1.11 .4) \Rightarrow(1.11 .3)$. Assume that (1.11.4) is satisfied. Then we can pick a strictly increasing sequence $\left\{n_{k}\right\}_{k \in \mathbb{Z}_{+}}$of non-negative integers such that $0<\operatorname{dim} \bar{X}_{n_{k+1}} / \bar{X}_{n_{k}}<\infty$ for each $k \in \mathbb{Z}_{+}$. Hence, for any $k \in \mathbb{Z}_{+}$, we can pick a non-trivial finite dimensional subspace $Y_{k}$ of $X_{n_{k+1}}$ such that $\bar{X}_{n_{k}} \oplus Y_{k}=\bar{X}_{n_{k+1}}$. Thus the vector space $X$ can be written as an algebraic direct sum

$$
X=\overline{X_{n_{0}}} \oplus \bigoplus_{k=0}^{\infty} Y_{k} .
$$

Apart from the original topology $\tau$ on $X$, we can consider the topology $\theta$, turning the sum (11.1) into a locally convex direct sum. Obviously $\tau \subseteq \theta$. On the other hand, if $W$ is a balanced convex $\theta$-neighborhood of 0 in $X$, then $W \cap \overline{X_{n_{k}}}$ is a $\tau$-neighborhood of zero in $\overline{X_{n_{k}}}$ for any $k \in \mathbb{Z}_{+}$. Indeed, it follows from the fact that $\overline{X_{n_{k}}}=\overline{X_{n_{0}}} \oplus Z_{k}$, where $Z_{k}=\bigoplus_{j=0}^{k-1} Y_{j}$ and $Z_{k}$ is finite dimensional. Since the topology of each is stronger than the one inherited from $X$, we see that $W \cap X_{n_{k}}$ is a neighborhood of zero in $X_{n_{k}}$ for each $k \in$ $\mathbb{Z}_{+}$. Since $X$ is the inductive limit of the sequence $\left\{X_{n_{k}}\right\}_{k \in \mathbb{Z}_{+}}, W$ is a $\tau$ -neighborhood of zero in $X$. Hence $\theta \subseteq \tau$. Thus $\theta=\tau$ and therefore $X$ is isomorphic to $\bar{X}_{n_{0}} \times Y$, where $Y$ is the locally convex direct sum of $Y_{k}$ for $k \in \mathbb{Z}_{+}$. Since $Y_{k}$ are finite dimensional, $Y$ is isomorphic to $\varphi$. Since $\bar{X}_{n_{0}}$ is the inductive limit of the sequence $\left\{\bar{X}_{n_{0}} \cap X_{n_{k}}\right\}_{k \in \mathbb{Z}_{+}}$of separable Banach spaces (with the topology inherited from $X_{n_{k}}$ ), the first one $X_{n_{0}}$ of which is dense, we see that (1.11.3) is satisfied. The implication $(1.11 .4) \Rightarrow(1.11 .3)$ is verified.

Assume now that (1.11.3) is satisfied. By Lemma 11.3, $Y$ has no quotients isomorphic to $\varphi$. Theorem 1.12 implies now that there are no cyclic operators with dense range on $X$, which proves the implication $(1.11 .3) \Rightarrow(1.11 .2)$. The implication $(1.11 .2) \Rightarrow(1.11 .1)$ is obvious since any hypercyclic operator is cyclic and has dense range. It remains to show that (1.11.1) implies (1.11.4). Assume the contrary. That is, (1.11.1) is satisfied and (1.11.4) fails. The latter implies that either there is $n \in \mathbb{Z}_{+}$such that $\bar{X}_{n}$ is dense in $X$ or there is a strictly increasing sequence $\left\{n_{k}\right\}_{k \in \mathbb{Z}_{+}}$of non-negative integers such that $\bar{X}_{n_{k+1}} / \bar{X}_{n_{k}}$ is infinite dimensional for each $k \in \mathbb{Z}_{+}$. In the first case, it is easy to see that $X \in \mathfrak{M}$ and therefore there is a hypercyclic operator on $X$ by Corollary 1.4. We have obtained a contradiction with (1.11.1). It remains to consider the case when there exists a strictly increasing sequence $\left\{n_{k}\right\}_{k \in \mathbb{Z}_{+}}$of non-negative integers such that $\bar{X}_{n_{k}} \quad \bar{X}_{n_{k}}$ is infinite dimensional for each $k \in \mathbb{Z}_{+}$. By Lemma 11.2, the topology of each $\bar{X}_{n_{k+1}} / \bar{X}_{n_{k}}$ is not weak. Let $k \in \mathbb{Z}_{+}$. Since $X_{n_{k}}$ is a separable Banach space, there is an $\ell_{1}$-sequence $\left\{x_{k, j}\right\}_{j \in \mathbb{Z}_{+}}$in $X_{n_{k}}$ with dense span. Since the topology on $X_{n_{k}}$ inherited from $X$ is weaker than the Banach space topology of $X_{n_{k}},\left\{x_{k, j}\right\}_{j \in \mathbb{Z}_{+}}$is an $\ell_{1}$-sequence with dense span in $\bar{X}_{n_{k}}$. By Lemma 11.1, there exists a linear map $S: X \rightarrow X$ and $x_{0} \in X$ such that for any $k \in \mathbb{Z}_{+}$, the restriction of $S$ to $\bar{X}_{n_{k}}$ is continuous and the orbit $O(S, x)=\left\{S^{k} x: k \in \mathbb{Z}_{+}\right\}$is dense in $X$. Since the topology of $X_{n_{k}}$ is stronger than the one inherited from $X$, we have that each restriction of $S$ to $X_{n_{k}}$ is a continuous linear operator from $X_{n_{k}}$ to $X$. Since $X$ is the inductive limit of the sequence $\left\{X_{n_{k}}\right\}_{k \in \mathbb{Z}_{+}}$ , $S: X \rightarrow X$ is continuous. Hence $S$ is a hypercyclic continuous linear operator on $X$. The existence of such an operator contradicts (1.11.1). The proof of the implication $(1.11 .1) \Rightarrow(1.11 .4)$ and that of Theorem 1.11 is now complete.

\section{Remarks on mixing versus hereditarily hypercyclic}

We start with the following remark. As we have already mentioned, $\varphi$ supports no supercyclic operator [11], which follows also from Theorem 1.12. On the other hand, $\varphi$ supports a transitive operator [22]. The latter statement can be easily strengthened with the help of Corollary 5.1. Namely, take the backward shift $T$ on $\varphi$. That is $T e_{0}=0$ and $T e_{n}=e_{n-1}$ for $n \geq 1$, where $\left\{e_{n}\right\}_{n \in \mathbb{Z}_{+}}$is the standard basis in $\varphi$. Clearly $T$ is a generalized backward shift and therefore $T$ is an extended backward shift. By Corollary 5.1, $I+T$ is mixing. Thus we have the following proposition.

Proposition 12.1 $\varphi$ supports a mixing operator and supports no supercyclic operators.

On the other hand, a topological vector space of countable algebraic dimension can support a hypercyclic operator, as observed by several authors, [22], for instance. The following proposition formalizes and extends this observation.

Proposition 12.2 Let $X$ be a normed space of countable algebraic dimension. Then there exists a hypercyclic mixing operator $T \in L(X)$.

Proof. Let $\bar{X}$ be the completion of $X$. Then $\bar{X}$ is a separable infinite dimensional Banach space. By Corollary 1.4, there is a hereditarily hypercyclic operator on $S \in L(\bar{X})$. Let $x \in \bar{X}$ be a hypercyclic vector for $S$ and $E$ be the linear span of the orbit of $x: E=\operatorname{span}\left\{S^{n} x: n \in \mathbb{Z}_{+}\right\}$. Grivaux [39] demonstrated that for any two countably dimensional dense linear subspaces $E$ and $F$ of a separable infinite dimensional Banach space $Y$, there is an isomorphism $J: Y \rightarrow Y$ such that $J(E)=F$. Hence there is an isomorphism $J: \bar{X} \rightarrow \bar{X}$ such that $J(X)=E$. Let now $T_{0}=J^{-1} S J$. Since $J(X)=E$ and $E$ is $S$-invariant, $X$ is $T_{0}$-invariant. Thus the restriction $T$ of $T_{0}$ to $X$ is a continuous linear operator on $X$. Moreover, since the $S$-orbit of $s$ is dense in $\bar{X}$, the $T_{0}$-orbit of $J^{-1} x$ is dense in $\bar{X}$. Since $J^{-1} x \in X$, the latter orbit is exactly the $T$-orbit of $J^{-1} x$ and therefore $J^{-1} x$ is hypercyclic for $T$. Hence $T$ is hypercyclic. Next, $T_{0}$ is mixing since it is similar to the mixing operator $S$. Hence $T$ is mixing as a restriction of a mixing operator to a dense subspace.

By Proposition 1.1, if $X$ is a Baire separable and metrizable topological vector space, then any mixing $T \in L(X)$ is hereditarily hypercyclic. From the above proposition it follows that there are mixing 
operators on countably dimensional normed spaces. The next theorem however implies that there are no hereditarily hypercyclic operators on countably dimensional topological vector spaces, emphasizing the necessity of the Baire condition in Proposition 1.1.

Theorem 12.3 Let $X$ be a topological vector space such that there exists a hereditarily universal family $\left\{T_{n}: n \in \mathbb{Z}_{+}\right\} \subset L(X)$. Then $\operatorname{dim} X>\aleph_{0}$

Proof. Since the topology of any topological vector space can be defined by a family of quasinorms [17], we can pick a nonzero continuous quasinorm $P$ on $X$. That is, $p: X \rightarrow[0, \infty)$ is nonzero, continuous, $p(x+y) \leq p(x)+p(y)$ for any $x, y \in X, p(0)=0$, $p(z x)=p(x)$ if $x \in X, z \in \mathbb{K},|z|=1$ and $\left(X, \tau_{p}\right)$ is a (not necessarily Hausdorff) topological vector space, where $\tau_{p}$ is the topology defined by the pseudometric $d(x, y)=p(x-y)$. The latter property implies that $p\left(t x_{n}\right) \rightarrow 0$ for any $t \in \mathbb{K}$ and any sequence $\left\{x_{n}\right\}_{n \in \mathbb{Z}_{+}}$in $X$ such that $p\left(x_{n}\right) \rightarrow 0$.

Let $\kappa$ be the first uncountable ordinal (commonly denoted $\omega_{1}$ ). We shall construct inductively sequences $\left\{x_{a}\right\}_{\alpha<\kappa}$ and $\left\{A_{a}\right\}_{\alpha<\kappa}$ of vectors in $X$ and subsets of $\mathbb{Z}_{+}$respectively such that for any $\alpha<\kappa$, pt

(s1) $A_{\alpha}$ is infinite and $x_{\alpha}$ is a universal vector for the family $\left\{T_{n}: n\right.$ $\left.\in A_{\alpha}\right\}$;

(s2) $p\left(T_{n} x_{\beta}\right) \rightarrow 0$ as $n \rightarrow \infty, n \in A_{\alpha}$ for any $\beta<\alpha$;

(s3) $A_{\alpha} \backslash A_{\beta}$ is finite for any $\beta<\alpha$.

For the basis of induction we take $A_{0}=\mathbb{Z}_{+}$and $x_{0}$ being a universal vector for the family $\left\{T_{n}: n \in \mathbb{Z}_{+}\right\}$. It remains to describe the induction step. Assume that $\gamma<\kappa$ and $x_{\alpha}, A_{\alpha}$ satisfying (s1-s3) for $\alpha<\gamma$ are already constructed. We have to construct $x_{\gamma}$ and $A_{\gamma}$ satisfying (s1-s3) for $\alpha=\gamma$.

Case 1: $\gamma$ has the immediate predecessor. That is $\gamma=\rho+1$ for some ordinal $\rho<\kappa$. Since $x_{p}$ is universal for $T_{n}: n \in A_{\rho}$, we can pick an infinite subset $A_{\gamma} \subset A_{\rho}$ such that $p\left(T_{n} x_{\rho}\right) \rightarrow 0$ as $n \rightarrow \infty, n \in A_{\gamma}$ . Since $A_{\gamma}$ is contained in $A_{p}$, from (s3) for $\alpha \leq \rho$ it follows that $A_{\gamma} \backslash A_{\beta}$ is finite for any $\beta<\gamma$. Hence (s3) for $\alpha=\gamma$ is satisfied. Now from (s3) for $\alpha=\gamma$. and (s2) for $\alpha<\gamma$. it follows that (s2) is satisfied for $\alpha=\gamma$. Next, since $\left\{T_{n}: n \in \mathbb{Z}_{+}\right\}$is hereditarily universal, we can pick $x_{\gamma} \in X$ universal for $\left\{T_{n}: n \in A_{\gamma}\right\}$. Hence (s1) for $\alpha=\gamma$. is also satisfied.

Case 2: $\gamma$ is a limit ordinal. Since $\gamma$ is a countable ordinal, we can pick a strictly increasing sequence $\left\{\alpha_{n}\right\}_{n \in \mathbb{Z}_{+}}$of ordinals such that $\gamma=\sup \left\{\alpha_{n}: n \in \mathbb{Z}_{+}\right\}$. Now pick consecutively $n_{0}$ from $A_{\alpha_{0}}, n_{1}>n_{0}$ from $A_{\alpha_{0}} \cap A_{\alpha_{1}}, n_{2}>n_{1}$ from $A_{\alpha_{0}} \cap A_{\alpha_{1}} \cap A_{\alpha_{2}}$ etc. The choice is possible since by (s3) for $\alpha<\gamma$, each $A_{\alpha_{0}} \cap \ldots \cap A_{\alpha_{n}}$ is infinite. Now let $A_{\gamma}=\left\{n_{j}: j \in \mathbb{Z}_{+}\right\}$. Since $A_{\gamma} \backslash A_{\alpha_{j}} \subseteq\left\{n_{0}, \ldots, n_{j-1}\right\}, A_{\gamma} \backslash A_{\alpha_{j}}$ is finite for each $j \in \mathbb{Z}_{+}$. Now if $\beta<\gamma$, we can pick $j \in \mathbb{Z}_{+}$such that $\beta<\alpha_{j}$ $<\gamma$. Then $A_{\gamma} \backslash A_{\beta} \subseteq\left(A_{\gamma} \backslash A_{\alpha_{j}}\right) \cup\left(A_{\alpha_{j}} \backslash A_{\beta}\right)$ is finite by (s3) with $\alpha={ }_{j}$. Moreover, since $A_{\gamma}$ is contained in $A_{\alpha_{j}}$ up to a finite set, from (s2) with $\alpha=\alpha_{j}$ it follows that $p(T x) \rightarrow 0$ as $n \rightarrow \infty, n \in A_{\gamma}$. Hence (s2) and (s3) for $\alpha=\gamma$ are satisfied. Finally, since $\left\{T_{n}: n \in \mathbb{Z}_{+}\right\}$is hereditarily universal, we can pick $x_{\gamma} \in X$ universal for $\left\{T_{n}: n \in A_{\gamma}\right\}$. Hence (s1) for $\alpha=\gamma$ is also satisfied. This concludes the construction of $\left\{x_{a}\right\}_{\alpha<\kappa}$ and $\left\{A_{a}\right\}_{\alpha<\kappa}$ satisfying (s1-s3).

In order to prove that $\operatorname{dim} X>\aleph_{0}$, it suffices to show that vectors $\left\{x_{a}\right\}_{\alpha<\kappa}$ are linearly independent. Assume the contrary. Then there are $n \in \mathbb{N}, z_{1}, \ldots, z_{n} \in \mathbb{K} \backslash\{0\}$ and ordinals $\alpha_{1}<\ldots<\alpha_{n}<\kappa$ such that $\sum_{j=1}^{n} z_{j} x_{\alpha_{j}}=0$. By (s2) with $\alpha=\alpha_{n}$, we see that $p\left(T_{k} x_{\alpha_{j}}\right) \rightarrow 0$ as $k \rightarrow \infty$, $k \in A_{\alpha_{n}}$ for $1 \leq j<n$. Denoting $c_{j}=-z_{j} / z_{n}$ for $1 \leq j<n$ and using linearity of $T_{k}$, we obtain $T_{k} x_{\alpha_{n}}=\sum_{1 \leqslant j<n} c_{j} T_{k} x_{\alpha_{j}}$ for any $k \in \mathbb{Z}_{+}$. Since $p$ is a quasinorm, we have

$$
p\left(T_{k} x_{\alpha_{n}}\right) \leqslant \sum_{1 \leqslant j<n} p\left(c_{j} T_{k} x_{\alpha_{j}}\right) \rightarrow 0 \text { ask } \rightarrow \infty, \mathrm{k} \in \mathrm{A}_{\alpha_{\mathrm{n}}} .
$$

The above display contradicts universality of $x_{\alpha_{n}}$ for $\left\{T_{k}: k \in A_{\alpha_{n}}\right\}$, which is (s1) with $\alpha=\alpha_{n}$. This contradiction completes the proof.

Corollary 12.4 A topological vector space of countable algebraic dimension supports no hereditarily hypercyclic operators.

It is worth noting that there are infinite dimensional separable normed spaces, which support no supercyclic or transitive operators. We call a continuous linear operator $T$ on a topological vector space $X$ simple if $T$ has shape $T=z I+S$, where $z \in \mathbb{K}$ and $S$ has finite rank. Observe that a simple operator on an infinite dimensional topological vector space is never transitive or supercyclic. Indeed, let $T$ be a simple operator on an infinite dimensional topological vector space and $\lambda \in \mathbb{K}, S \in L(X)$ be such that $T=\lambda I+S$ and $S$ has finite rank. Then $L=S(X)$ is finite dimensional. Since $X / Y$ is infinite dimensional, we can pick non-empty open subsets $U_{0}$ and $V_{0}$ of $X / L$ such that $U_{1}=\left\{z u: z \in \mathbb{K} \backslash\{0\}, u \in U_{0}\right\}$ does not intersect $V_{1}=\left\{z v: z \in \mathbb{K} \backslash\{0\}, v \in V_{0}\right\}$. Let $U=\left\{z x: x+L \in U_{0}, z \in \mathbb{K} \backslash\{0\}\right\}$ and $V=\left\{z x: x+L \in V_{0}, z \in \mathbb{K} \backslash\{0\}\right\}$. Clearly $U$ and $V$ are non-empty open subsets of $X$. Using the equalities $T=\lambda I+S$ and $S(X)=L$, it is easy to see that $T^{n}(U) \cap V=\varnothing$ for any $n \in \mathbb{Z}_{+}$. Hence $T$ is non-transitive. Moreover since $U$ and $V$ are stable under multiplication by non-zero scalars, the projective orbit $\left\{z T^{n} x: n \in \mathbb{Z}_{+}, z \in \mathbb{K}\right\}$ of any $x \in U$ does not meet $V$. Hence $U$ contains no supercylic vectors for $T$. Since the set of supercyclic vectors of any continuous linear operator is either dense or empty, $T$ is non-supercyclic.

We say that a topological vector space $X$ is simple if it is infinite dimensional and any $T \in L(X)$ is simple. Thus simple topological vector spaces support no supercyclic or transitive operators. Various examples of simple separable infinite dimensional normed spaces can be found in the literature $[38,40-44]$. Moreover, according to Valdivia [40], in any separable infinite dimensional Fréche spaced there is a dense simple hyperplane. All the examples of this type existing in the literature with one exception [44] are constructed with the help of the axiom of choice and the spaces produced are not Borel measurable in their completions. In [44] there is a constructive example of a simple separable infinite dimensional pre-Hilbert space $H$ which is a countable union of compact sets.

Finally recall that an infinite dimensional topological vector space $X$ is called rigid if $L(X)$ consists only of the operators of the form $I$ for $\lambda \in \mathbb{K}$. Of course, a rigid space can not be locally convex. Clearly there are no transitive or cyclic continuous linear operators on a rigid topological vector space. Since there exist rigid separable $\mathcal{F}$-spaces [45], we see that there are separable infinite dimensional $\mathcal{F}$-spaces on which there are no cyclic or transitive operators.

\section{Concluding remarks and open problems}

We start by observing that the following questions remain open. 
Problem 13.1 Is there a hereditarily hypercyclic operator on a countable direct sum of separable infinite dimensional Banach spaces?

Problem 13.2 Is there a hypercyclic strongly continuous operator semigroup on a countable direct sum of separable infinite dimensional Banach spaces?

The most of the above results rely upon the underlying space being locally convex or at least having plenty of continuous linear functionals and for a good reason. As mentioned in the previous section, there are separable infinite dimensional $\mathcal{F}$-spaces on which there are no cyclic or transitive operators. On the other hand, the absence of non-zero continuous linear functionals on a topological vector space does not guarantee the absence of hypercyclic operators on it. It is well-known [45] that the spaces $L_{p}[0,1]$ for $0 \leq \mathrm{p}<1$ are separable $\mathcal{F}$-spaces having no non-zero continuous linear functionals. Ansari [9] raises a question whether these spaces support hypercyclic operators. Theorem 1.18 provides an easy answer to this question. Namely, consider the operator $T \in L\left(L_{p}[0,1]\right), T f(x)=f(x / 2)$. It is straightforward to see that $T$ is onto and has dense generalized kernel. Thus $I+T$ is hereditarily hypercyclic according to Corollary 5.2.

It is obvious that an extended backward shift has dense range and dense generalized kernel. Unfortunately, the converse is not true in general. This leads naturally to the following question.

Problem 13.3 Let $T$ be a continuous linear operator on a separable Banach space, which has dense range and dense generalized kernel. Is it true that $I+T$ is mixing or at least hypercyclic?

From Corollary 5.1 and Corollary 2.14 it follows that if $T$ is an extended backward shift on a separable infinite dimensional Banach space $X$, then both $I+T$ and $e^{T}$ are hereditarily hypercyclic. This reminds of the following question raised by Bermúdez, Bonilla, Conejero and Peris in reference [15].

Question $B^{2}$ CP. Let $X$ be a complex Banach space and $T \in L(X)$ be such that its spectrum $\sigma(T)$ is connected and contains 0 . Does hypercyclicity of I+T imply hypercyclicity of $e^{T}$ ? Does hypercyclicity of $e^{T}$ imply hypercyclicity of $I+T$ ?

We show that the answer to both parts of the above question is negative. Before doing this we would like to raise a similar question, which remains open. $I+T$

Problem 13.4 Let $X$ be a Banach space and $T \in L(X)$ be quasinilpotent. Is hypercyclicity of I+T equivalent to hypercyclicity of $e^{T}$ ?

First, we introduce some notation. Let $\mathbb{D}=\{z \in \mathbb{C}:|z|<1\}, \mathcal{H}^{2}(\mathbb{D})$ be the Hardy Hilbert space on the unit disk and $\mathcal{H}^{\infty}(\mathbb{D})$ be the space of bounded holomorphic functions $f: \mathbb{D} \rightarrow \mathbb{C}$. For any $\varphi \in \mathcal{H}^{\infty}(\mathbb{D})$, the multiplication operator

$$
M_{\varphi} f(z)=\varphi(z) f(z)
$$

is a bounded linear operator on $\mathcal{H}^{2}(\mathbb{D})$. It is also clear that $\sigma\left(M_{\varphi}\right)=\overline{\varphi(\mathbb{D})}$. If $M_{\varphi}^{*}$ is the adjoint of $M \varphi$, then $\sigma\left(M_{\varphi}^{*}\right)$ is the reflection of $\sigma\left(M_{\varphi}\right)$ with respect to the real axis. The following proposition is a direct consequence of a theorem by Godefroy and Shapiro [27][Theorem 4.9]. only if

Proposition 13.5 Let $\varphi \in \mathcal{H}^{\infty}(\mathbb{D})$. Then $M_{\varphi}^{*}$ is hypercyclic if and

$\varphi(\mathbb{D}) \cap \mathbb{T} \neq \varnothing$.

The above Proposition calls for the following comment. A bounded linear operator $T$ on a separable infinite dimensional Banach space $X$ is said to satisfy the Kitai Criterion [46,47] if there exist dense subsets $E$ and $F$ of $X$ and a map $S: F \rightarrow F$ such that $T S y=y$ for any $y \in F, T^{n} x \rightarrow 0$ and $S^{n} y \rightarrow 0$ as $n \rightarrow \infty$ for any $x \in E$ and $y \in F$. As it is shown in [46,47], any operator satisfying the Kitai Criterion is hypercyclic. Moreover, any operator, satisfying the Kitai Criterion is hereditarily hypercyclic and therefore mixing [3]. Hypercyclicity in the proof of the above result in reference [27] is demonstrated via application of the Kitai Criterion. Thus the following slightly stronger statement holds.

Corollary 13.6 Let $\varphi \in \mathcal{H}^{\infty}(\mathbb{D})$. Then $M_{\varphi}^{*}$ is hereditarily hypercyclic if (13.1) is satisfied and $M_{\varphi}^{*}$ is non-hypercyclic if (13.1) is not satisfied.

Now we demonstrate that the answer to both parts of Question $\mathrm{B}^{2} \mathrm{CP}$ is negative. Consider the subset $U$ of $\mathbb{C}$ being the interior of the triangle with vertices $-1, I$ and $-i$. In other words $U=\{a+b i: a, b \in \mathbb{R}, a<0, b-a<1, b+a>-1\} . \quad$ Next, let $V=\left\{a+b i: a, b \in \mathbb{R}, 0<b<1,|a|<1-\sqrt{1-b^{2}}\right\}$. The boundary of $V$ consists of the interval $[-1+i, 1+i]$ and two circle arcs. It is clear that $U$ and $V$ are bounded, open, connected and simply connected. From the definition of the sets $U$ and $V$ it immediately follows that the open set $1+U=\{1+z: z \in U\}$ intersects the unit circle. On the other hand, since $U$ is contained in the left half-plane, we see that $e^{U}=\left\{e^{z}: z \in U\right\} \subseteq \mathbb{D}$. Similarly, we see that $1+V \cap \mathbb{D}=\varnothing$ and the open set $e^{V}$ intersects the unit circle. According to the Riemann Theorem [48], there exist holomorphic homeomorphisms $\phi: \mathbb{D} \rightarrow U$ and $\psi: \mathbb{D} \rightarrow V$. Obviously $\varphi, \psi \in \mathcal{H}^{\infty}(\mathbb{D})$. Since $I+M_{\varphi}^{*}=M_{1+\varphi}^{*}, \quad e^{M_{\psi}^{*}}=M_{e^{\psi}}^{*} \quad$ and both $(1+\phi)(\mathbb{D})=1+U$ and $e^{\psi}(\mathbb{D})=e^{V}$ intersect the unit circle, Corollary 13.6 implies that $I+M_{\varphi}^{*}$ and $e^{M_{\psi}^{*}}$ are hereditarily hypercyclic. Since $I+M_{\psi}^{*}=M_{1+\psi}^{*}, e^{M_{\varphi}^{*}}=M_{e^{\varphi}}^{*}, e^{\varphi}(\mathbb{D})=e^{U}$ is contained in $\mathbb{D}$, and $(1+\psi)(\mathbb{D})=1+V$ does not intersect $\overline{\mathbb{D}}$, Corollary 13.6 implies that $e^{M_{\varphi}^{*}}$ and $I+M_{\psi}^{*}$ are non-hypercyclic. Finally, observe that $\sigma\left(M_{\varphi}^{*}\right)$ is the closure of $U$ and $\sigma\left(M_{\psi}^{*}\right)$ is the closure of $-V$ and therefore the spectra of $M_{\varphi}^{*}$ and $M_{\psi}^{*}$ are connected and contain 0 . Taking into account that all separable infinite dimensional Hilbert spaces are isomorphic, we arrive to the following result, which answers negatively the Question $\mathrm{B}^{2} \mathrm{CP}$.

Proposition 13.7 There exist bounded linear operators $A, B$ on the complex Hilbert space $\ell_{2}$ such that $\sigma(A)$ and $\sigma(B)$ are connected and contain $0, I+A$ and $e^{B}$ are hereditarily hypercyclic, while $e^{A}$ and $I+B$ are non-hypercyclic.

Finally, if the answer to Question 13.4 is affirmative, then the following interesting question naturally arises.

Problem 13.8 Let A be a quasinilpotent bounded linear operator on a complex Banach space $X$ and $f$ be an entire function on one variable such that $f(0)=f^{\prime}(0)=1$. Is it true that hypercyclicity of $f(A)$ is equivalent to hypercyclicity of $I+A$ ?

\section{Spaces $C_{k}(M)$ and their duals}

Let $(M, d)$ be a separable metric space and $C_{k}(M)$ be the space of continuous functions $f: M \rightarrow \mathbb{K}$ with the compact-open topology 
(=the topology of uniform convergence on compact subsets of $M$ ). It is easy to see that $C_{k}(M)$ is complete. Moreover, $C_{k}(M)$ is metrizable if and only $M$ is locally compact and $C_{k}(M)$ carries weak topology if and only if $M$ is discrete. On the other hand, there always is an $\ell_{1}$ sequence with dense span in $C_{k}(M)$. Indeed, let $\bar{M}$ be a metrizable compactification of $M$. Since $C(\bar{M})$ is a separable Banach space, there is an $\ell_{1}$-sequence $\left\{f_{n}\right\}_{n \in \mathbb{Z}_{+}}$with dense span in $C(\bar{M})$. Since $C(\bar{M})$ is densely and continuously embedded into $C_{k}(M),\left\{f_{n}\right\}_{n \in \mathbb{Z}}$ is an $\ell_{1}$ sequence with dense span in $C_{k}(M)$. Thus $C_{k}(M) \in \mathfrak{M}$ if and only if $M$ is non-discrete. Corollary 1.4 implies now the following proposition.

Proposition 13.9 If $(M, d)$ is a separable non-discrete metric space, then there is a hereditarily hypercyclic operator on $C_{k}(X)$.

The reason for inclusion of the above proposition is to demonstrate that Theorem 1.3 and Corollary 1.4 are applicable far beyond metrizable or LB-spaces. The spaces $C_{k}(M)$ can have quite ugly structure indeed. For instance, take $M$ being the set $\mathbb{Q}$ of rational numbers with the metric induced from $\mathbb{R}$, and you have got the space $C_{k}(\mathbb{Q})$, which does not fall into any of the well-understood and studied classes of locally convex spaces. We would like to raise the following question.

Problem 13.10 Characterize separable metric spaces $(M, d)$ such that $C_{k}(M)$ supports a dual hypercyclic operator.

It is worth noting that if $M$ is discrete, then either $C_{k}(M)$ is finite dimensional or is isomorphic to $\omega$ and therefore does not support a dual hypercyclic operator (there are no hypercyclic operators on $\varphi=\omega^{\prime}$ ). In general, $\mathfrak{M}(M)=\left(C_{k}(M)\right)^{\prime}$ can be naturally identified with the space of finite $\mathbb{K}$-valued Borel $\sigma$-additive measures on $M$ with compact support. This dual space is separable in the strong topology if and only if all compact subsets of $M$ are finite or countable. If it is not the case, there is no point to look for dual hypercyclic operators on $C_{k}(M)$. Thus the only spaces $(M, d)$ for which $C_{k}(M)$ has a chance to support a dual hypercyclic operator are non-discrete spaces with no uncountable compact subsets. The first natural candidate to consider is $\mathbb{Q}$.

Note also that although Theorem 1.15 provides answers to the questions of Petersson, mentioned in the introduction, it does not characterize Fréchet spaces, supporting a dual hypercyclic operator.

Problem 13.11 Characterize Fréchet spaces $X$ such that $X$ supports a dual hypercyclic operator.

The most natural Fréchet space for which we do not know whether it supports a dual hypercyclic operator is the countable power $\ell_{2}^{\mathbb{N}}$ of the Hilbert space $\ell_{2}$.

\section{The Hypercyclicity Criterion}

The following universality criterion is proved by Bés and Peris [2, Theorem 2.3 and Remark 2.6]. It is formulated in [2] in the case when $X$ is an $\mathcal{F}$-space, but the proof works without any changes for Baire separable metrizable topological vector spaces.

Theorem BP Let $\left\{T_{n}\right\}_{n \in \mathbb{Z}}$ be a sequence of pairwise commuting continuous linear operators with dense range on a Baire separable metrizable topological vector space $X$. Then the following conditions are equivalent:

(a) The family $\left\{T_{n} \oplus T_{n}\right\}_{n \in \mathbb{Z}_{+}}$is universal;

(b)There exists an infinite subset $A$ of $\mathbb{Z}_{+}$such that the family $\left\{T_{n}\right\}_{n \in A}$ is hereditarily universal,

(c)There exist a strictly increasing sequence $\left\{n_{k}\right\}$ of non-negative integers, dense subsets $E$ and $F$ of $X$ and maps $S_{k}: F \rightarrow X$ for $k \in \mathbb{Z}_{+}$ such that $T_{n_{k}} x \rightarrow 0, S_{k} y \rightarrow 0$ and $T_{n_{k}} S_{k} y \rightarrow y$ as $k \rightarrow \infty$ for any $x \in$ $E$ and $y \in F .^{k}$

We formulate now the so-called Hypercyclicity and Supercyclicity Criteria, which follow easily from the above theorem.

Theorem HC Let X be a Baire separable metrizable topological vector space and $T \in L(X)$. Then the following conditions are equivalent:

(a) $T \oplus T$ is hypercyclic;

(b)There exists an infinite subset $A$ of $\mathbb{Z}_{+}$such that the family $\left\{T^{n}\right\}_{n \in A}$ is hereditarily universal;

(c)There exist a strictly increasing sequence $\left\{n_{k}\right\}$ of non-negative integers, dense subsets $E$ and $F$ of $X$ and maps $S_{k}: F \rightarrow X$ for $k \in \mathbb{Z}_{+}$ such that $T^{n} k_{x} \rightarrow 0, S_{k} y \rightarrow 0$ and $T^{n}{ }^{n} S_{k} y \rightarrow y$ as $k \rightarrow \infty$ for any $x$ $\in E$ and $y \in F$.

Theorem SC. Let $X$ be a Baire separable metrizable topological vector space and $T \in L(X)$. Then the following conditions are equivalent:

(a) $T \oplus T$ is supercyclic;

(b) There exists an infinite subset $A$ of $\mathbb{Z}_{+}$and a sequence $\left\{s_{n}\right\}_{n \in A}$ of positive numbers such that the family $\left\{s_{n} T^{n}\right\}_{n \in A}$ is hereditarily universal;

(c) There exist a strictly increasing sequence $\left\{n_{k}\right\}$ of non-negative integers, dense subsets $E$ and $F$ of $X$, and a sequence $\left\{s_{k}\right\}_{k \in \mathbb{Z}_{+}}$of positive numbers and maps $S_{k}: F \rightarrow X$ for $k \in \mathbb{Z}_{+}$such that $s_{k} T^{n} k_{x \rightarrow 0}$, $s_{k}^{-1} S_{k} y \rightarrow 0$ and $T^{n}{ }^{n} S_{k} y \rightarrow y$ as $k \rightarrow \infty$ for any $x \in E$ and $y \in F$.

An operator satisfying the condition (c) of Theorem HC (respectively Theorem SC) is said to satisfy the Hypercyclicity (respectively, Supercyclicity) Criterion. The long standing question whether any hypercyclic operator $T$ on a Banach space satisfies the Hypercyclicity Criterion, was recently solved negatively by Read and De La Rosa [49]. Their result was extended by Bayart and Matheron [50], who demonstrated that on any separable Banach space with an unconditional Schauder basis such that the forward shift operator associated with this basis is bounded, there is a hypercyclic operator $T$ such that $T \oplus T$ is not hypercyclic. This leaves open the following question raised in reference [50].

Problem 13.12 Does there exist a separable infinite dimensional Banach space $X$ such that any hypercyclic operator on $X$ satisfies the Hypercyclicity Criterion?

It is observed in reference [50] that any $T \in L(\omega)$ satisfies the Hypercyclicity Criterion. It also follows from Theorem 1.7 and Theorem HC. Thus the above question in the class of Fréchet spaces has an affirmative answer, which leads to the following problem.

Problem 13.13 Characterize separable infinite dimensional Fréchet spaces $X$ on which any hypercyclic operator on $X$ satisfies the Hypercyclicity Criterion?

It is worth noting that non-hypercyclicity of $T \oplus T$ in references $[49,50]$ is ensured by the existence of a non-zero continuous bilinear form $b: X \times X \rightarrow \mathbb{K}$ with respect to which $T$ is symmetric: $b(T x, y)=b(x, T y)$ for any $x, y \in X$. The following proposition formalizes the corresponding implication. Similar statements have been proved by many authors in various particular cases. The proof goes along the same lines as in any of them. 
Let $X$ and $Y$ be topological vector spaces and $b: X \times X \rightarrow Y$ be separately continuous and bilinear. We say that $T \in L(X)$ is $b$-symmetric if $b(T x, y)=b(x, T y)$ for any $x, y \in X$. Recall also that $b$ is called symmetric if $b(x, y)=b(y, x)$ for any $x, y \in X$ and $b$ is called antisymmetric if $b(x, y)=-b(y, x)$ for any $x, y \in X$.

Proposition 13.14 Let $X$ and $Y$ be a topological vector spaces, $b: X \times X \rightarrow Y$ be separately continuous, non-zero and bilinear and $T \in$ $L(X)$ be b-symmetric. Then $T \oplus T$ is non-cyclic. If additionally $b$ is nonsymmetric, then $T^{2}$ is non-cyclic.

Proof. Consider the left and right kernels of $b$ :

$X_{0}=\{x \in X: b(x, y)=0$ foranyy $\in \mathrm{X}\}$ and $X_{1}=\{y \in X: b(x, y)=0$ foranyx $\in \mathrm{X}\}$.

Separate continuity of $b$ implies that $X_{0}$ and $X_{1}$ are closed linear subspaces of $X$. Since $b$ is non-zero, we have $X_{0} \neq X$ and $X_{1} \neq X$. From $b$-symmetry of $T$ it follows that $X_{0}$ and $X_{1}$ are both $T$-invariant. Hence $X_{0} \times X$ and $X \times X_{1}$ are $T \oplus T$-invariant proper closed subspaces of $X \times X$, they can not contain a cyclic vector for $T \oplus T$. Assume that $T \oplus T$ has a cyclic vector $(x, y) \in X \times X$. Then $x \notin X_{0}$ and $y \notin X_{1}$. Consider now a continuous linear operator $\Phi: X \times X \rightarrow Y$ defined by the formula

$$
\Phi(u, v)=b(x, v)-b(u, y) .
$$

Since $x \notin X_{0}$ and $y \notin X_{1}$, we have $\Phi \neq 0$. On the other hand, using $b$-symmetry of $T$, we have

$$
\Phi\left((T \oplus T)^{n}(x, y)\right)=b\left(x, T^{n} y\right)-b\left(T^{n} x, y\right)=0 .
$$

Thus the orbit of $(x, y)$ with respect to $T \oplus T$ lies in the proper closed linear subspace $\operatorname{ker} \Phi$ of $X$, which contradicts cyclicity of $(x, y)$ for $T \oplus T$.

Assume now that $b$ is non-symmetric. Then $c(x, y)=b(x, y)-b(y, x)$ is a non-zero separately continuous bilinear map from $X \times X$ to $Y$. Moreover, $T$ is $c$-symmetric. Assume that $x$ is a cyclic vector for $T^{2}$. Then $x$ can not lie in the right kernel of $c$, which is a proper closed $T$-invariant subspace of $X$. Hence the operator $\Psi \in L(X, Y), \Psi(u)=c(u, x)$ is nonzero. On the other hand, for any $n \in \mathbb{Z}_{+}$,

$$
\Psi\left(T^{2 n} x\right)=c\left(T^{2 n} x, x\right)=c\left(T^{n} x, T^{n} x\right)=b\left(T^{n} x, T^{n} x\right)-b\left(T^{n} x, T^{n} x\right)=0 .
$$

Hence the orbit of $x$ with respect to $T^{2}$ lies in the proper closed linear subspace $\operatorname{ker} \Psi$ of $X$, which contradicts cyclicity of $x$ for $T^{2}$.

This looks like a proper place to reproduce the following question of Grivaux.

Problem 13.15 Let $X$ be a Banach space and $T \in L(X)$ be such that $T$ $\oplus T$ is cyclic. Does it follow that $T^{2}$ is cyclic?

As a straightforward toy illustration of the above proposition one can consider the following fact. Let $(\Omega, \mathcal{F}, \mu)$ be a measure space, $g \in L_{\infty}(\mu), 0<\mathrm{p}<\infty$ and $T \in L\left(L_{p}(\mu)\right.$ be the operator of multiplication by $g: T f=f g$ for $f \in L_{p}(\mu)$. Then $T \oplus T$ is non-cyclic. Indeed, consider the continuous bilinear map $b: L_{p}(\mu) \times L_{p}(\mu) \rightarrow L_{p 2}(\mu)$, $b(f, h)=f h$. Clearly $b$ is non-zero and $T$ is $b$-symmetric. By Proposition 13.14, $T \oplus T$ is non-cyclic. The above mentioned result of Bayart and Matheron can now be formulated in the following way.

Theorem BM Let $X$ be a separable infinite dimensional Banach space with an unconditional Schauder basis such that the forward shift operator associated with this basis is bounded. Then there exists a hypercyclic $T \in L(X)$ and a non-zero continuous bilinear form $b: X \times X \rightarrow \mathbb{K}$ such that $T$ is $b$-symmetric. In particular, $T \oplus T$ is non-cyclic.

The form $b$ in the above theorem must be symmetric. Indeed, otherwise, by Proposition 13.14, $T^{2}$ is non-cyclic, which contradicts hypercyclicity of $T$ according to the Ansari theorem [1] on hypercyclicity of powers of hypercyclic operators. An answer to the following question could help in better understanding of the phenomenon of hypercyclic operators not satisfying the Hypercyclicity Criterion.

Problem 13.16 Let $T$ be a hypercyclic continuous linear operator on a Banach space $X$ such that $T \oplus T$ is non-hypercyclic. Does there exist a non-zero symmetric continuous bilinear form $b: X \times X \rightarrow \mathbb{K}$ such that $T \oplus T$ is $b$-symmetric?

It is worth noting that non-existence of such a form $b$ is equivalent to the density of the range of the operator $I \otimes T-T \otimes I$ acting on the projective tensor product $X \widehat{\otimes}_{\pi} X$.

Another observation concerning Theorem BM is that operators constructed in reference [50] have huge spectrum. Namely, their spectrum contains a disk centered at 0 of radius $>1$. On the other hand, we know (see Theorems 1.19 and 1.21) that any separable infinite dimensional complex Banach space supports plenty of hypercyclic operators with the spectrum being the singleton $\{1\}$. This leads to the following question.

Problem 13.17 Let $T$ be a hypercyclic continuous linear operator on a complex Banach space $X$ such that $\sigma(T)=\{1\}$. Is $T \oplus$ Thypercyclic?

It is worth noting that an affirmative answer to the above question would take care of Problem 13.7. Indeed, the spectrum of any hypercyclic operator on a hereditarily indecomposable complex Banach space [51] is a singleton $\{z\}$ with $z \in \mathbb{T}$.

\section{n-supercyclic operators}

Recently Feldman [52] has introduced the notion of an $n$-supercyclic operator for $n \in \mathbb{N}$. A bounded linear operator $T$ on a Banach space $X$ is called $n$-supercyclic for $n \in \mathbb{N}$ if there exists an $n$-dimensional linear subspace $L$ of $X$ such that its orbit $\{T x: n \in, x \in L\}$ is dense in $X$. Such a space $L$ is called an $n$ - supercyclic subspace for $T$. Clearly,1supercyclicity coincides with the usual supercyclicity. In reference [52], for any $n \in \mathbb{N}, n \geq 2$, a bounded linear operator $T$ on $\ell_{2}$ is constructed, which is $n$-supercyclic and not $(n-1)$-supercyclic. The construction is based on the observation that if $T_{k}$ for $1 \leq k \leq n$ are bounded linear operators on Banach spaces $X_{k}$ all satisfying the Supercyclicity Criterion with the same sequence $\left\{n_{k}\right\}$, then the direct sum $T_{1} \oplus \ldots \oplus T_{n}$ is $n$ -supercyclic. This observation leads to the natural question whether the direct sum of $n$ supercyclic operators should be $n$-supercyclic. The following proposition provides a negative answer to this question.

Proposition 13.18 There exists a hypercyclic operator $T \in L\left(\ell_{2}\right)$ such that $T \oplus T$ is not $n$-supercyclic for any $n \in \mathbb{N}$.

The above proposition follows immediately from Theorem BM and the next proposition, which implies that for the operator $T$ from Theorem BM, $T \bigoplus T$ is not $n$-supercyclic for any $n \in \mathbb{N}$.

Proposition 13.19 Let $X$ be an infinite dimensional topological vector space, $b: X \times X \rightarrow \mathbb{K}$ be non-zero, separately continuous and bilinear and $T \in L(X)$ be a b-symmetric operator with no non-trivial closed invariant subspaces of finite codimension. Then for any finite dimensional linear subspace $L$ of $X \times X$, the set $\{(p(T) x, p(T) y): p \in \mathcal{P},(x, y) \in L\}$ is nowhere dense in $X \times X$. 
In order to prove Proposition 13.19, we need the following lemma.

Lemma 13.20 Let $m \in \mathbb{N}, L$ and $X$ be topological vector spaces, such that $\operatorname{dim} L=k \leq m$ and $B: L \times X \rightarrow \mathbb{K}^{m}$ be a continuous bilinear map such that for each non-zero $n \in L$, the linear map $B(u, \cdot)$ is surjective. Then the set $A=\{x \in X: B(u, x)=0$ forsomenon-zero $u \in L\}$ is closed and nowhere dense in $X$.

Proof. First, observe that it is enough to prove the required statement in the case, when $X$ is finite dimensional. Indeed, let $e_{1}, \ldots$, $e_{k}$ be a basis of $L$ and $X_{1}=\bigcap_{j=1}^{k} k e r B\left(e_{j}, \cdot\right)$. Then $X_{1}$ is a closed linear subspace such that $B(u, x)=0$ for any $(u, x) \in L \times X_{1}$. Moreover, $X_{1}$ has codimension at most $\mathrm{km}$ in $X$. Thus we can pick a finite dimensional subspace $Y$ of $X$ such that $X=X_{1} \oplus Y$. Since $B(u, x)=0$ for any $(u, x) \in L \times X_{1}$, we have that for each $u \in L \backslash\{0\}$, the restriction of $B(u, \cdot)$ to $Y$ is onto. It is also clear that $A=\pi^{-1}\left(A_{0}\right)$, where $A_{0}=\{x \in Y: B(u, x)=0$ for some non-zero $u \in L\}$ and $\pi$ is the projection in $X$ onto $Y$ along $X_{1}$. Since $\pi$ is continuous and open, $A$ is closed and nowhere dense in $X$ if and only if $A_{0}$ is closed and nowhere dense in $Y$, which is finite dimensional.

Thus without loss of generality, we can assume that $X$ is finite dimensional. Consider the unit sphere $S$ in $L$ with respect to some Hilbert space norm on $L$. For each $u \in S, B(u, \cdot)$ is onto and we can pick an $m$-dimensional subspace $Z_{u}$ of $X$ such that the restriction of $B(u, \cdot)$ to $Z_{u}$ is invertible. Clearly, the set $V_{u}$ of those $v \in S$ for which the restriction of $B(u, \cdot)$ to $Z_{u}$ is invertible is open in $S$ and contains $u$. Thus we can pick a neighborhood $W_{u}$ of $u$ such that $B(v, \cdot)$ is onto for each $v \in \overline{W_{u}}$. Since $u \in W_{u}$, the family $\left\{W_{u}: u \in S\right\}$ is an open cover of the compact space $S$ and therefore we can choose $u_{1}, \ldots, u_{r} \in S$ such that

$$
\begin{aligned}
& S=\bigcup_{j=1}^{r} w_{u_{j}} \text {. Then } \\
& \qquad A=\bigcup_{j=1}^{r} A_{j} \text {, where } A_{j}=\left\{x \in X: B(u, x)=0 \text { for somenon - zero } u \in \overline{W_{u_{j}}}\right\} .
\end{aligned}
$$

It suffices to show that each $A_{j}$ is closed and nowhere dense. Let $1 \leq j \leq r$. Closeness of $A_{j}$ is rather easy. Indeed, let $\left\{x_{n}\right\}_{n \in \mathbb{Z}_{+}}$be a sequence of elements of $A_{j}$ converging to $x \in X$. Since $x_{n} \in A_{j}$, we can pick $w_{n} \in \overline{W_{u_{j}}}$ such that $B\left(w_{n}, x_{n}\right)=0$. Since $\overline{W_{u_{j}}}$ is compact, we, passing to a subsequence, if necessary, can assume that $w_{n} \rightarrow w \in \overline{W_{u_{j}}}$. Since $B$ is continuous, we have $0=B\left(w_{n}, x_{n}\right) \rightarrow B(w, x)$. Thus $B(w, x)=0$ and $x \in A_{j}$. That is, $A_{j}$ is closed. It remains to show that it is nowhere dense. Pick a linear subspace $Y_{j}$ of $X$ such that $Z_{u_{j}} \oplus Y_{j}=X$. For each $u \in V_{u_{j}}$ let $T_{u}$ be the restriction of $B(u, \cdot)$ to $Z_{u}$ and $S_{u}$ be the restriction of $B(u, \cdot)$ to $Y_{j}$. Let $z \in Z_{u_{j}}$ and $y \in Y_{j}$. Then $z+y \in A_{j}$ if and only if there exists $u \in \overline{W_{u_{j}}}$ such that $T_{u} z+S_{u} y=0$. Since $T_{u}$ is invertible, the latter is equivalent to $z=-T_{u}^{-1} S_{u} y$. Thus $A_{j}=F\left(Y_{j} \times \overline{W_{u_{j}}}\right)$, where $F: Y_{j} \times V_{u_{j}} \rightarrow X$, $F(y, u)=y-T_{u}^{-1} S_{u} y$. Let $n=\operatorname{dim} X$. Then $\operatorname{dim} Y_{j}=n-m$. Hence $Y_{j} \times V_{u_{j}}$ is a manifold of dimension $\alpha(n-m+k)-1$, where $\alpha=1$ if $\mathbb{K}=\mathbb{R}$ and $\alpha=1$ if $\mathbb{K}=\mathbb{C}$. It is clear that $F$ is smooth and therefor is Lipschitzian on any compact set. Since a Lipschitzian map does not increase the Hausdorff dimension, we see that $A_{j}=F\left(Y_{j} \times \overline{W_{u_{j}}}\right)$ is a countable union of compact sets of Hausdorff dimension at most $\alpha(n-m+k)-1<\alpha n$. Since $X \simeq \mathbb{K}^{n}$, any compact subset of $X$ of Hausdorff dimension $<\alpha n$ is nowhere dense. Thus $A_{j}$ is a Baire first category set. Since $A_{j}$ is closed, it is nowhere dense.

Proof of Proposition 13.9. First, we consider the case of nondegenerate $b$. That is, we assume that both the left and the right kernels $X_{0}$ and $X_{1}$ of $b$ defined by (13.2) are trivial. For each $k \in \mathbb{Z}_{+}$ and $(x, y) \in X \times X$, consider the linear functional $\Phi_{k}(x, y) \in(X \times X)^{\prime}$ defined by the formula

$$
\Phi_{k}(x, y)(u, v)=b\left(T^{k} x, v\right)-b\left(u, T^{k} y\right) .
$$

First, we shall check that for any $(x, y) \neq(0,0)$, the functionals $\Phi_{k}(x, y)$ for $k \in \mathbb{Z}_{+}$are linearly independent. Assume the contrary. Then there exists a non-zero polynomial $p$ such that $b(p(T) x, v)=b(u, p(T) y)$ for any $u, v \in X$. Since the left-hand side of the last equality does not depend on $u$ and the right-hand side does not depend on $v$, they both do not depend on both $u$ and $v$. Hence $b(p(T) x, v)=b(u, p(T) y)=0$ for any $u, v$ $\in X$. Since $T$ is $b$-symmetric, we have $b(x, p(T) v)=b(p(T) u, y)=0$ for any $u, v \in X$. Hence $p\left(T^{\prime}\right) \varphi=p\left(T^{\prime}\right) \psi=0$, where $\varphi, \psi \in X^{\prime}, \varphi(v)=b(x, v)$, $\psi(u)=b(u, y)$. Since $T$ has no non-trivial closed invariant subspaces of finite codimension, $T^{\prime}$ has no non-trivial finite dimensional invariant subspaces. By Lemma 7.2, $p\left(T^{\prime}\right)$ is injective. Hence $\varphi=\psi=0$. Since $b$ is non-degenerate, we then have $x=y=0$, which contradicts with the assumption $(x, y) \neq(0,0)$. Thus the functionals $\Phi_{k}(x, y)$ for $k \in \mathbb{Z}_{+}$are linearly independent for each $(x, y) \neq(0,0)$.

Let $L$ be a finite dimensional linear subspace of $X \times X$, $\operatorname{dim} L=k \in \mathbb{N}$ and $m \in \mathbb{N}, m>n$. Consider the bilinear map

$$
B: L \times(X \times X) \rightarrow \mathbb{K}^{m}, \quad B((x, y),(u, v))=\left\{\Phi_{j}(x, y)(u, v)\right\}_{j=0}^{m-1} .
$$

Since for any non-zero $(x, y) \in L$, the functionals $\Phi_{0}(x, y), \ldots, \Phi_{m-1}(x, y)$ are linearly independent, we see that the linear map $B((x, y), \cdot)$ is onto. By Lemma 13.20 , the set

$A=\{(u, v) \in X \times X$ : there is non - zero $(\mathrm{x}, \mathrm{y}) \in \mathrm{L}$ such that $\mathrm{B}((\mathrm{x}, \mathrm{y}),(\mathrm{u}, \mathrm{v}))=0\}$ is closed and nowhere dense in $X \times X$. Let now $(0,0) \neq(u, v) \in p(T \oplus T)(L)$ for some $p \in \mathcal{P}$. Then $(u, v)=(p(T) x, p(T) y)$ for some $(x, y) \in L \backslash\{(0,0)\}$. Then $\Phi_{j}(x, y)(u, v)=\left(b\left(T^{j} x, p(T) y\right)-b\left(p(T) x, T^{j} y\right)\right)=0$ for any $j \in \mathbb{Z}_{+}$since $T$ is $b$-symmetric. It follows that $(u, v) \in A$. That is, the nowhere dense set $A$ contains the set $\{(p(T) x, p(T) y): p \in \mathcal{P},(x, y) \in L\}$. Thus the latter set is nowhere dense.

It remains to reduce the general case to the case of nondegenerate $b$. Since $b$ is non-zero, at least one of the bilinear forms $b_{0}(x, y)=b(x, y)+b(y, x) \quad$ or $\quad b_{1}(x, y)=b(x, y)-b(y, x)$ is non-zero. Clearly $T$ is symmetric with respect to both $b_{0}$ and $b_{1}$. Thus replacing $b$ by either $b_{0}$ or $b_{1}$, if necessary, we can assume that $b$ is symmetric or antisymmetric. Then left and right kernels $X_{0}$ and $X_{1}$ of $b$ defined by (13.2) coincide. Since $T$ is $b$-symmetric, $X_{0}$ is $T$-invariant. Let $T_{0} \in L\left(X / X_{0}\right)$ be defined as $T_{0}\left(x+X_{0}\right)=T x+X_{0}$ and $\beta$ be the bilinear form on $X / X_{0}$ defined by the formula $\beta\left(x+X_{0}, y+X_{0}\right)=b(x, y)$. Since $X_{0}$ is the right and the left kernel of $b$ and is $T$-invariant, the operator $T_{0}$ and the form $\beta$ are well-defined and $\beta$ is non-degenerate. Moreover, it is easy to see that $T_{0}$ is $\beta$-symmetric and has no non-trivial closed invariant subspaces of finite codimension. Assume now that $L$ a finite dimensional linear 
subspace $L$ of $X \times X, A=\{(p(T) x, p(T) y): p \in \mathcal{P},(x, y) \in L\}$ and $B=\left\{\left(p\left(T_{0}\right)\left(x+X_{0}\right), p\left(T_{0}\right)\left(y+X_{0}\right)\right): p \in \mathcal{P},(x, y) \in L\right\} \cdot$ According to the first part of the proof, $B$ is nowhere dense in $X / X_{0}$. Since $A=\pi^{-1}(B)$, where $\pi(x)=x+X_{0}$ is the canonical map from $X$ onto $X / X_{0}$, we see that $A$ is nowhere dense in $X$. [53].

The following question is raised by Bourdon, Feldman and Shapiro

Problem 13.21 Let $X$ be a complex Banach space, $n \in \mathbb{N}$ and $T \in$ $L(X)$ be such that $T$ is $n$-supercyclic and $\sigma_{p}\left(T^{\prime}\right)=\varnothing$. Is $T$ cyclic?

It is worth noting that the only known examples of $n$-supercyclic operators $T$ with $\sigma_{p}\left(T^{\prime}\right)=\varnothing$ are the mentioned direct sums of operators satisfying the Supercyclicity Criterion with the same sequence $\left\{n_{k}\right\}$. Such direct sums are all cyclic [54].

Feldman in reference [52] has also introduced the concept of an $\infty$-supercyclic operator. A bounded linear operator $T$ on a Banach space $X$ is called $\infty$-supercyclic if there exists a linear subspace $L$ of $X$ such that its orbit $\left\{T^{n} x: n \in \mathbb{Z}_{+}, t \in L\right\}$ is dense in $X$, the space $T^{n}(L)$ is not dense in $X$ for any $n \in \mathbb{Z}$ and $L$ contains no non-zero invariant subspace of $T$. Gallardo and Motes-Rodriguez [55], answering a question of Salas, demonstrated that the Volterra operator

$$
V: L_{2}[0,1] \rightarrow L_{2}[0,1], \quad V f(x)=\int_{0}^{x} f(t) d t
$$

is not supercyclic. In [56] it is shown that $V$ is not $n$-supercyclic for any $n \in \mathbb{N}$. However, it turns out that $V$ is $\infty$-supercyclic.

Proposition 13.22 The Volterra operator is $\infty$-supercyclic.

Proof. For any non-zero $h \in L_{2}[0,1]$ we denote by $L_{h}$ the orthocomplement of $h: L_{h}=\left\{f \in L_{2}[0,1]:\langle f, h\rangle=0\right\}$. It is straightforward to see that for any $h \in L_{2}[0,1]$,

$$
\overline{V\left(L_{V^{*} h}\right)}=L_{h}, \text { where } V^{*} f(x)=\int_{x}^{1} f(t) d t
$$

is the adjoint of $V$. Consider the space $\varepsilon=\left\{f \in C^{\infty}[0,1]: f^{(j)}(1)=0\right.$ for any $\left.j \in \mathbb{Z}_{+}\right\}$. If $h$ is non-zero element of $\varepsilon$, then according to the above display, $V^{*} h^{\prime}=-h$. Thus by (13.3)

$$
\overline{V^{n}\left(L_{h}\right)}=L_{h^{(n)}} \text { for any non-zero } h \in \varepsilon \text { and any } n \in \mathbb{Z}_{+} \text {. }
$$

Consider now the following specific $h \in \varepsilon: h(1)=0$ and $h(x)=e^{(x-1)^{-1}}$ for $0 \leq x<1$. First, we shall show that $L_{h}$ does not contain any non-zero invariant subspace of $V$. Assume the contrary. Then there exists non-zero $f \in L_{2}[0,1]$ such that $V^{n} f \in L_{h}$ for each $n \in$

$\mathbb{Z}_{+}$. That is, $0=\left\langle V^{n} f, h\right\rangle=\left\langle f, V^{*} n h\right\rangle$. Hence $h$ is not a cyclic vector for $V^{*}$. On the other hand, it is well-known that $g \in L_{2}[0,1]$ is non-cyclic for $V^{*}$ if and only if there is $q \in(0,1)$ such that $g$ vanishes on $[q, 1]$. Thus $h$ vanishes on a neighborhood of 1 , which is obviously not the case. Hence $L_{h}$ does not contain any non-zero invariant subspace of $V$. According to (13.4), $\overline{V^{n}\left(L_{h}\right)}$ is a closed hyperplane in $L_{2}[0,1]$ and therefore $V^{n}\left(L_{h}\right)$ is not dense in $L_{2}[0,1]$ for each $n \in \mathbb{Z}_{+}$. Now, in order to show that $V$ is $\infty$-supercyclic it suffices to verify that $A=\bigcup_{n=0}^{\infty} V^{n}\left(L_{h}\right)$ is dense in $L_{2}[0,1]$. By (13.4), $A=\bigcup_{h^{\prime}}^{\infty} L_{h}$. It is easy to see that for any $f \in$ $L_{2}[0,1]$ and non-zero $g \in L_{2}[0,1]$, the distance from $f$ to $L_{g}$ is given by the formula $\operatorname{dist}\left(f, L_{g}\right)=\|g\|^{-1}|\langle f, g\rangle|$. Let $q \in(0,1)$ and $f \in L_{2}[0,1]$ be such that $f$ vanishes on $[q, 1]$. Then

$$
\operatorname{dist}\left(f, L_{h^{(n)}}\right)=\frac{\left|\left\langle f, h^{(n)}\right\rangle\right|}{\left\|h^{(n)}\right\|} \leq\|f\| \frac{\left\|h^{(n)}\right\|_{L_{2}[0, q]}}{\left\|h^{(n)}\right\|_{L_{2}[0,1]}} .
$$

On the other hand, analyticity of $h$ on $[0, q]$ and easy lower estimates if $\left\|f^{(n)}\right\|_{L_{2}[0,1]}$ imply that

$$
\varlimsup_{n \rightarrow \infty}\left(n !\left\|h^{(n)}\right\|_{L_{2}[0, q]}\right)^{1 / n}<\infty \text { and } \lim _{n \rightarrow \infty}\left(n !\left\|h^{(n)}\right\|_{L_{2}[0, q]}\right)^{1 / n}=\infty .
$$

From the last two displays it follows that $\operatorname{dist}\left(f, L_{h^{(n)}}\right) \rightarrow 0$ as $n \rightarrow \infty$. Hence $\bar{A}$ contains the space of all functions vanishing on a neighborhood of 1 . Since the latter space is dense in $L^{2}[0,1], A$ is dense in $L^{2}[0,1]$, which completes the proof.

\section{$\mathbb{R}$-cyclicity and supercyclicity}

Let $T$ be a continuous linear operator on a separable complex topological vector space $X$. We say that $T$ is $\mathbb{R}$ - cyclic if there exists $x \in$ $X$ such that the linear span of the orbit $\left\{T^{n} x: n \in \mathbb{Z}_{+}\right\}$in $X$ considered as a linear space over $\mathbb{R}$ is dense in $X$. Similarly, $T$ is called $\mathbb{R}$ - supercyclic if there is $x \in X$ such that $\left\{t T^{n} x: n \in \mathbb{Z}, t \in \mathbb{R}\right\}$ is dense in $X$ and $T$ is called $\mathbb{R}^{+}$- supercyclic if there is $x \in X$ such that $\left\{t T^{n} x: n \in \mathbb{Z}_{+}, t>0\right\}$ is dense in $X$. Clearly any $\mathbb{R}^{+}$-supercyclic operator is $\mathbb{R}$-supercyclic and any $\mathbb{R}$-supercyclic operator is $\mathbb{R}$-cyclic. The following theorem by LeónSaavedra and Müller is proved in reference [57]. It is proved in the case when $X$ is a Banach space, but exactly the same proof works in general.

Theorem LM Let $T$ be a continuous linear operator on a complex locally convex space $X$ with $\sigma_{p}\left(T^{\prime}\right)=\varnothing$. Then $T$ is supercyclic if and only if $T$ is $\mathbb{R}^{+}$-supercyclic.

As we have shown, there are bilateral weighted shifts $T_{w}$ on $\ell_{2}(\mathbb{Z})$ with the weight sequence $w$ converging to zero arbitrarily fast and such that $I+T_{w}$ and $I+T^{\prime}{ }_{w}$ are both hypercyclic. This happens because we allow $w$ to behave irregularly while still satisfying the condition $\left|w_{n}\right| \leq a_{|n|}$ for every $n \in \mathbb{Z}$ with $a$ being any sequence of positive numbers. The following proposition shows that hypercyclicity of $I+T_{w}$ is incompatible with the symmetry of the weight sequence. Recall that a continuous linear operator on a Banach space $X$ is called weakly supercyclic if it is supercyclic on $X$ with weak topology.

Proposition 13.23 Let $w \in \ell_{\infty}(\mathbb{Z})$ be a weight such that $\left|w_{n}\right|=\left|w_{-n}\right|$ for any $n \in \mathbb{Z}$, and let $p$ be a polynomial with real coefficients. Then the operator $p\left(T_{w}\right)$ acting on complex $\ell_{2}(\mathbb{Z})$ is not $\mathbb{R}$-cyclic. In particular, by Theorem $\mathrm{LM}, p\left(T_{w}\right)$ is not weakly supercyclic.

Corollary 13.24 Let $w \in \ell_{\infty}(\mathbb{Z})$ with $\left|w_{n}\right|=\left|w_{-n}\right|$ for any $n \in \mathbb{Z}$. Then the operator $I+T_{w}$ acting on $\ell_{2}(\mathbb{Z})$ is not weakly supercyclic.

Proof of Proposition 13.23. First, note that if $w, w^{\prime} \in \ell_{\infty}(\mathbb{Z})$ satisfy $\left|w_{n}\right|=\left|w_{n}^{\prime}\right|$ for any $n \in \mathbb{Z}$, then $T_{w}$ and $T_{w^{\prime}}$ are isometrically similar with a diagonal unitary operator implementing the similarity. Thus we can, without loss of generality, assume that $w_{n} \in \mathbb{R}$ for each $n \in \mathbb{Z}$. Then the operators $T_{w}$ and $S=p\left(T_{w}\right)$ have real matrix coefficients with respect to the canonical basis. Let $H$ be the $\mathbb{R}$-subspace of $\ell_{2}(\mathbb{Z})$ consisting of the sequences with real entries. Then $T_{w}(H) \subseteq H, S(H) \subseteq H$, $T_{w}(i H)=i H$ and $S(i H) \subseteq i H$. Let $T_{0}$ be the restriction of $T_{w}$ to $H$ and 
$S_{0}$ the restriction of $S$ to $H$ considered as $\mathbb{R}$-linear operators. Since $T_{0}$ and $S_{0}$ are similar to the restrictions of $T_{w}$ and $S$ to $i H$, we see that $T_{w}$ and $S$, considered as $\mathbb{R}$-linear operators, are similar to $T_{0} \oplus T_{0}$ and $S_{0}$ $\bigoplus S_{0}$. Now the symmetry of the weight sequence $w$ implies that $T_{0}$ is isometrically similar to $T_{0}^{\prime}$. Indeed, $T_{0}^{\prime}=U T_{0} U^{-1}$, where $U e_{n}=e_{1-n}$ for $n$ $\in \mathbb{Z}$. Then $S_{0}=p\left(T_{0}\right)$ is similar to $S_{0}^{\prime}=p\left(T^{\prime}\right)$. Thus $S$ considered as an $\mathbb{R}$ -linear operator is similar to $S_{0} \oplus S_{0}^{\prime}$. The last operator is non-cyclic. Indeed, if $x \oplus y$ is a non-zero vector in $H \oplus H$, then the orbit of $x \oplus y$ with respect to $S_{0} \oplus S_{0}^{\prime}$ is orthogonal to the non-zero vector $y \oplus(-x)$ $\in H \oplus H$. Thus $S$ is not $\mathbb{R}$-cyclic.

Since $\ell_{p}(\mathbb{Z})$ for $1 \leq p \leq 2$ is contained in $\ell_{2}(\mathbb{Z})$ and carries a stronger topology than the one inherited from $\ell_{2}(\mathbb{Z})$, Proposition 13.23 remains true if we replace $\ell_{2}(\mathbb{Z})$ by $\ell_{p}(\mathbb{Z})$ with $1 \leq p \leq 2$. On the other hand, the unweighted shift on $\ell_{p}(\mathbb{Z})$ with $2<p<\infty$ is weakly supercyclic [37] and therefore the statement of Proposition 13.23 becomes false if we replace $\ell_{2}(\mathbb{Z})$ by $\ell_{p}(\mathbb{Z})$ with $p>2$. At this point it is interesting to remind that, according to Theorem $\mathrm{B}$, norm hypercyclicity and supercyclicity of a bilateral weighted shift on $\ell_{p}(\mathbb{Z})$ do not depend on $p$. This leads naturally to the following question.

Problem 13.25 Characterize hypercyclicity and supercyclicity of the operators of the form $I+T$, where $T$ is a bilateral weighted shift on $\ell_{p}(\mathbb{Z})$. In particular, does hypercyclicity or supercyclicity of these operators depend on the choice of $P .1 \leq p<+\infty$ ?

\section{References}

1. Ansari SI (1995) Hypercyclic and cyclic vectors. J Funct Anal 128: 374-383.

2. Bés J, Peris A (1999) Hereditarily hypercyclic operators. J Funct Anal 167: 94112.

3. Grivaux S (1993) Hypercyclic operators, mixing operators and the bounded steps problem. J Operator Theory 54: 147-168.

4. Grosse-Erdmann KG (1999) Universal families and hypercyclic operator. Bull Amer Math Soc 36: 345-381.

5. Grosse-Erdmann KG (2003) Recent developments in hypercyclicity, RACSAM Rev. R. Acad. Cienc. Exactas Fis. Nat Ser A Mat 97: 273-286.

6. Montes-Rodrguez A, Salas H (2001) Supercyclic subspaces: spectral theory and weighted shifts. Adv Math 163 74-134.

7. Wengenroth $J(2003)$ Hypercyclic operators on non-locally convex spaces. Proc Amer Math Soc 131: 1759-1761.

8. Herzog G (1992) On linear operators having supercyclic vectors. Studia Math 103: 295-298.

9. Ansari SI (1997) Existence of hypercyclic operators on topological vector spaces. J Funct Anal 148: 384-390.

10. Bernal-González L (1999) On hypercyclic operators on Banach spaces. Proc Amer Math Soc 127: 1003-1010.

11. Bonet J, Peris A (1998) Hypercyclic operators on non-normable Fréchet spaces. J Funct Anal 159: 587-595.

12. Salas H (1995) Hypercyclic weighted shifts. Trans Amer Math Soc 347: 9931004.

13. Bermúdez T, Bonilla A, Martinón A (2002) On the existence of chaotic and hypercyclic semigroups on Banach spaces. Proc Amer Math Soc 131: 24352441.

14. Bernal-González L, Grosse-Erdmann KG (2007) Existence and nonexistence of hypercyclic semigroups. Proc Amer Math Soc 135: 755-766.

15. Bermúdez T, Bonilla A, Conejero J, Peris A (2005) Hypercyclic, topologically mixing and chaotic semigroups on Banach spaces. Studia Math 170: 57-75.

16. Conejero $J(2007)$ On the existence of transitive and topologically mixing semigroups. Bull Belg Math Soc 14: 463-471.

17. Schäfer H (1966) Topological Vector Spaces. MacMillan, New York.
18. Herzog G, Lemmert R (1993) Über Endomorphismen mit dichten Bahnen. Math Z 213: 473-477.

19. Bés J, Conejero JA (2006) Hypercyclic subspaces in omega. J Math Anal App 316: 16-23.

20. Petersson H (2007) Hypercyclicity in omega. Proc Amer Math Soc 135: 11451149.

21. Chan K, Sanders R (2004) A weakly hypercyclic operator that is not norm hypercyclic. J Operator Theory 52: 39-59.

22. Bonet J, Frerick L, Peris A, Wengenroth J (2005) Transitive and hypercyclic operators on locally convex spaces. Bull London Math So. 37: 254-264.

23. Salas H (1991) A hypercyclic operator whose adjoint is also hypercyclic. Proc Amer Math Soc 112:765-770.

24. Petersson $H$ (2005) Spaces that admit hypercyclic operators with hypercyclic adjoint. Proc Amer Math Soc 134: 1671-1676

25. Salas H (2007) Banach spaces with separable duals support dual hypercyclic operators. Glasg Math J 49: 281-290.

26. Lindenstrauss J, Tzafriri L (1997) Classical Banach spaces I. Sequence spaces, Springer, Berlin.

27. Godefroy G, Shapiro JH (1991) Operators with dense, invariant cyclic vector manifolds. J Funct Anal 98: 229-269.

28. Montes-Rodrguez A, Rodrguez-Martinez A and Shkarin S (2006) Volterra type operators: spectral theory and cyclicity. Universidad de Sevilla 184.

29. Bonet J, Pérez-Carreras P (1987) Barrelled locally convex spaces. NorthHolland Mathematics Studies, North-Holland Publishing Co., Amsterdam, Netherlands.

30. Hilden H, Wallen L (1973) Some cyclic and non-cyclic vectors of certain operators. Indiana Univ Math J 23: 557-565.

31. Salas H (1995) Supercyclicity and weighted shifts. Studia Math 135: 55-74.

32. Bayart, Grivaux S (2005) Hypercyclicity and unimodular point spectrum. J Funct Anal 226: 281-300.

33. Herrero D, Wang Z (1990) Compact perturbations of hypercyclic and supercyclic operators. Indiana Univ Math J 39: 819-829.

34. Grivaux S (2003) Hypercyclic operators with an infinite dimensional closed subspace of periodic points. Rev Mat Complut 16: 383-390.

35. Badea C, Grivaux S (2007) Unimodular eigenvalues, uniformly distributed sequences and linear dynamics. Adv Math 211: 766 - 793.

36. Oxtoby $\mathrm{J}(1980)$ Measure and category. A survey of the analogies between topological and measure spaces. Springer, New York.

37. Shkarin S (2007) Non-sequential weak supercyclicity and hypercyclicity. J Funct Anal 242: 37-77.

38. Shkarin S (2000) An infinite-dimensional pre-Hilbert space that is nonhomeomorphic to a closed hyperplane of it. Izv Math 63: 1263-1273.

39. Grivaux S (2003) Construction of operators with prescribed behaviour. Arch Math (Basel) 81: 291-299.

40. Valdivia M (1982) Topics in locally convex spaces. North Holland, Amsterdam.

41. Pol R (1984) An infinite dimensional pre-Hilbert space non-homeomorphic to its owm square. Proc Amer Math Soc 90: 450-454.

42. Van Mill J (1987) An infinite-dimensional pre-Hilbert space all bounded linea operators of which are simple. Colloq Math 54: 29-37.

43. Marciszewski W (1983) An infinite dimensional pre-Hilbert space without a continuous map on its own square. Bull Acad Polon Sci ser math 31: 393-396.

44. Shkarin S (2001) Three constructive examples of pre-Hilbert spaces nonisomorphic to their closed hyperplanes. Russ J Math Phys 8: 106-121.

45. Kalton N, Peck N, Roberts J (1984) An F-space sampler. London Mathematical Society Lecture Note Series 89, Cambridge University Press, Cambridge.

46. Kitai C (1982) Invariant closed sets for linear operators. Thesis, University of Toronto.

47. Gethner RM, Shapiro JH (1987) Universal vectors for operators on spaces of holomorphic functions. Proc Amer Math Soc 100: 281-288. 
48. Markushevich A (1965) Theory of functions of a complex variable I. American Mathematical Soc Mathematics 1138.

49. De La Rosa M, Read C (2009) A hypercyclic operator whose direct sum $\mathrm{T} \oplus \mathrm{T}$ is not hypercyclic. J Operator Theory 61: 369-380.

50. Bayart F, Matheron E (2007) Hypercyclic operators failing the hypercyclicity criterion on classical Banach spaces. J Funct Anal 250: 426-441

51. Gowers WT, Maurey B (1993) The unconditional basic sequence problem. J Amer Math Soc 6: 851-874.

52. Feldman N (2002) N-supercyclic operators. Studia Math 151: 141-159.
53. Bourdon P, Feldman N, Shapiro J (2004) Some properties of $N$-supercyclic operators. Studia Math 165: 135-157.

54. Shkarin S (2008) Universal elements for non-linear operators and their applications. J Math Anal Appl 348: 193-210

55. Gallardo-Gutiérrez EA, Montes-Rodrguez A (2004) The Volterra operator is not supercyclic. Integral Equations Operator Theory 50: 211-216.

56. Shkarin S (2006) Antisupercyclic operators and orbits of the Volterra operator. J Lond Math Soc 73: 506-528.

57. León-Saavedra F, Müller V (2004) Rotations of hypercyclic and supercyclic operators. Integral Equations Operator Theory 50: 385-391.
This article was originally published in a special issue, Algebra, Combinatorics and Dynamics handled by Editor. Dr. Natalia lyudu, Researcher School of Mathematics, The University of Edinburgh, UK 